Check for updates

Cite this: Mater. Adv., 2021, 2, 2811

Received 5th January 2021 Accepted 28th March 2021

DOI: $10.1039 / \mathrm{d} 1 \mathrm{ma} 00008 \mathrm{j}$

rsc.li/materials-advances

\section{Conquering the crystallinity conundrum: efforts to increase quality of covalent organic frameworks}

\author{
Laurens Bourda, (D) ab Chidharth Krishnaraj, (D) ${ }^{\mathrm{b}}$ Pascal Van Der Voort (D) ${ }^{\mathrm{b}}$ and \\ Kristof Van Hecke (iD *a
}

\begin{abstract}
Due to their high designability, covalent organic frameworks (COFs) are attractive candidates in many different applications. However, COF synthesis is still poorly understood and mainly trial and error based. In this review, we pave the way to a better understanding of COF chemistry and the synthesis of highquality COFs. To achieve this goal, the main challenge in COF synthesis, the crystallization problem, is explained. Additionally, mechanisms for boroxine and imine COF formation are proposed. Afterwards, several approaches to increase COF quality are discussed: addition of modulators, slowing down monomer addition, improvement of used catalysts, study of solubility effects and interfacial synthesis, application of exchange reactions and finally linker specific observations. The discussed techniques can be seen as a toolbox usable for the synthesis of high-quality COFs.
\end{abstract}

\section{Introduction}

The recent discovery of covalent organic frameworks (COFs) in $2005^{1}$ can be acknowledged as the "pièce de resistance" of almost a century of increasing control over the dynamic bond. In 1916, Lewis published "The atom and the molecule",' a crucial paper visualizing the bonding of atoms in molecules through the concept of covalent bond. Today, just over 100 years later, chemists are able to grow single crystals of extended 3D structures. $^{3}$ To reach this stage, several important milestones had to be crossed, ${ }^{4}$ of which the most important ones are presented in Fig. 1.

Staudinger's "Über Polymerisation", 5,6 established in 1920, can be regarded as the start of polymer science. Thereon, a new field studying these 1D macromolecules emerged, resulting in Staudinger being awarded the 1953 Nobel Prize in Chemistry. Meanwhile, with the first total synthesis (urea) already reported in $1828^{7}$ and the synthesis of mauveine ${ }^{8}$ and aspirin ${ }^{9}$ having revolutionized the dye and pharmaceutical industries, organic synthesis was becoming an ever growing field. ${ }^{10}$ In the second half of the 20th century, a high level of synthetic control had already been established, illustrated by the achievements of Robert Burns Woodward ${ }^{11}$ (Nobel Prize in Chemistry, 1965) who synthesized a whole array of compounds including quinine, respirine, chlorophyll and the famous vitamin $\mathrm{B} 12^{12}(1972$, with

\footnotetext{
${ }^{a}$ XStruct, Department of Chemistry, Ghent University, Krijgslaan 281, Building S3, 9000 Ghent, Belgium. E-mail: kristof.vanhecke@ugent.be

${ }^{b}$ COMOC, Center for Ordered Materials, Organometallics \& Catalysis, Department of Chemistry, Ghent University, Krijgslaan 281, Building S3, 9000 Ghent, Belgium. E-mail: pascal.vandervoort@ugent.be
}

Eschenmoser) which was the most complex natural occurring product synthesized in the lab at that time.

Thus, by the end of the 20th century organic synthesis had evolved to a mature science and (1D) macromolecules had been developed to a great extent and found industrial applications. But still, no extended organic 2D nor 3D structures were reported, a blind spot aptly illustrated by Roald Hoffmann in 1993 "Organic chemists are masterful at exercising control in zero dimensions. One subculture of organic chemists has learned to exercise control in one dimension. But in two or three dimensions, it's a synthetic wasteland. The methodology for exercising control so that one can make unstable but persistent extended structures on demand is nearly absent". ${ }^{13}$ Still, since the 1960's, great process had already been made in a field called "supramolecular chemistry", where quite sophisticated architectures could be built. Important breakthroughs were the synthesis of crown ethers ${ }^{14}$ and cryptands, ${ }^{15}$ by Pedersen and Lehn, respectively. Additionally, the first permanent porous materials were created using supramolecular chemistry and reported as shape-persistent cages in 1984 by Vögle ${ }^{16}$ and Cram. ${ }^{17}$ Pedersen, Lehn and Cram received the Nobel prize in Chemistry 1987 for their developments in supramolecular chemistry. This field is based on non-covalent interactions, which allows high reversibility and thermodynamic control with error-correction and self-assembly as highly attractive features. However, due to the associated weak, non-covalent bonds, modification of these structures proved difficult, as losses of structural integrity and alterations of the binding interactions are often observed as a consequence of functionalization. Therefore, one needed to be able to control the formation and error-correction of the stronger covalent bonds, which was realized with dynamic covalent 


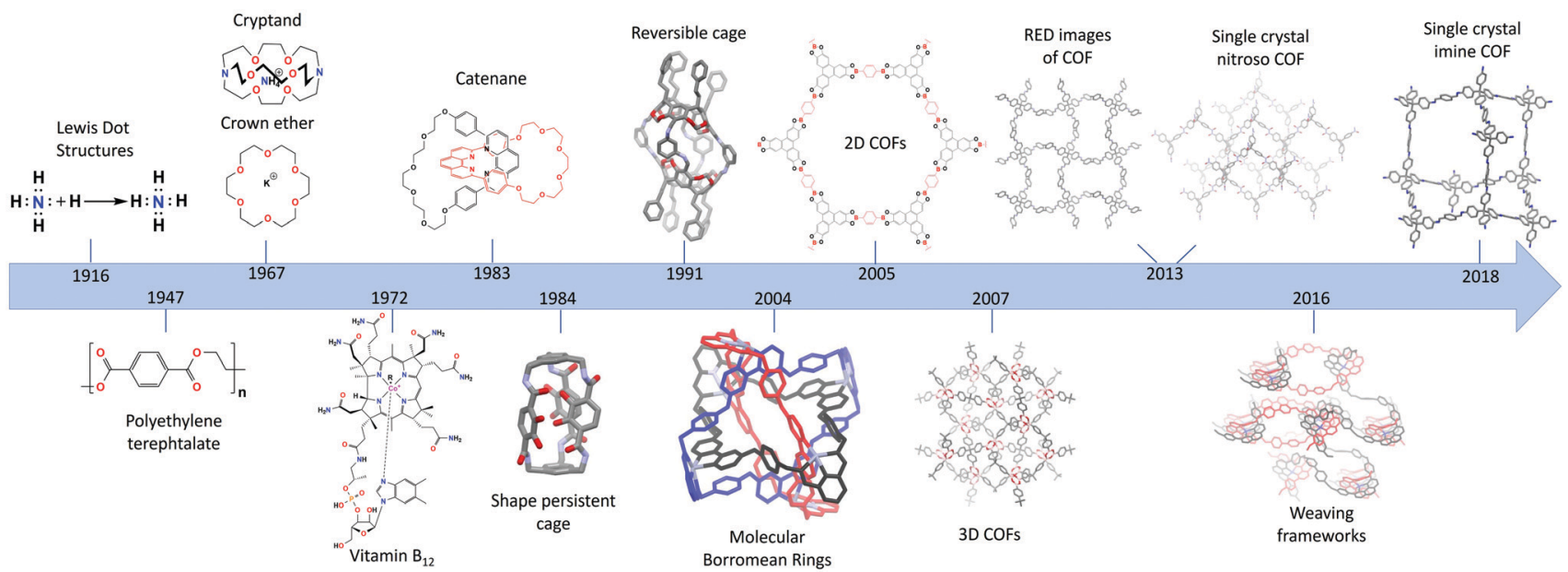

Fig. 1 From the covalent bond to single crystal COFs, an overview of milestones in COF history.

chemistry (DCC) and its kinetic reaction control. ${ }^{18}$ The field of mechanically interlocking molecules, with the first catenane, consisting of two interlocking rings, synthesized in 1983 by Sauvage $^{19}$ was crucial for the development of DCC. As no dynamic chemistry was used here, the yield was only $72 \%$, which would be disastrous for complicated structures containing far more rings. Since then, many different catenanes have been developed based on reversible covalent reactions with a large contribution from Sir. James F. Stoddart. His molecular borromean rings (2004) are an elegant example of the possibilities of this type of chemistry, as he could obtain three mechanically interlocking rings with a $100 \%$ yield. ${ }^{20}$ In 2016 Sauvage and Stoddart received the Nobel prize in Chemistry (together with Feringa) for their contributions in DCC and molecular machines. DCC was also used to create shape-persistent cages in high yields,${ }^{21}$ allowing the creation of a large variety of sizes and shapes of permanently porous cages. ${ }^{22}$

In $2005(2 \mathrm{D})^{1}$ and $2007(3 \mathrm{D})^{23}$ extended crystalline COFs could be synthesized using these reversible covalent linkages. As these frameworks are completely composed of strong covalent bonds they are expected to be highly stable and can be easily modified. However, some exceptions still exist, as will be discussed in Section 1.1.1. Furthermore, the combination of their intrinsic porosity and chemical robustness makes it possible to obtain materials with high permanent porosities. Over the years, the achievable crystallinity of COFs improved rapidly, which led to two major breakthroughs in 2013 when single crystal structures of COFs could be solved. Yaghi et al. succeeded in the synthesis of COF-320 with crystals up to $200 \mathrm{~nm}$ which could be solved using Rotation Electron Diffraction (RED) ${ }^{24}$ and Wuest et al. obtained crystals of nitroso polymer networks suitable for Single Crystal X-ray Diffraction (SXRD) analysis. ${ }^{25}$ An interesting subset are the class of woven COFs (wCOFs), firstly reported by the Yaghi group in $2016 .{ }^{26}$ These materials are made through the molecular weaving of distinct threads. While the removable "crossings" of those threads are composed of metal-coordination bonds, the threads are completely composed of strong covalent bonds, making these materials essentially COFs. Moreover, the single crystal structures of these materials could be solved using 3D-Electron Diffraction Tomography (3D-EDT). Finally, in 2018 , ultimate control was reached, with the synthesis of 3D COF crystals of up to $60 \mu \mathrm{m}$, linked by strong imine bonds. ${ }^{3}$

\subsection{Design of COFs}

One of the main advantages of COFs is their high designability. By carefully choosing functional groups and symmetry of the used building blocks, COFs with specific functionalities and framework structures can be obtained. ${ }^{27}$ In theory, linkers only require a rigid backbone allowing directional bonding and reactive end-groups able to trigger dynamic covalent reactions for making highly crystalline COFs. However, in practice this is not that convenient.

1.1.1 Linkage design. Since the inception of COFs in 2005, many different linkages have been developed, with a varying degree of reversibility and thus crystallinity (Fig. 2). Generally, higher reversibility means high crystallinity but lower stability of the obtained material as the linking bond is not sufficiently strong. To counter this problem, various post-modification ${ }^{28}$ and stabilization pathways ${ }^{29}$ have been studied. For example, the first COFs were reported using boroxine (COF-1), boronate ester (COF-5, COF-102, COF-103, COF-105, COF-108) and borosilicate linkages (COF-202) and the reversibility of the synthesis is ensured by carefully managing the amount of water in the system. ${ }^{1,23,30}$ This way, high crystallinity could be obtained but these materials are also quite susceptible to framework damage in the presence of water. The antipode of these $\mathrm{B}-\mathrm{O}$ linkages can be found in the cyclic $-\mathrm{C}=\mathrm{N}$ - linkage, which gives rise to extremely stable materials. ${ }^{31}$ However, even with harsh synthetic conditions $\left(400{ }^{\circ} \mathrm{C}\right.$, molten $\left.\mathrm{ZnCl}_{2}\right)$, the obtained crystallinity can be referred to as moderate at best. Since the first report of COF-300 in $2009,{ }^{32}$ the Schiff base imine linkage and its derivatives have arguably become the most extensively studied linkers in COF chemistry. This can be partially attributed to the high availability and affordability of a large variety of aldehyde and amine precursors along with the 


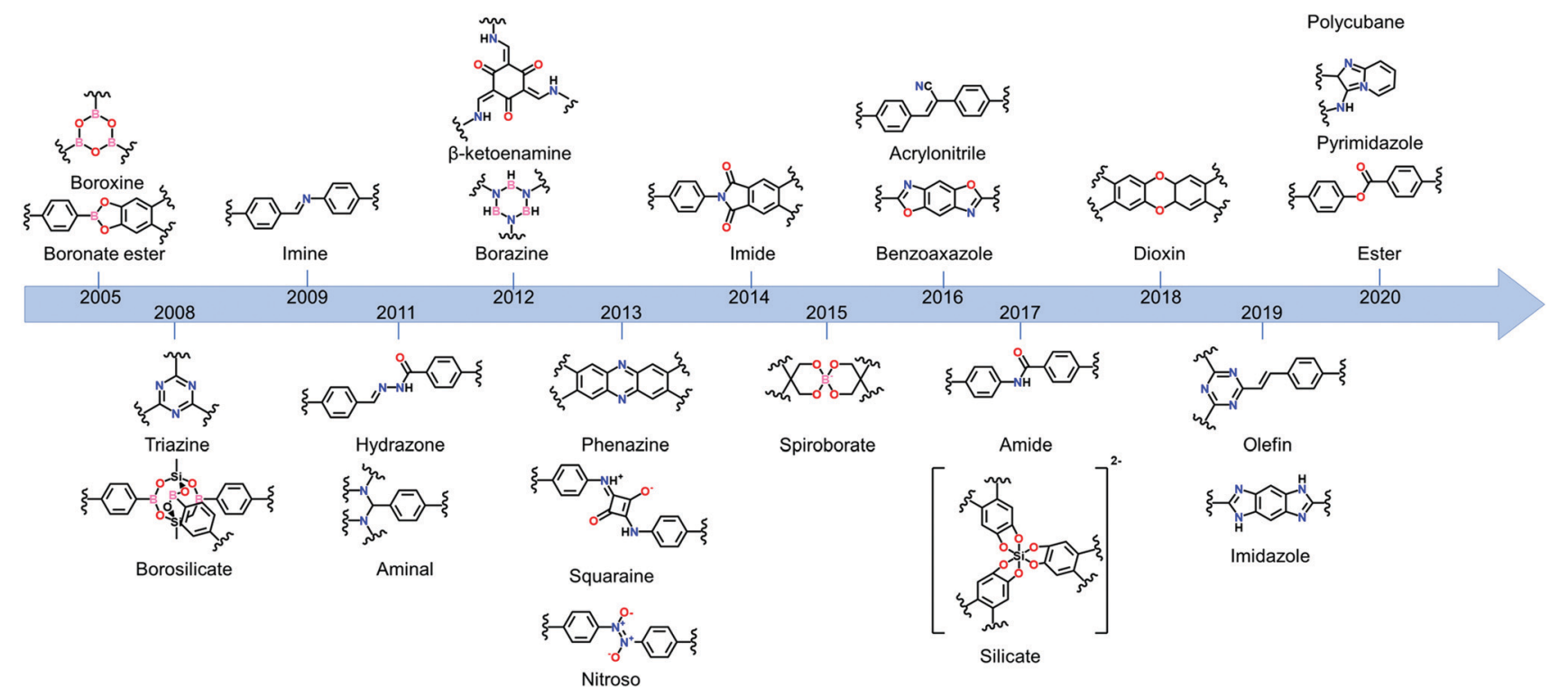

Fig. 2 Evolution of developed COF linkages

increased linkage stability in humid environments and neutral to basic $\mathrm{pH} .{ }^{33}$ Moreover, the employed Schiff base reactions are well studied and can easily be controlled by the addition of acid catalysts. Over the next years, Schiff base chemistry has also been used to make different related linkages like hydrazone, ${ }^{34}$ $\beta$-ketoenamine, ${ }^{35}$ phenazine, ${ }^{36}$ squaraine $^{37}$ and benzoxazole ${ }^{38}$ COFs. Particularly the $\beta$-ketoenamine linkage is interesting as it combines a highly reversible, imine condensation with a subsequent irreversible tautomerization to a highly stable $\beta$-ketoenamine COF. C-N bonds could also be used for COF formation, as shown by the aminal COF, ${ }^{39,40}$ which is stable in both acid and base. The borazine linkage, isostructural to the boroxine and triazine linkages, was reported in $2012^{41}$ and the previously mentioned weak nitroso linkage in $2013 .{ }^{25}$ The following year, 2014, the first report of imide COFs appeared, ${ }^{42}$ for which the synthesis could be made reversible by carefully adjusting the basic catalyst and reaction conditions. ${ }^{29}$ The spiroborate linkage, ${ }^{43}$ reported in 2015 , provided a more stable B-O type linkage which is intrinsically anionic, with exchangeable counterions. However, while stable in aqueous and mild basic environments, the material was still susceptible to acidic conditions. In 2016, another major hurdle was overcome, by the synthesis of the fully conjugated acrylonitrile COF $2 \mathrm{DPPV},{ }^{44}$ based on $\mathrm{C}=\mathrm{C}$ linkages, which was reported to be stable in various solvents and concentrated acids and bases. ${ }^{45}$ Due to the strong electron withdrawing cyano groups, the formation of this strong bond could be made reversible. Two new linkages were reported in 2017 , the anionic silicate linkage, ${ }^{46}$ which showed the potential of the use of Si atoms in the synthesis of COFs but was unstable in aqueous environments and the highly stable amide linkage ${ }^{47}$ which could be made reversible at high temperatures $\left(>200{ }^{\circ} \mathrm{C}\right)$. While the amide linkage was already reported in 2016 via chemical conversion of imine linkages ${ }^{48}$ this was the first report of direct formation of amide bonds as COF linkage. It was observed that the materials were highly stable in both acid and base, outperforming even the most stable imine COFs and amide COFs formed via postmodification. The dioxin linked $\mathrm{COFs}^{49}$ COF-316 and COF-318 were, in 2018, the first COFs to be formed through an aromatic substitution reaction. Although the ring closing step could not be made reversible, crystalline COF formation was possible under highly specific conditions (by addition of nitrile side groups). The strong dioxin bonds formed resulted in high stability of the material in concentrated base or acid. Subsequently, COF-701, the first unsubstituted olefin linked COF was reported in 2019 and could be formed via an aldol condensation reaction. ${ }^{50,51}$ Stability tests showed the material withstanding both aqueous and organic solutions of strong base and acid. In the same year, an imidazole linked COF, chemically comparable to the previously mentioned benzoaxazole linkage, could be obtained by reaction of carboxylic acids and diamines. ${ }^{52}$ Finally, very recently the ester, ${ }^{53}$ pyrimidazole ${ }^{54}$ and polycubane ${ }^{55}$ linkages were added to the ever expanding library of COF linkages. Whereas the pyrimidazole linkage was shown to be stable in both $9 \mathrm{M} \mathrm{HCl}$ and $\mathrm{NaOH}$, the ester linkage was susceptible to both acids and bases but stable in aqueous environments. While more linkages have been claimed to be used in the synthesis of COFs, we choose to stick to the original definition of discoverer Omar M. Yaghi, saying that COFs should be permanently porous and crystalline, ${ }^{56}$ to create this list. Additionally, some linkages which only differ in functional group substitution like azine, ${ }^{57}$ indoimine $^{58}$ and 
urea $^{59}$ for imine, heptazine ${ }^{60}$ for triazine and Michael additionelemination ${ }^{61}$ for $\beta$-ketoenamine are not discussed.

1.1.2 Structural variety in COFs. These diverse linkages can be built into different framework structures, described by the underlying topologies. As topological representations only give information about connections, without being influenced by their chemical nature, it significantly reduces the complexity. ${ }^{62}$ Thus allowing both reverse engineering of materials ${ }^{63}$ and in depth structural study and comparison of materials. ${ }^{64}$ Different ways of COF representation are illustrated in Fig. 3 for COF-300. A only gives chemical information (chemical representation) and is frequently used in polymer chemistry. B, the framework representation, contains both chemical and structural information, therefore this representation will typically be used for the remainder of this review. Finally, C, shows the topological representation or net. The same geometrical information can be distinguished as in $\mathbf{B}$, but due to the removal of chemical information, analysis is more straightforward.

These nets can be described as a collection of nodes and edges, with the difference between edge and node determined by the number of connections made. Edges can make exactly two connections, as e.g. the terephtaldehyde (BDA) linker in COF-300 (Fig. 3A and B). COF nodes can be defined as linkers making three or more connections, in our COF-300 example, tetrakis(4-aminophenyl)methane (TAM) is the node. COF-300 only has one kind of node (uninodal) and one kind of edge (edge-transitive), ${ }^{65}$ later on examples with multiple edges or nodes will be shown. Additionally to the number of connections, the local symmetry is important too, for example a tetratopic node (four connections) can be described as a tetrahedron (as in COF300 ), square plane or reduced symmetry square plane (all three possibilities are drawn in Fig. 4). Finally, the connections between edges and nodes and the respective spatial arrangement co-define the formed net. ${ }^{66}$ This indicates that multiple nets can be formed by one edge/node combination, resulting in a vast amount of possible nets (2927 3D nets and $2002 \mathrm{D}$ nets could be found in the Reticular Chemistry Structure Resource, RCSR). ${ }^{64}$ However, only very few make up for the vast majority of reported structures. ${ }^{67}$

Until now, 23 nets have been reported for COFs divided in nine $2 \mathrm{D}$ layered structures and 14 3D nets. The formed nets as well as some edge/node combinations used to obtain these are presented in Fig. 4. As most linkers are largely constructed from aromatic rings and $\mathrm{sp}^{2}$-hybridized atoms for reasons of rigidity, strong $\pi-\pi$ interactions make the crystallization of $2 D$ layered structures favorable. ${ }^{65}$ Among the 2D layered structures, the lion's share of COFs crystallize in one of the five edge-transitive nets. Of those, four nets are uninodal and only the kgd net is binodal, as it combines a hexatopic and a tritopic node. ${ }^{68}$ The uninodal 2D nets are the hexagonal hcb net, ${ }^{1}$ the sql net constructed from square tetratopic linking units, ${ }^{69}$ the hxl net with triangular pores ${ }^{70}$ and the kgm net, ${ }^{71}$ which is obtained by the linking of tetratopic building units with reduced symmetry (respectively $120^{\circ}$ and $60^{\circ}$ between the points of extension instead of $90^{\circ}$ ) like $4,4^{\prime}, 4^{\prime \prime}, 4^{\prime \prime \prime}$-(ethene-1,1,2,2-tetrayl)-tetraaniline (ETTA). Lately, efforts have been made to increase the structural diversity of 2D COFs, resulting in the reports of COFs based on the $\mathbf{m t f},{ }^{72} \mathbf{f x t},{ }^{73} \mathbf{b e x}^{74-76}$ and $\mathbf{t t h}^{77}$ non-edge-transitive $2 \mathrm{D}$ nets. At this point it is important to note that the relationship between node geometry and topology of the resulting COF is not as straightforward as it seems. Not only can the same net be formed by different node combinations (for example a sql net can be formed by the condensation of a tetratopic node with an edge, or two tetratopic nodes), also some combinations can in theory lead to different nets (the combination of a desymmetrized tetratopic linker with a tritopic linker has for example already been reported to crystallize in $\mathbf{m t f}$ and bex topologies). It is not always possible to predict what topology will be formed, although a general rule of thumb exists: "the materials have a tendency to crystallize in the symmetrical topology possible and will form $2 D$ layered structures whenever possible.",78

Theoretically, structural diversity is much larger for 3D than 2D structures. However, until now, only 14 nets have been reported in the synthesis of 3D COFs, which can be explained by the increased difficulty in crystallizing these compounds in combination with the reduced stability and need for polyhedral linkers. ${ }^{79}$ Almost half of these nets are constructed using a tetrahedral node, with the dia topology being by far the most common. ${ }^{32}$ These networks are obtained by reticulation of a tetrahedral node with a linear linker or another tetrahedral node.
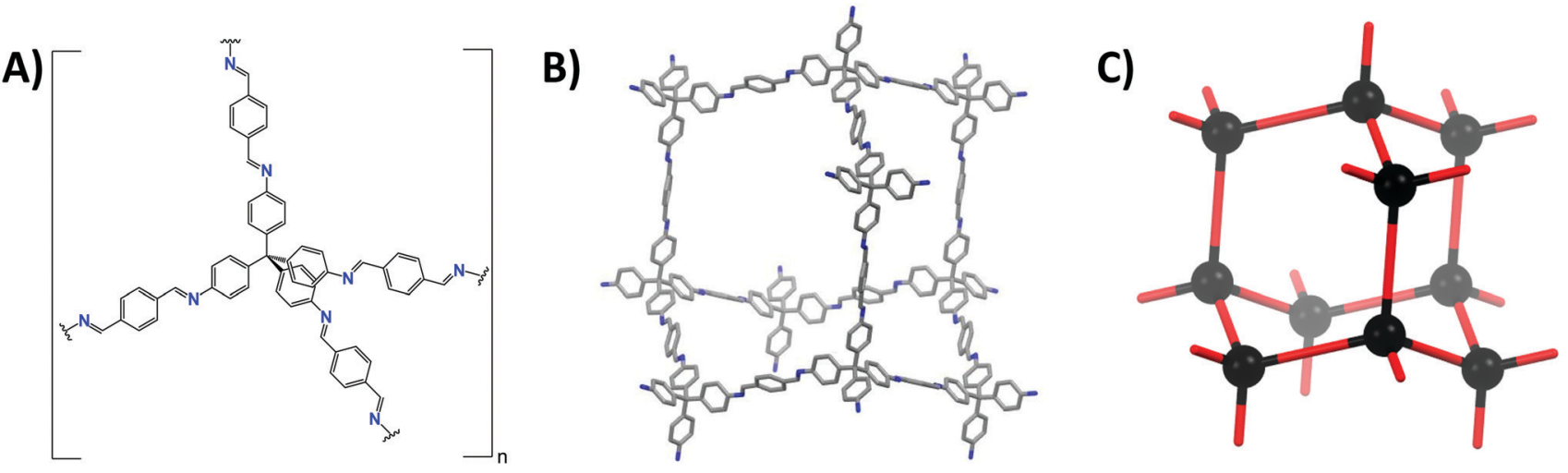

Fig. 3 Different ways of COF presentation, illustrated for COF-300. (A) Chemical representation. (B) Framework representation showing exactly one pore. (C) Topological representation, nodes are shown as black dots, edges as red lines. 


\section{D nets}
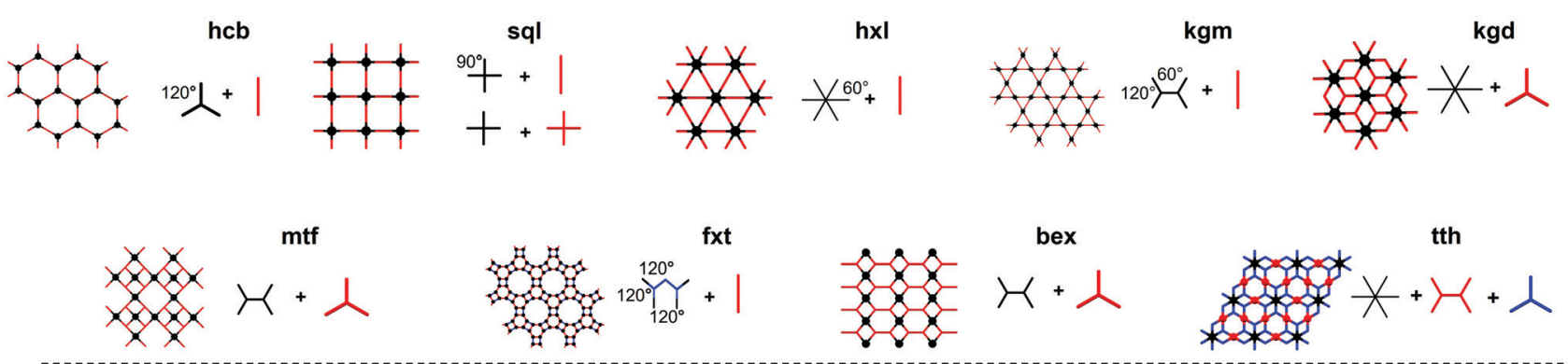

\section{D nets}
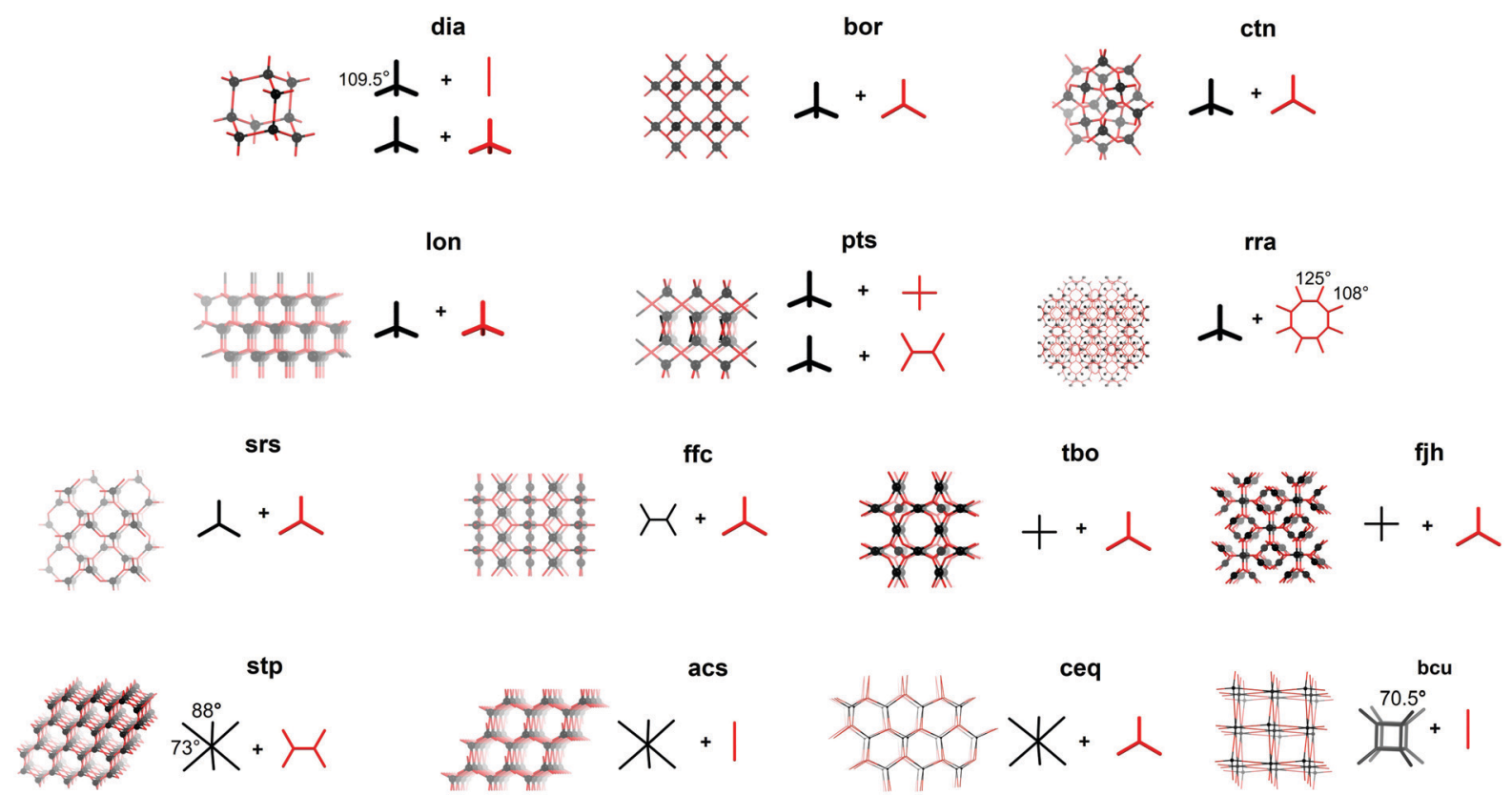

Fig. 4 Different topologies reported in COF chemistry, building units are shown in different colors (black, red and blue). Some examples of possible geometric combinations (+ required angles) leading to the desired net are included.

However the latter combination can also result in a material with lon topology. ${ }^{3}$ Combination of these tetrahedral nodes with a tritopic linker can give both ctn or bor networks, where the main difference is the topological density (number of vertices per unit volume) of the networks. ${ }^{23}$ The pts net could be obtained by the reaction of $\mathrm{C} 4$ linkers with tetrahedral nodes, ${ }^{80}$ while the rra topology was accessed by combining a tetrahedral node with $\gamma$-cyclodextrin. ${ }^{81}$ It is important to note that these networks can also be formed through molecular weaving, as the structuredefining metal complex in a woven framework can essentially be seen as a tetrahedral node. This way, woven networks of dia and pts topology have already been prepared. ${ }^{26,82,83}$

From 2018, COFs crystallizing in 3D nets without the use of tetrahedral nodes where found, like the srs net built of two C3 linkers. However, the $\left[\mathrm{SiO}_{6}\right]^{2-}$ linker also coordinates two $\mathrm{Na}^{+}$cations, making the linker chiral and non-coplanar. ${ }^{84}$ Combination of planar C4 and C3 nodes can lead to materials in different topologies, of which a rich library exists in the metal organic framework (MOF) world. However, only three 3D
COF topologies (ffc, ${ }^{85}$ tbo $^{86}$ and $\mathbf{f j h}^{87}$ ) have been reported based on this combination of linkers. Recently, trigonal prismatic nodes have been used in 3D COF synthesis, resulting in nets with the stp, ${ }^{88}$ acs $^{89,90}$ and ceq $^{90,91}$ topology. Finally, the bcu net could be obtained by using the aforementioned polycubane linkage as a octahedral node. ${ }^{55}$

The structural diversity of COFs goes beyond the underlying topologies, for example isoreticular expansion and contraction is an often studied concept which can have large implications on the structure. Some 3D structures (like dia and pts topologies) are prone to interpenetration, which can be significantly altered by expanding or contracting the used linkers. ${ }^{78,79}$ In $2 \mathrm{D}$ structures, stacking modes can have a huge influence on properties, varying from $\mathrm{AA}$ over $\mathrm{AB}$ to even $\mathrm{ABC}^{\prime}$ stacking modes. ${ }^{92,93}$ Moreover, layer stacking can be influenced by solvent adsorption ${ }^{94}$ or linker substituents. ${ }^{95}$ Additionally, linker desymmetrization can significantly alter the framework, without changing the underlying topology. Using this principle, COFs crystallizing in a hcb net with hierarchical $^{96}$ or brick-wall ${ }^{97}$ pores could be obtained. 


\section{The crystallization problem}

As already shortly discussed above, one of the main issues in the world of COFs is the crystallization problem, which essentially means that the more stable a solid compound is, the harder it is to crystallize (Fig. 5A). For example, compounds held together by weak interactions as van der Waals forces and hydrogen bonds, will crystallize relatively easily, while coordination networks are already more complicated to crystallize. However, it really gets problematic once you get into the domain of reticular chemistry. ${ }^{99}$ To obtain such extended crystalline solids, like MOFs and COFs, it is essential to have reversible linkage formation, on a time scale that allows for error-correction, whereas the formation of covalent bonds is generally already irreversible under very mild conditions. ${ }^{100}$ As a consequence, while MOF single crystals are still regularly reported, COF crystallization is still highly problematic. So, the trick is to make the formation of those highly stable linkages more reversible, as this will give more room for error-correction and thus make the crystalline product more accessible. ${ }^{78}$ Practically, this is done using the principles of DCC, as shown in Fig. 5B. In contrast to most synthetically used reactions, DCC allows a covalent bond to be broken and reformed, making error-correction possible. During reaction, products will gradually equilibrate from the kinetic (amorphous) product, rapidly formed due to the low activation barrier $\left(\Delta G^{i}\right)$, to the thermodynamically stable (crystalline) product with the largest reduction in standard Gibbs free energy $\left(\Delta G^{0}\right){ }^{98}$ This also makes it possible to control the reaction by introducing stabilizing features for the desired product and driving the reaction equilibrium by using excess starting materials, modulators or removing condensation products. ${ }^{18}$ As a consequence, a detailed understanding of the different reaction states in the formation of COFs will yield higher control over the crystallinity of the product. Still, it has to be noted that the polymerization and resulting precipitation of COFs is a complicating factor, as precipitated amorphous networks might get kinetically trapped. Especially 3D COF formation, fully constructed from covalent bonds, is hindered by this problem, whereas the $\pi-\pi$ stacking interactions in 2D COFs help driving the reaction to a layered structure. ${ }^{101}$

\subsection{Mechanism of boroxine COF formation}

As the first COFs were based on boroxine and boronate ester chemistry, much research has been done on elucidating the formation mechanism of these materials. The underlying chemistry is quite straightforward, driven by Lewis acidic dimer formation between oxygen and boron atoms of two $\mathrm{B}(\mathrm{OH})_{2}$ moeities (with expulsion of a water molecule). This process repeats three times, until a $\mathrm{B}_{3} \mathrm{O}_{3}$ ring and three $\mathrm{H}_{2} \mathrm{O}$ molecules are formed. ${ }^{105}$ As the boron atoms retain their Lewis acidic character and can easily be attacked by oxygen in e.g. $\mathrm{H}_{2} \mathrm{O}$, the reaction is completely reversible However, precipitation of oligomers and crystallites severely complicate the mechanism and analysis of boroxine COF formation, therefore requiring more in-depth research. A first mechanistic study bypassed this issue by adjusting the solvent combinations until fully homogeneous growth conditions could be obtained for COF-5 (by using 4/1 dioxane/mesitylene as solvent with 15 eq. methanol to the catechol as solvent), making it possible to track the COF formation via turbidity measurements. ${ }^{102}$ A reproducible induction period was observed, which is followed by gradual precipitation of the $\mathrm{COF}$ as evidenced by an initial linear increase of turbidity in the COF mixture. Moreover, as shown in Fig. 6A, the induction period and formation rate are readily influenced by
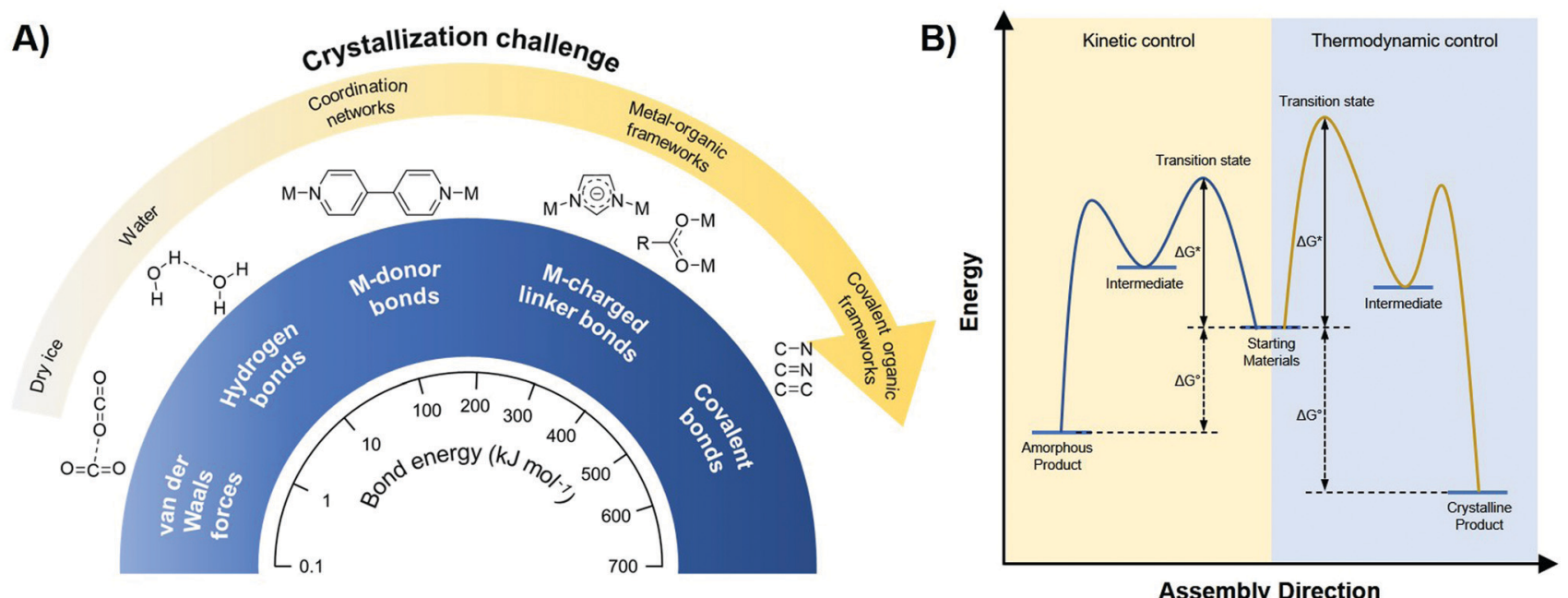

Fig. 5 (A) Relationship between bond strength and crystallization challenge for different interactions. When going from weak (van der Waals forces, hydrogen bonds) over intermediate (metal-donor bonds) to strong (metal-charged linker bonds, covalent bonds) interactions, the crystallization challenge increases dramatically. (B) Free energy profile illustrating the difference between kinetic (green) and thermodynamic (red) control. When a reaction is kinetically controlled (like most covalent reactions), it is irreversible and amorphous products will be formed. Thermodynamically controlled reactions (like supramolecular chemistry and dynamic covalent chemistry) are reversible and can yield crystalline materials. Reproduced and adapted with permission from ref. 98. Copyright MDPI (2017). 

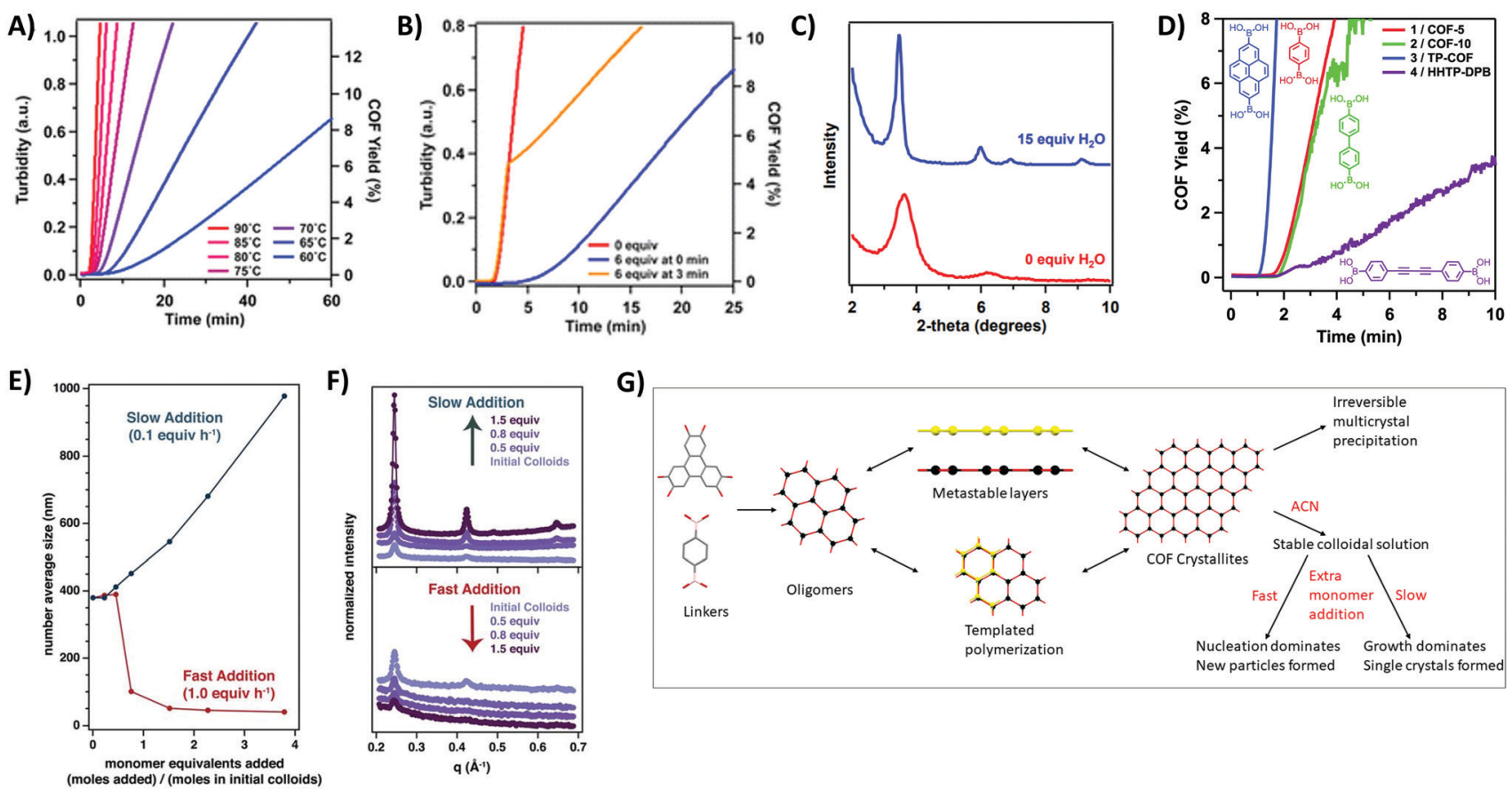

Fig. 6 Mechanistic studies on COF-5. (A) Turbidity increase over time tracked at various temperatures. Highest temperature $\left(90{ }^{\circ} \mathrm{C}\right)$ is indicated in red, lowest $\left(60^{\circ} \mathrm{C}\right.$ ) in blue. Turbidity is directly proportional to the rate of COF precipitation. (B) Influence of modulator (4-tert-butylcatechol) addition on COF synthesis, equivalents are taken relative to the amount of 2,3,6,7,10,11-hexahydroxytriphenylene (HTTP). Curve without modulator is shown in red, addition of 6 eq. of modulator at zero minutes is presented in blue and the orange curve shows addition of 6 eq. of modulator at three minutes. (C) Partial PXRD pattern for COF-5 synthesized with 0 (red) or 15 (blue) eq. of additionally added $\mathrm{H}_{2} \mathrm{O}$. (D) COF Formation over time for four different boronate COFs (COF-5 in red, COF-10 in green, TP-COF in blue and HHTP-DPB in purple). All four COFs are built from the condensation of HTTP with the linker drawn next to the curve. (E) DLS average size of COF- 5 particles as a function of the added monomer concentration. Slow addition ( 0.1 eq. per $h$ ) is shown in blue, fast addition (1.0 eq. per h) in red (F) WAXS patterns of COF-5 particles. The upper graph shows the influence of slow monomer addition, the lower graph the influence of fast monomer addition. (G) Proposed mechanism of formation for COF-5 using combined information from available mechanistic studies. Linkers react readily to oligomers, which can stack to metastable layers or go through templated polymerization processes. Eventually COF crystallites form, which precipitate immediately to chemically unavailable multicrystals if not stabilized by nitrile solvents as ACN. Stable colloidal COF solutions can be further reacted by the addition of new monomers. If these monomers are added in a rapid way, nucleation dominates and new (small) particles are formed, but if these additional monomers are added slowly, growth dominates and single crystals are formed. Reproduced with permission from: (A-C) ref. 102, Copyright American Chemical Society (2014); (D) ref. 103, Copyright Royal Society of Chemistry (2015); (E and F) ref. 104, Copyright American Association for the Advancement of Science (2018).

temperature, with lower temperatures leading to longer induction periods and slower COF formation. The influence of modulation on the formation of COF-5 was also studied (Fig. 6B), it can be observed that as soon as a modulator is added, the COF formation slows down, indicating that competing reactions are indeed happening. However, as no modulator was incorporated in the final COF structure and the isolated yields with and without modulator were very comparable, it could be concluded that an irreversible step is present in the reaction mechanism. Afterwards, even addition of large amounts of modulator was not sufficient to decrease turbidity, indicating chemical inaccessibility of these crystallites. Finally, the effect of $\mathrm{H}_{2} \mathrm{O}$ addition was studied, which could theoretically inhibit $\mathrm{COF}$ formation via Le Châtelier's principle. Indeed, addition of 15 eq. $\mathrm{H}_{2} \mathrm{O}$ afforded larger crystallites as evidenced by the sharper (100) Powder X-Ray Diffraction (PXRD) peak at a $2 \theta$ value of $3.5^{\circ}$ ( $\mathrm{Cu} \mathrm{K} \alpha$ radiation) shown in Fig. 6C. Nonetheless, a practical limit for this method of crystal growing exists, as adding 20 eq. of $\mathrm{H}_{2} \mathrm{O}$ resulted in little to no COF formation after several days. These results indicate that efficient control of temperature and $\mathrm{H}_{2} \mathrm{O}$ in the reaction, as well as modulator addition can vastly improve the synthesis of boroxine and boronate COFs. Later on, the same procedure was used to study the difference in the kinetics of COFs made by different linkers. ${ }^{103}$ Two different effects can be observed from Fig. 6D, namely an increase in formation rate when the aromatic domains of the linker increases and a decrease in formation rate with increasing pore size. The first, and most important effect was attributed to stabilization of the intermediate by layer stacking, while the second could be explained by the decrease in interlayer attraction. Thus, it could be concluded that the rate determining step in the formation of 2D COFs is the interlayer stacking, yielding strong interactions and faster precipitation. Theoretical studies on the formation of boronate ester COFs have also been done, tracking the crystallization of COF-5 via kinetic Monte Carlo simulations, ${ }^{106}$ free energy techniques ${ }^{107}$ and molecular dynamics simulations. ${ }^{108}$ Firstly, linkers stack and form oligomers, a process that needs heating. Subsequently, these oligomers can nucleate via two different mechanism, i.e. stacking of metastable layers and templated polymerization. It should be noted that all these steps are fully reversible and 
exchangeable (as shown in Fig. 6G). However, due to the strong stacking interactions between the linkers, these processes cause a tremendous amount of hard to anneal defects, which limit COF crystallinity. It has been shown that appropriate solvents can greatly reduce the amount of effective stacking interactions and therefore increase crystallinity. ${ }^{107}$ Moreover, addition of $\mathrm{H}_{2} \mathrm{O}$ and methanol can vastly increase reversibility of the boronate ester formation as these molecules stabilize the transition state from a highly strained 4-ring to a more stable 6-ring, therefore lowering the activation energy from $25 \mathrm{kcal} \mathrm{mol}^{-1}$ to 13 or $14 \mathrm{kcal} \mathrm{mol}^{-1}$, respectively. Hence for the growth of large COF crystals the irreversible agglomeration and subsequent precipitation of small crystallites are problematic (as can be noticed in the proposed model in Fig. 6G).

A solution for this was reported in 2017 , when the Dichtel group succeeded in stabilizing COF- 5 colloids by the addition of small volume fractions of nitrile solvents (like acetonitrile, ACN). ${ }^{109}$ The obtained colloids were stable for over a month and were shown to be crystalline by Wide/Small-Angle X-ray Scattering (WAXS/SAXS) measurements. While the particle size was shown to be dependent on the amount of added nitrile solvent (with larger colloids forming at lower vol\% due to aggregation of small crystallites), no change in particle size was observed when the solvent composition was altered after colloid formation. This indicated kinetic trapping of the COF in the formed colloids. Moreover, the reported procedure could be extended to a range of different linkers (DBD-COF, BPh-COF, Py-COF), 3D materials (COF-105, COF-108) and boroxine linked COFs (COF-1, COF-102, COF-103). ${ }^{110}$ In 2018, these kinetically trapped colloids were used for the synthesis of large single crystals of COF-5, COF-10 and TP-COF, by the slow addition of monomers. ${ }^{104}$ From Fig. $6 \mathrm{E}$ and F, it can be observed that when monomers were added to these stabilized colloids at a rate of 0.1 eq. per hour both crystallinity and particle size improved, as could be measured by Dynamic Light Scattering (DLS) and WAXS. However, when fast monomer addition was tested, particle size and crystallinity diminished, most likely because the critical nucleation concentration was reached. This indicates the importance of effective nucleation and growth separation for the synthesis of large COF crystals. Recent kinetic Monte Carlo simulations supported these results, as second order kinetics were obtained for nucleation while growth processes were calculated to be of a pseudo-first order in monomer concentration. ${ }^{111}$ However, crystal growth by keeping the monomer concentration below the critical value for crystal expansion is impractical, as a quadratic dependence on time was observed which corresponds to time ranges for the growth of millimeter sized crystals in the order of millions of years.

\subsection{Mechanism of imine COF formation}

Imine chemistry was, in contrast to boroxine chemistry, already much more studied before the first reports of COFs based on this type of linkages were reported. For example, these reactions were already widely used in $\mathrm{DCC}^{114}$ and Schiff base synthesis. ${ }^{115}$ Therefore, three reversible reactions happening simultaneously were already identified, namely imine condensation, exchange and metathesis (Fig. 7E). Interestingly, imine exchange was found to happen more rapidly than imine condensation, ${ }^{116}$ making amine modulators very interesting
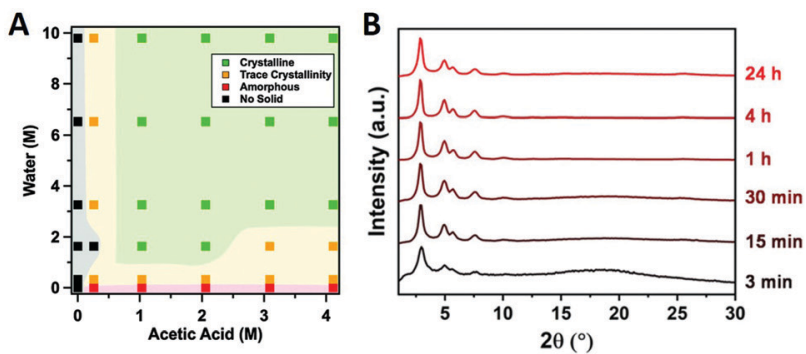

E

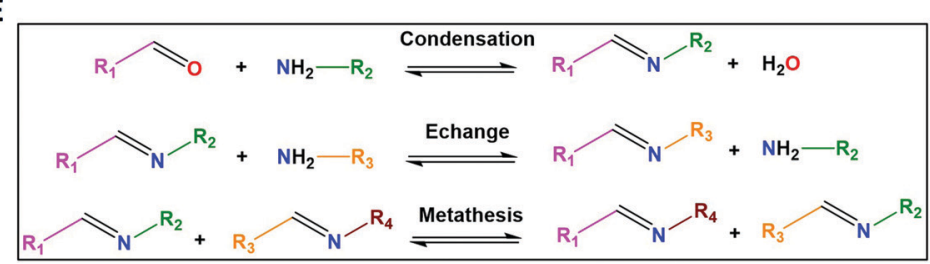

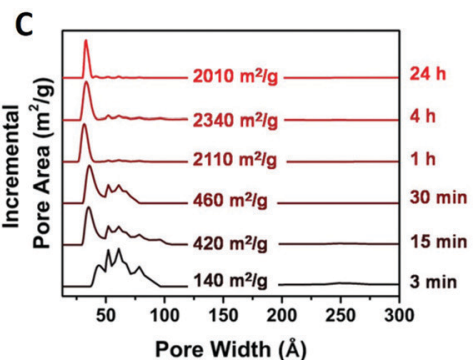

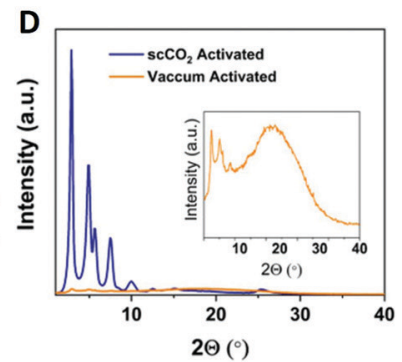

$\mathbf{F}$

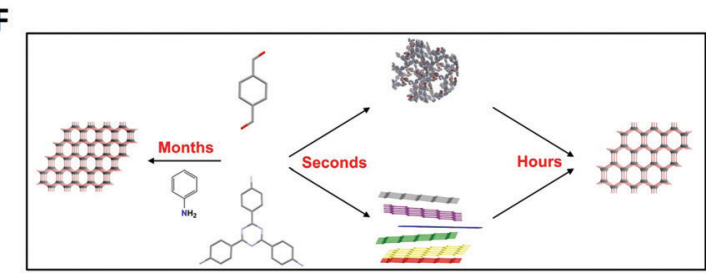

Fig. 7 Mechanistic studies on imine COFs. (A) TAPB-BDA COF formation (4/1 dioxane/mesitylene, $70{ }^{\circ} \mathrm{C}, 3 \mathrm{D}$, vacuum activation) as a function of $\mathrm{H}_{2} \mathrm{O}$ and acetic acid concentration. Shaded areas are added as a guide for the eye. (B) Normalized PXRD patterns of TAPB-BDA COF (4/1 dioxane/mesitylene, $70{ }^{\circ} \mathrm{C}, 33$ vol\% $10.5 \mathrm{M}$ acetic acid, $\mathrm{scCO}_{2}$ activation) in function of time. (C) Normalized Pore Size Distributions and $\mathrm{S}_{\mathrm{BET}}$ values of TAPB-BDA COF (4/1 dioxane/mesitylene, $70{ }^{\circ} \mathrm{C}, 33$ vol\% $10.5 \mathrm{M}$ acetic acid, $\mathrm{scCO}_{2}$ activation) in function of time. (D) PXRD pattern of TAPB-BDA COF (4/1 dioxane/ mesitylene, $70{ }^{\circ} \mathrm{C}, 33 \mathrm{vol} \% 10.5 \mathrm{M}$ acetic acid, $4 \mathrm{~h}$ ) using vacuum (yellow) and $\mathrm{scCO}_{2}$ (blue) activation. (E) Overview of the chemical mechanism of imine COF formation, the three types of imine reactions: imine condensation, imine exchange and imine metathesis. (F) Schematic drawing of the physical mechanism of imine COF formation. When linkers are combined in presence of large excess of aniline single crystal COFs can form over months. Alternatively, amorphous polymers or disordered sheets are rapidly formed (within seconds) and subsequently slowly rearranged to COF multicrystals (hours). Reproduced with permission from: (A) ref. 112, Copyright Royal Society of Chemistry (2016); (B-D) from ref. 113, Copyright John Wiley and Sons (2019). 
candidates to improve error-correction, as they provide extra functionalities capable of imine exchange. In 2015, a first mechanistic study of imine COFs was published by the Dichtel group. ${ }^{112}$ They concluded that both $\mathrm{H}_{2} \mathrm{O}$ and acetic acid are indispensable in the synthesis of the studied TAPB-BDA COF (TAPB: 1,3,5-tris(4-aminophenyl)benzene). From Fig. 7A, it is clear that no solids are obtained when COF synthesis is attempted without added acetic acid, while absence of $\mathrm{H}_{2} \mathrm{O}$ leads to amorphous powders. High yields and crystallinity could be obtained only when $\geq 3.3 \mathrm{M} \mathrm{H}_{2} \mathrm{O}$ and $\geq 1 \mathrm{M}$ acetic acid were added, but increasing the concentrations above this threshold did not improve the quality of the obtained COF. Moreover, a two-step mechanism was observed, with rapid precipitation of an amorphous solid followed by dynamic imine exchange to finally obtain a crystalline material after a few days. As Fourier-transform infrared spectroscopy (FTIR) analysis of the formed amorphous solid did not show any amine or aldehyde peaks and the yield was already 95\% after 15 minutes, it was concluded that no free monomers or oligomers were needed to achieve this amorphous to crystalline transformation. This theory was further supported by the possibility to grow crystalline COFs from isolated and cleaned amorphous solids. ${ }^{112}$ Additionally, it was proven possible to recrystallize an amorphous polyimine (coated around $\mathrm{Fe}_{3} \mathrm{O}_{4}$ nanoparticles) to a crystalline COF without changing the morphology or particle size. ${ }^{117}$ However, quite recently, an alternative pathway was proposed based on the observation of crystalline lattice fringes in Transmission Electron Microscopy (TEM) samples of COFs prepared in reaction times as short as 60 seconds. As the observed sheets were very thin, it was suggested that COFs might initially crystallize as disordered sheets, followed by a recrystallization to ordered and porous powder. ${ }^{118}$ Moreover, equilibration of these 60 seconds samples in supercritical $\mathrm{CO}_{2}$ $\left(\mathrm{scCO}_{2}\right)$ for 72 hours led to crystalline peaks as observed by PXRD. The difference between both proposed mechanism is illustrated in Fig. 7F.

Additionally, a significant influence of activation methods was observed, with crystalline COFs obtained (via in situ X-ray diffraction) at reaction times as short as 90 seconds while common synthetic conditions required three days of reaction time. This could be explained by pore collapse during vacuum drying of COFs. However, when activation was performed using $\mathrm{ScCO}_{2}$, highly crystalline and porous imine COFs could be readily synthesized at short reaction times (four hours), as shown in Fig. 7B and C. ${ }^{113}$ The difference between $\mathrm{scCO}_{2}$ and vacuum activation for TAPB-BDA COF is illustrated in Fig. 7D. Still, not all imine COFs suffer from these pore collapse processes, with more robust materials taking advantage of alkoxy side-groups or enlarged $\pi-\pi$ systems in the linkers. ${ }^{119}$ Furthermore, it was shown that framework damage for fragile materials was (partially) reversible by treatment with $\mathrm{scCO}_{2}$, while FTIR spectra were unaffected by the activation method, indicating that these effects could be attributed to physical processes. ${ }^{113,119}$ Recent Density-Functional Theory (DFT) calculations seemed to partially explain these observations, as it was observed that $\pi-\pi$ layer interactions are not strong enough in solution leading to the coexistence of different stacking modes and thus disorder. Materials with large pores and small p-conjugated structures (fragile) were shown to be prone to random rearrangement. Finally, as quite expensive equipment is needed for $\mathrm{scCO}_{2}$, a cheaper but reliable alternative was developed in a sequential solvent exchange protocol, going from high to low surface tension, followed by drying under $\mathrm{N}_{2}$-flow with a gentle temperature ramp. Recently, it has been shown that immersion in an ultra low surface tension solvent (like perfluorohexane) followed by vacuum drying at $80{ }^{\circ} \mathrm{C}$ is sufficient to retain crystallinity and porosity. ${ }^{120}$

At this point, it is important to note that all the previously described mechanistic studies have been performed only on 2D imine COFs. Very little is known about the mechanism of 3D imine COF formation, but it has been proven that the formed amorphous intermediate can be recrystallized to crystalline COF-300. ${ }^{121}$ As no layers exist for these 3D structures, the most plausible mechanism is via the amorphous polymer. Moreover, the more open pores, lacking $\pi-\pi$ layer interactions are highly susceptible to pore collapse when common activation methods are used. Finally, additional complicating factors exist for 3D COFs, like interpenetration of networks and hydration causing pore collapse. $^{3}$

Still, up to date, the only COFs of which single crystals suitable for SXRD were obtained are 3D imine COFs. ${ }^{3}$ This was achieved by the addition of large amounts of aniline as modulator, slowing down the synthetic process to 30-80 days (this process is shown schematically in Fig. 7F). As aniline has comparable reactivity to the building blocks, it is an ideal modulator, shifting the COF formation from rapid nucleation with limited growth (resulting in amorphous or polycrystalline compounds) to an equilibrium by addition of sufficient amounts of the modulator. The increased amount of amine in the solution leads to increased error-correction (by fast imine exchange) and slow formation of COFs as single crystals, a process schematically explained in Fig. 11A. For example, the addition of 15 eq. of aniline led to COF-300 crystals of $60 \mu \mathrm{m}$ in $30-40$ days, allowing structure solution by synchrotron SXRD analysis. The difference in morphology for polycrystalline and single-crystal COF-300 is illustrated by SEM images in Fig. 11B. Four different 3D imine COFs, forming two nets (dia and lon) could be crystallized and analyzed via this method. A recent follow-up paper also reported the synthesis of $50 \mu \mathrm{m}$ crystals of a COF crystallizing in the pts topology using 50 eq. aniline although the quality of the obtained crystals was not sufficient to allow SXRD structure solutions. ${ }^{122}$

\subsection{Practical significance of crystallinity}

Given the substantial challenges associated with getting highly crystalline COFs, it might be attractive not to spend the effort and settle for moderately crystalline or even amorphous products. However, in some cases like optoelectronics and photocatalysis, high crystallinity is of essential importance in order to get desired applications to work effectively.

For example, several years of research in conjugated polymers have shown that parameters such as lattice constant and 
crystallinity are critical for determining the resulting charge transport properties of the semiconducting polymers. ${ }^{123}$ The mobile charge carrier travels at certain velocities in the backbone of an ordered crystalline polymer and hops into the disordered regions causing them to change the velocities. Microstructure tuning focuses on reducing the probability of charge transport under unfavorable conditions and improve the mobility in certain parts of the polymer. It has been observed that mobilities are enhanced in crystalline phases with sufficient intercrystallite connectivity. ${ }^{124}$ Synthetic advancements in conjugated polymers to obtain high crystallinity and long-range order have made poly(3-hexylthiophene) (P3HT) the most widely studied conjugated polymer due to high mobilities above $0.01 \mathrm{~cm}^{2} \mathrm{~V}^{-1} \mathrm{~s}^{-1} \cdot{ }^{125}$ Compared to previous transistors using amorphous polythiophenes the mobilities in the crystalline P3HT based transistors increased by a factor of 100. Further improvement in the crystallinity was achieved using a polythiophene derivative, poly(bithiophene-alt-thienothiophene) (PBTTT) ${ }^{126}$ Transistors based on PBTTT displayed even further enhanced mobilities above $0.1 \mathrm{~cm}^{2} \mathrm{~V}^{-1} \mathrm{~s}^{-1}$. Naturally, the charge mobilities also depend on other factors such as the chemical functionalities in the polymer, but crystallinity is certainly considered one of the most crucial components.

In the field of nanoelectronics, 2D materials have played a major role due to high charge carrier mobility and tunable band gaps. ${ }^{127,128}$ These properties can be tuned based on the choice of the molecular building blocks of the 2D materials. ${ }^{129}$ Computational studies have predicted the electronic structures of several 2D materials to exhibit Dirac cones which yield massless charge carriers and flat bands which can quench the kinetic energy of charge carriers. ${ }^{130}$ These have important consequences in several interesting phenomena such as anomalous Hall effect and surface superconductivity. ${ }^{131,132}$ However, the experimental observation of these electronic structures have been limited due to constraints in domain size and defect density of the 2D materials. Recently, mesoscale ordered 2D conjugated polymer have been synthesized which have experimentally confirmed the predicted electronic structures. ${ }^{133}$ However, the advancements in computational analysis of such materials have been a step ahead and need to be matched with experimental outputs for advanced applications. In essence, materials with higher crystallinity can reciprocate the predicted electronic properties.

Charge carrier transfer processes play an essential role in photocatalytic reactions. ${ }^{134}$ Upon light irradiation with sufficient energy on a semiconductor surface, electron-hole pairs are generated. Electrons are excited into the conduction band from the valence band by absorption of the energy from light irradiation. Photocatalysis is then carried out through the participation of the generated photoelectrons or holes, for reduction or oxidation, respectively. However, a major challenge hindering the efficiency of the overall photocatalytic process is recombination of the generated electrons and holes. Every charge carrier generated through light irradiation is associated with a carrier lifetime. To proceed with the desired catalytic reaction, the electrons and holes must be kept apart and be utilized immediately. Empirical evidences suggest that amorphous (defective) regions in semiconductors often act as recombination centers and are detrimental to charge transport. ${ }^{135,136}$ Hence, in general, crystalline (defect-free) materials are engineered in order to decrease the overall density of recombination centers thus enhancing the carrier lifetimes of photogenerated carriers. Lin et al. ${ }^{137}$ compared the effect of crystallinity of graphitic carbon nitride ( $\mathrm{g}-\mathrm{CN})$ polymers on photocatalytic hydrogen evolution reaction and oxygen evolution reaction. In both reactions, g-CN-1 (enhanced crystallinity) showed better performance than bulk g-CN (moderate crystallinity). Photoluminescence spectroscopy confirmed an increase in the charge carrier separation and decrease in the recombination rates of the photoexcited carriers in g-CN-1 compared to the bulk g-CN. Recently, COFs have emerged as potential candidates for several photocatalytic transformations. ${ }^{138-141}$ Pachfule et al. ${ }^{138}$ showed that a crystalline diacetylene functionalized COF performs better than its amorphous counterpart in photocatalytic hydrogen evolution reaction. Hence, it is clear that crystallinity is a crucial component in improving the charge carrier separation and transfer kinetics.

\subsection{Analyzing crystalline compounds}

Apart from having an influence in the material properties, crystallinity of the material is also related to the ease of characterization. In general, crystalline materials are easier to decipher and correspondingly provide more insight into their structural properties. An important feature that makes COFs unique compared to other porous organic polymers is their crystallinity which allows easier characterization. Currently, the go-to method for analysis of the COF structure is PXRD. In theory, it is possible to directly obtain a structure solution from PXRD, using a process involving peak indexing, space group determination, structure solution and final Rietveld refinement. ${ }^{142-144}$ However, due to the 1D projection of PXRD, a significant amount of data is lost, especially for high symmetry structures as COFs. ${ }^{142,144}$ Therefore, direct structure solution of PXRD data has not been reported yet for COFs. Still, the rigidity of COF linkers allows us to accurately predict the topology of the resulting material based solely on the used linkers (as explained in Section 1.1.2). This makes it possible to solve the structure of COFs by building models based on the most feasible topologies and refining those against the experimental powder pattern. Even when multiple topologies ${ }^{23,90,91}$ or interpenetration modes ${ }^{32,80}$ are possible, this should result in one single structure solution (when the quality of the experimental data is sufficient) because of the basic postulate of structural crystallography: "only one chemically sound crystal structure exists that is compatible with the experimental diffraction data". ${ }^{143}$ Additionally, PXRD patterns can be used to estimate the quality of the COF via crystallite size and degree of crystallinity. For example, the FWHM (Full Width at Half Maximum) of the PXRD reflections can be used to estimate the crystallite size via the Scherrer equation, ${ }^{145}$ with sharper reflections indicating larger crystallites and thus increased quality. In turn, the ratio of crystalline (sharp) peak area versus 
total peak area gives the degree of crystallinity. ${ }^{146}$ However, a thorough and true knowledge of the chemical nature of the synthesized COF is not assured using PXRD structure solution. For example, the structure of COF-300 in the powder form $(\text { dia-c5 })^{32}$ was slightly different than COF-300 in single crystal form (dia-c7). Even though the dia-c5 structure of the COF-300 was verified through PXRD characterization, there was some ambiguity in the degree of interpenetration. This ambiguity was eventually cleared through the single crystal XRD characterization of the COF-300 crystal. $^{3}$ Controlled polymerization leading to highly crystalline COFs has also enabled detailed structural analysis through synchrotron XRD and high-resolution transmission electron microscopy (HR-TEM). ${ }^{110}$ Recently, electron diffraction methods have also been utilized to solve the structure of crystalline COFs. ${ }^{147,148}$ To maximize comparison and reproducibility of COF analysis, we kindly refer to the guidelines on sample preparation and data treatment as given in "Standard Practices of Reticular Chemistry". ${ }^{56}$ Some useful complementary techniques, like solid-state nuclear magnetic resonance spectroscopy (ss-NMR) and FTIR are also listed in this work.

\section{Approaches towards high-quality COFs}

Several tactics have already been used to improve both crystallinity and porosity of COFs, resulting in higher quality materials. In the following we divide the used methods in seven different categories, of which the five most important ones are schematically illustrated in Fig. 8. It has to be noted that some papers reported combinations of different methods, if so we have put the discussion where most appropriate.

First of all, and already applied successfully in the synthesis of 3D COF single crystals, ${ }^{3}$ is the insertion of monofunctional molecules. This process is called modulation and is expected to decrease nucleation as well as increasing error-correction.

Next is the decrease of monomer availability, which leads to slower reactions with less defects. This method can be performed using physical slow monomer addition (as shown

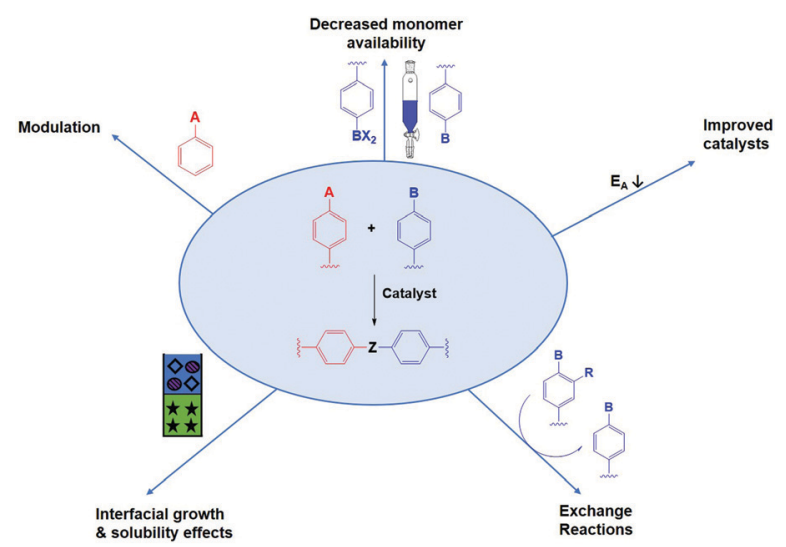

Fig. 8 Schematic representation of different approaches applied to improve COF quality. in Fig. 8) or in situ monomer generation (e.g. by linker protection, chemical approach). While the latter is more elegant, the former has already proven its value in the synthesis of $2 \mathrm{D}$ COF single crystals. ${ }^{104}$

Many synthetic protocols for COFs use catalysts to increase error-correction and speed up COF formation. Most common are solvothermal acetic acid catalyzed protocols, for example in the formation of imine COFs. However, some other techniques have been reported recently. These include the use of Ionic Liquids (IL), Deep Eutectic Solvents (DES), advanced acid catalysts and others. As those improved catalysts lower the activation energy for COF formation, reversibility is increased.

Interfacial growth and solubility effects can both improve COF quality by mitigating synthetic conditions. Due to reduced linker solubility or restriction of reaction to a specific interface, formation reactions are slowed down. Thus, less defects are formed resulting in high-quality thin films or powders.

Linker exchange can also be applied. Here, an initial (amorphous) network is formed, which is later transformed to a (more) crystalline COF by replacement of a linker by a new one. This way, new linkage types or previously inaccessible free functional groups can be obtained. As the COF is formed in a two-step process, conditions can be optimized to separate 'difficult to crystallize' steps from the crystallization.

Still, some other techniques remained undiscussed in this paragraph, most importantly linker and condition optimization. However, as these techniques are highly linker specific and can hardly be generalized, they are not the most attractive approaches to increase quality of the whole family of COFs. Still, some interesting effects and considerations are discussed in order to better understand COF chemistry.

Finally, there are some approaches that could not be assigned to one of the discussed categories. To be able to incorporate these, we created a category of other methods.

\subsection{Modulation in COF synthesis}

Modulation has been extensively studied in MOFs, offering among others - possibilities to enforce kinetic and particle size control, ultimately yielding improved crystallinity. ${ }^{149}$ However, while promising, it has been relatively underexplored for COFs with only very few examples available in literature.

3.1.1 Modulation in boronate ester COFs. The study of modulation in the synthesis of boronate ester COFs has been primarily focused on COF-5 (Fig. 9) with both a monofunctional boronic acid $^{151}$ and a monofunctional catechol tested as modulators. ${ }^{102,150}$ A first study, by Dichtel et al., was already shortly discussed in Section 2.1 and was part of an extended study on the kinetics of COF-5 formation. ${ }^{102}$ They used 4 -(tertbutyl)benzene-1,2-diol as a modulator (Fig. 9A) and observed a slower COF formation, resulting in an increased induction period as well as a slower rate of precipitation (Fig. 6B). The dependency on the modulator could be described by reverse first order kinetics. Moreover, the modulator was not incorporated in the framework, as evidenced by ${ }^{1} \mathrm{H}$ Nuclear Magnetic Resonance (NMR). Overall, it could be concluded that the modulator slows down the COF formation, but is not included 


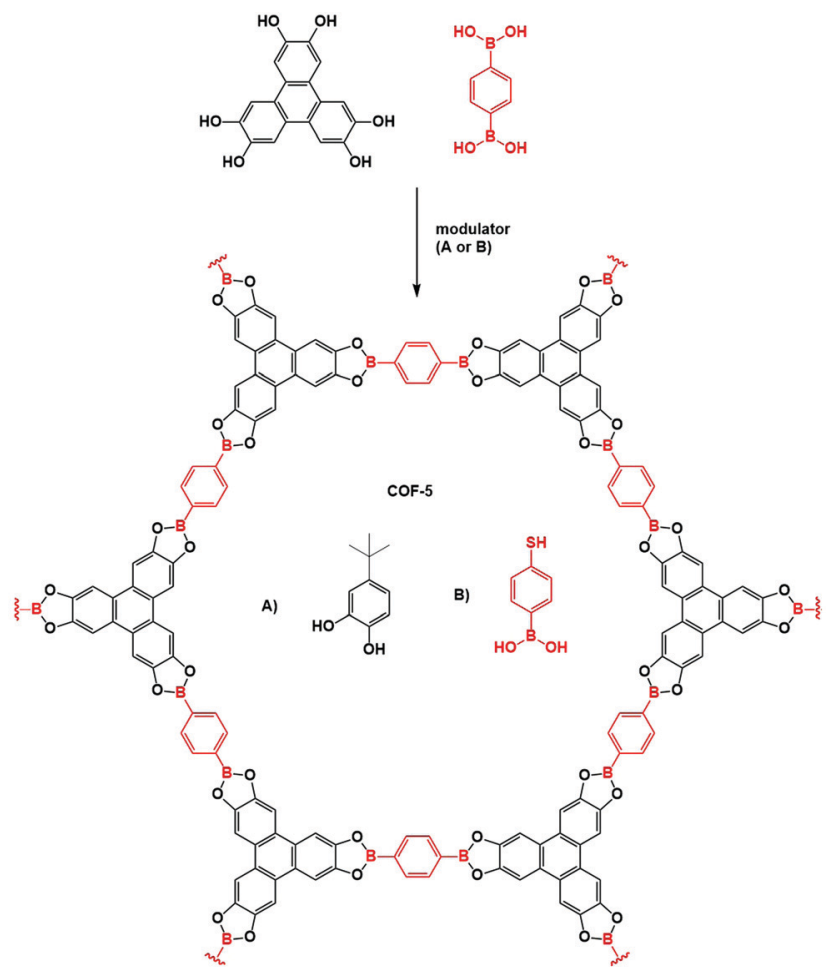

Fig. 9 Scheme of modulated COF-5 synthesis: 2,3,6,7,10,11-hexahydroxytriphenylene (black) and benzene-1,4-diboronic acid (red) are combined with a modulator to form COF-5. Used modulators are depicted in the pore: (A) 4-(tert-butyl)benzene-1,2-diol ${ }^{102,150}$ (B) 4-mercaptophenylboronic acid. ${ }^{151}$

in the irreversible precipitation, making this a promising tool to increase error-correction. In a more recent study, the same group used this modulator to chemically control COF growth from pre-synthesized colloids. ${ }^{150}$ Addition of 15 eq. of modulator could efficiently prevent nucleation, as evidenced by the lack of the (100) Bragg peak. Therefore, a monomer solution with 15 eq. of modulator was added to previously stabilized COF colloids (as discussed in Section 2.1). A significant increase in intensity and sharpening of the (100) WAXS peak was observed over two days. Furthermore, DLS measurements showed an increase of average particle size from 60 to $450 \mathrm{~nm}$ (for 4.5 eq. of monomers added), indicating the capability of modulators to enlarge COF crystals. An increase in BrunauerEmmett-Teller (BET) surface area from $1740 \mathrm{~m}^{2} \mathrm{~g}^{-1}$ to $1990 \mathrm{~m} \mathrm{~g}^{-1}$ close to the theoretical maximum was also observed, indicating high-quality crystalline COFs with accessible pores. Finally, the obtained results were extended to three other boronate ester COFs (TP-COF, DPB-COF and COF-10) with the largest obtained crystals of TP-COF (1400 nm via DLS) after $8 \mathrm{~h}$ of heating with 9 eq. of monomer and 15 eq. of modulator. Kinetic Monte Carlo simulations show that these effects are due to the limitation of formed oligomers below the critical nucleus size. Monoboronic acids have also been used in the synthesis of COF-5 (Fig. 9B). ${ }^{151}$ In this study, boronic acid monomers were systematically substituted for the 4-mercaptophenylboronic acid modulator, with the degree of substitution varying from 0 to $70 \%$. When small quantities of modulator were used (5-10\%), high crystallinity was confirmed via the observation of sharp and high-order PXRD reflections. Meanwhile, when higher amounts of modulator were added, quality of the crystallites gradually decreased with a remarkable drop at $70 \%$ of modulator used. Similar effects could be observed in $\mathrm{N}_{2}$-sorption experiments with an increase in BET surface area and pore volume, from $1200 \mathrm{~m}^{2} \mathrm{~g}^{-1}$ and $0.64 \mathrm{~cm}^{3} \mathrm{~g}^{-1}$, respectively, for an unmodulated COF-5 sample and to $2100 \mathrm{~m}^{2} \mathrm{~g}^{-1}$ and $1.14 \mathrm{~cm}^{3} \mathrm{~g}^{-1}$ for COF-5 synthesized with $10 \%$ modulator. A distinct step in the isotherm was obtained, indicating highly ordered and uniform pores. However, from $>25 \%$ of modulator, a broadened slope was observed due to the incorporation of modulator in the COF structures at these high modulator concentrations. Time-dependent PXRD experiments showed a slower COF crystallization when modulator was used. Only after $16 \mathrm{~h}$ of reaction time, the intensity of reflections for the modulated material catched up with the unmodulated COF, after which the modulated COF outperforms the unmodulated material. The crystallite sizes were estimated by Transmission Electron Microscopy (TEM) measurements, showing domains of several hundred of nanometers for the sample with $10 \%$ modulator (in contrast to $30-50 \mathrm{~nm}$ for the unmodulated sample). Finally, the effect of the functional group on the para position of the modulator was studied. When a carboxylic acid group was used instead of the thiol, obtained effects were highly similar. But when nonfunctionalized phenylboronic acid was used, no crystalline COFs were produced, most likely due to incompatible solubility and polarity.

3.1.2 Modulation in imine COFs. More is known about modulation in imine COFs, with the report of single crystal 3D COFs in 2018 as primary example. ${ }^{3}$ This was achieved by the addition of high quantities of aniline (Am1, Fig. 10) to the reaction mixture. As can be seen in Fig. 11A (mechanistically explained in Section 2.2), addition of sufficient amounts of Am1 as modulator creates an equilibrium resulting in slow $\mathrm{COF}$ precipitation, and the resulting morphologies can be compared to those observed for polycrystalline COFs in Fig. 11B. The main target of the study, COF-300, could be obtained as crystalline blocks of $100 \mu \mathrm{m}$ after 30-40 days of reaction at room temperature with 15 eq. of Am1. This allowed the structure solution up to $0.85 \AA$ A. To demonstrate the generality of their approach, the authors grew three more COFs to crystals suitable for SXRD: COF-303 (52 eq. of Am1, 15 days, $15 \mu \mathrm{m}$ crystals), LZU-79 (25 eq. of Am1, 50 days, $100 \mu \mathrm{m}$ crystals) and LZU-111 (80 eq. of Am1, 80 days, $60 \mu \mathrm{m}$ crystals). In a recent follow-up paper, ${ }^{122}$ a non-interpentrated 3D COF (of pts topology) was made via the same procedure, namely LZU-306 (the condensation product of adamantane-1,3,5,7-tetracarbaldehyde, ATA, and ETTA). Indeed, $50 \mu \mathrm{m}$ crystals could be obtained by 25 day reaction with 50 eq. of Am1. Still, crystal quality was not sufficient to allow direct structure elucidation via SXRD, extra RED experiments were necessary. Most likely this is a consequence of the highly porous non-interpenetrated structure (BET surface area $=2059 \mathrm{~m}^{2} \mathrm{~g}^{-1}$ and $80 \%$ void volume) allowing the inclusion of many disordered solvent molecules in 


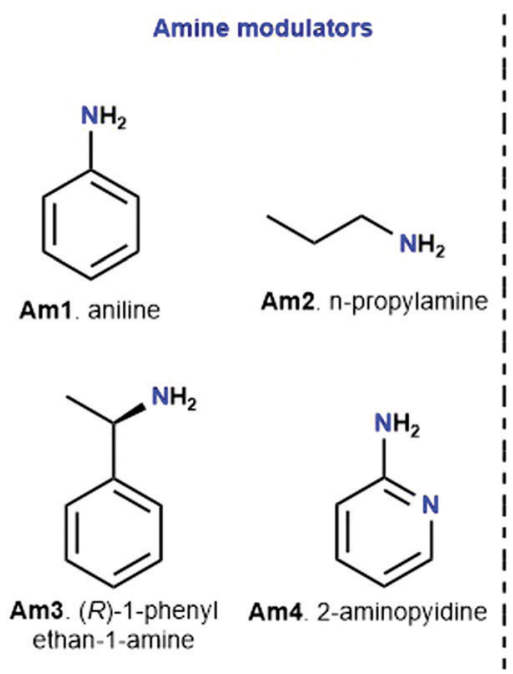

Aldehyde modulators<smiles>O=Cc1ccccc1</smiles>

Al1. benzaldehyde

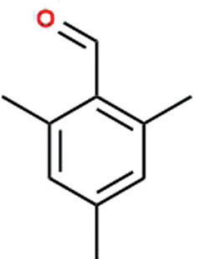

Al2. 2,4,6-trimethyl benzaldehyde

Fig. 10 Different modulators used in the synthesis of imine COFs, divided in amine modulators (blue) and aldehyde modulators (red): aniline (Am1) $n$-propylamine (Am2), (R)-1-phenylethan-1-amine (Am3) and 2-aminopyridin (Am4) were used as amine modulators, while benzaldehyde (Al1) and 2,4,6-trimethylbenzaldehyde (AI2) have been reported as aldehyde modulators.

A

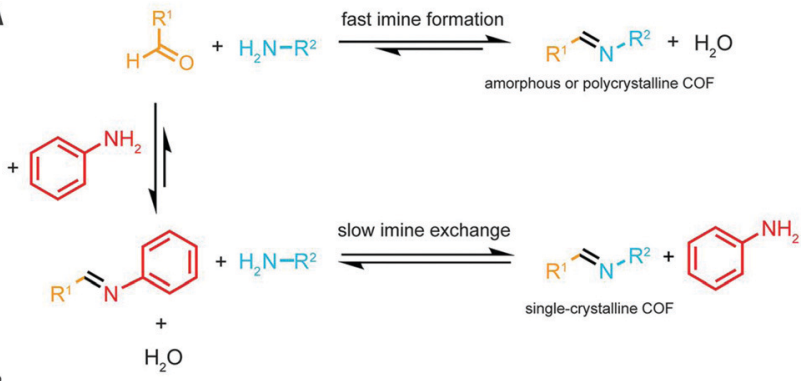

B
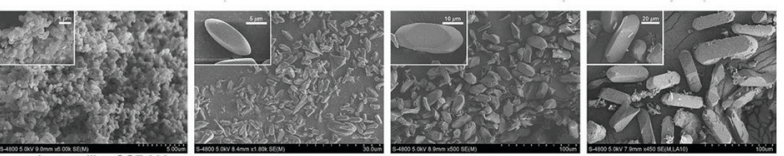

polycrystalline COF-300
(in the absence of aniline)

$\operatorname{COF}-300(\sim 10 \mu \mathrm{m})$

COF-300 $(\sim 20 \mu \mathrm{m})$ COF $300(\sim 60 \mu \mathrm{m})$
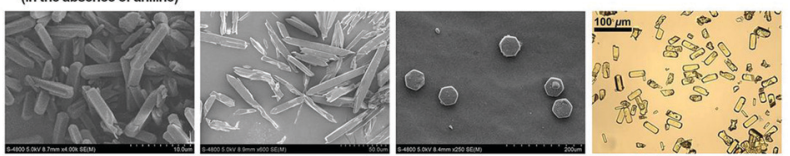

COF $303(-15 \mu \mathrm{m})$ LZU-79 ( $100 \mu \mathrm{m})$

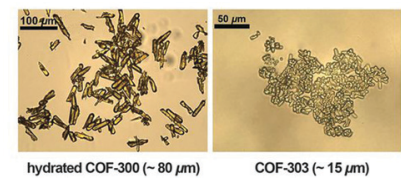

LZU-111 ( $50 \mu \mathrm{m})$ $\operatorname{COF}-300(-60 \mu \mathrm{m})$
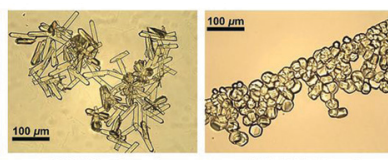

Fig. 11 (A) Schematic representation of COF synthesis with and without aniline addition. Without the added modulator, COF formation is favored, resulting in fast nucleation and limited growth and thus amorphous or polycrystalline COFs. When sufficient amounts of aniline are added, an equilibrium is obtained, allowing the growth of large COF single crystals via slow imine exchange. (B) SEM and optical microscopy images of singlecrystalline COFs. The SEM image of polycrystalline COF-300 is shown for comparison. Reproduced with permission from ref. 3, Copyright American Association for the Advancement of Science (2018)

Table 1 Influence of modulator concentration on crystal size of LZU-306

\begin{tabular}{lllllc}
\hline Entry & $\begin{array}{l}\text { Aniline } \\
(\text { eq. })\end{array}$ & $\begin{array}{l}\text { Acetic acid } \\
\text { solution }\end{array}$ & $\begin{array}{l}\text { Time } \\
(\text { days })\end{array}$ & $\begin{array}{l}\text { Temperature } \\
\left({ }^{\circ} \mathrm{C}\right)\end{array}$ & $\begin{array}{l}\text { Crystal } \\
\text { size }(\mu \mathrm{m})\end{array}$ \\
\hline 1 & 0 & $6 \mathrm{M}, 0.1 \mathrm{~mL}$ & 5 & 120 & 0.2 \\
2 & 7.5 & $6 \mathrm{M}, 0.1 \mathrm{~mL}$ & 5 & 120 & 0.5 \\
3 & 10 & $6 \mathrm{M}, 0.1 \mathrm{~mL}$ & 5 & 120 & 1 \\
4 & 12.5 & $6 \mathrm{M}, 0.1 \mathrm{~mL}$ & 5 & 120 & 5 \\
5 & 20 & $6 \mathrm{M}, 0.2 \mathrm{~mL}$ & 7 & 120 & 10 \\
6 & 40 & $6 \mathrm{M}, 0.2 \mathrm{~mL}$ & 25 & 30 & 20 \\
7 & 50 & $15 \mathrm{M}, 0.2 \mathrm{~mL}$ & 25 & 30 & 50
\end{tabular}

Used conditions: $9.9 \mathrm{~g}$ adamantane-1,3,5,7-tetracarbaldehyde $(0.04 \mathrm{mmol})$ in $0.5 \mathrm{~mL} 1,4$-dioxane + aniline + acetic acid solution. Separate addition of $15.7 \mathrm{mg}$ tetrakis(4-aminophenyl)ethylene) $(0.04 \mathrm{mmol})$ in $1 \mathrm{~mL}$ 1,4-dioxane. Reproduced with permission from ref. 122, Copyright John Wiley and Sons (2020).

the framework. A direct relationship between modulator concentration and crystal size could be obtained, as shown in Table 1 . Even at high temperature $\left(120{ }^{\circ} \mathrm{C}\right)$ and short times (five days) crystal size could be improved from $0.2 \mu \mathrm{m}$ (entry 1) to $5 \mu \mathrm{m}$ (entry 4). At higher concentrations of modulator (entries 6 and 7) more acid catalyst was required to grow crystals in a reasonable time. However, too much modulator was not beneficial, as no solids were obtained when more than 50 eq. of Am1 was used.

Meanwhile, experiments were performed with pre-modification of aldehyde monomers with the modulator, making in situ modulator generation via transimination possible. The synthesis of three different COFs (Fig. 12) by condensation of hydrazine hydrate with 1,3,5-triformylphenol (RIO-11), 1,3,5triformylresorcinol (RIO-12) or 1,3,5-triformylphloroglucinol (RIO-13, 1,3,5-triformylphloroglucinol = TFPM) was studied for gaining insight on the influence of modulator and H-bonding effect. ${ }^{152}$ Without modulator (Am1) addition, a clear increase in crystallinity and porosity with additional OH-groups in the aldehyde monomer could be observed. However, when using a modulated approach, even RIO-11 could be reticulated in a highly porous and crystalline COF. Using the two stepapproach (Fig. 12a) yielded a BET surface area of $1290 \mathrm{~m}^{2} \mathrm{~g}^{-1}$ and crystallite size of $64 \AA$, while the optimized one-step (Fig. 12b, 2 eq. of modulator) approach resulted in $1050 \mathrm{~m}^{2} \mathrm{~g}^{-1}$ for $60 \AA$ A crystallites (for comparison, an unmodulated synthesis of RIO-11 gave crystallites of $48 \AA$ with BET surface area of $\left.242 \mathrm{~m}^{2} \mathrm{~g}^{-1}\right)$. However, when applying the same methodology to RIO-12 and RIO-13, no improvement (RIO-12) or even a decrease (RIO-13) in materials quality was observed, most likely resulting from the irreversible keto-imine adduct formation. Another study combined the aforementioned TFPM with benzidine to form TFPM-BZ COF via a two-step transimination (as illustrated in Fig. 12A) with different amines. ${ }^{153}$ Highly crystalline COFs could be synthesized at $180{ }^{\circ} \mathrm{C}$ and ambient pressure in only $6 \mathrm{~h}$. BET surface areas of $1052.3 \mathrm{~m}^{2} \mathrm{~g}^{-1}$ (2-aminopyridin, Am4) and $1056.6 \mathrm{~m}^{2} \mathrm{~g}^{-1}$ (n-propylamine, Am2) could be obtained, much higher than the $537 \mathrm{~m}^{2} \mathrm{~g}^{-1}$ obtained by traditional solvothermal methods. Other modulators ( $n$-butylamine, isobutylamine, $n$-hexylamine and dodecylamine) were also tested and all resulted in highly crystalline COFs. Albeit, it seemed that mono-amines with high boiling points gave the sharpest PXRD peaks. Finally, the 


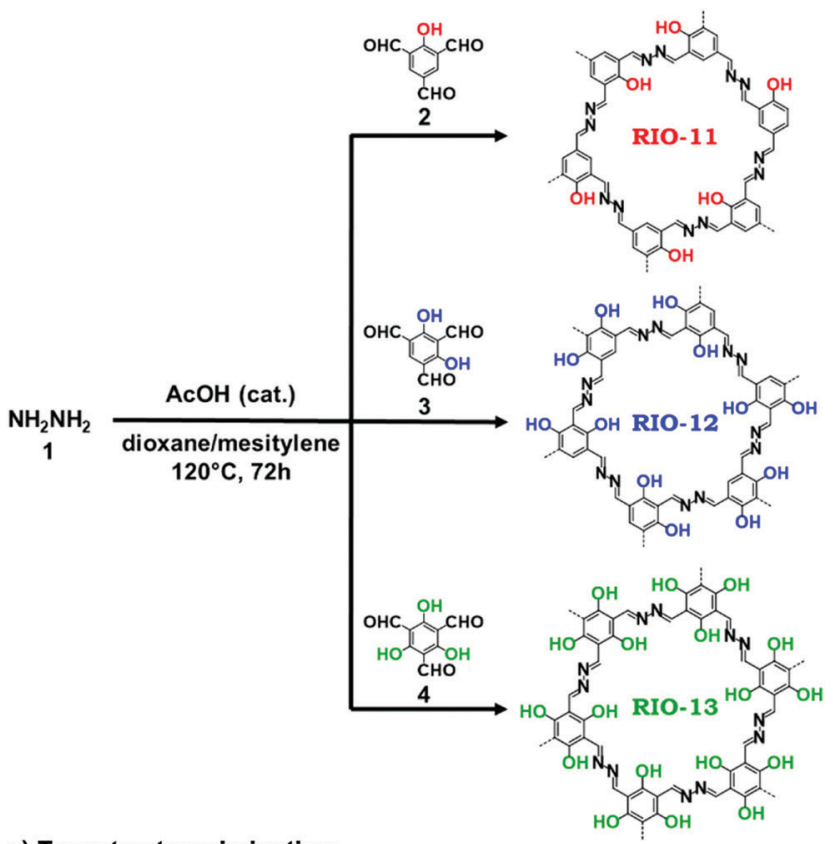

a) Two step transimination:

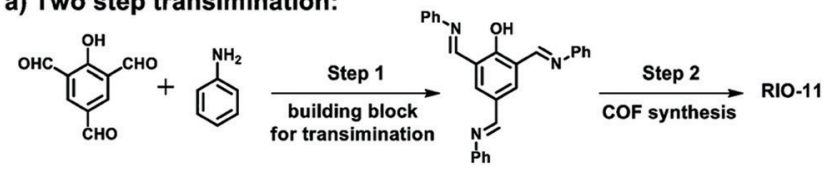

b) In situ transimination:

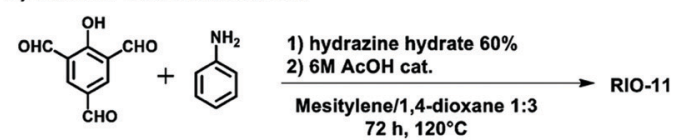

Fig. 12 Synthetic scheme for RIO COFs (RIO-11, RIO-12 and RIO-13). Two different modulation approaches are shown: (a) two step transimination. (b) In situ transimination. Reproduced with permission from ref. 152, Copyright American Chemical Society (2018).

approach was generalized to DHNDCA-TAPP COF (2,6-dihydroxynaphthalene-1,5-dicarbaldehyde, DHNDCA + 2,4,6-tris(4aminophenyl)-pyridine, TAPP) which could not be synthesized via traditional methods.

Aldehydes can also be used as modulators, as has been shown for TAPB-BDA COF where the addition of Al1 was studied. ${ }^{155}$ A sharpening and increase of intensity of the (100) diffraction peak, as well as a decrease in FWHM, was observed on increasing modulator concentration, indicating an increase in crystallinity. $\mathrm{N}_{2}$-Sorption experiments confirm these results, as an increase in BET surface area from $1378 \mathrm{~m}^{2} \mathrm{~g}^{-1}$ (for unmodulated COF) to $2480 \mathrm{~m}^{2} \mathrm{~g}^{-1}$ (for 9 eq. of benzaldehyde), indicating a more ordered porous structure. A second COF, TAPB-TFPA (TFPA: tris(4-formylphenyl)amine) confirmed the generality of the approach as 4 eq. of modulator caused a threefold increase in both (100) peak intensity as BET surface area. However, no significant difference in crystallite size and morphology was observed for the modulated materials, implying that benzaldehyde modulators might not be the best choice for growing large $\mathrm{COF}$ crystals. One report studied the combined use of an aldehyde and amine modulator, namely Al1 and Am1 for
TAPB-BTCA COF (BTCA: 1,3,5-benzenetricarbaldehyde) as shown in Fig. $13 .{ }^{154} 12$ eq. of both modulators were used in a Sc(OTf $)_{3}$ catalyzed synthesis. While unmodulated samples formed irreversible polycrystalline aggregates, modulation allowed selective production of spheres, hollow fibres and films, depending on monomer concentration, solvent composition and supporting bases. Moreover, sharper and more intense PXRD peaks were observed and BET surface area increased from $432 \mathrm{~m}^{2} \mathrm{~g}^{-1}$ for the unmodulated material to $882 \mathrm{~m}^{2} \mathrm{~g}^{-1}$ for the modulated material. Crystallinity could be even further improved by increasing the amount of acid catalyst. Moreover, the crystallite size of $17.6 \mathrm{~nm}$ was significantly higher than the $10.7 \mathrm{~nm}$ obtained for the unmodulated material (via Scherrer analysis). Studies on the relative ratio of the PXRD reflections showed a critical amount of 12 eq. of monomer, as going from 0 to 12 eq. continuously enhanced intensity and sharpness of the (100) reflection, but sample yield declined dramatically when more than 12 eq. of modulator were used. The obtained results could be extended to different catalysts (acetic acid), aldehyde monomer (BDA) and amine monomer ( $p$-phenylenediamine, PPD). A kinetic pathway was proposed, where, unmodulated COFs rapidly precipitate into amorphous polymers (kinetic traps, Fig. 13A) and then partially transform to thermodynamically favored crystalline materials (equilibrium state, Fig. 13A). When modulators are added, four different reactions can happen to any intermediate oligomer. A modulator can react with the oligomer to form IS-1 or IS-3 or it can react with one of the monomers to form IS-2 or IS-4. IS-1 can be converted to IS-2 due to the Schiff base reversibility, just as IS-3 and IS-4. As the latter (IS-2 and IS-4) can form crystalline COFs, they are thermodynamically favored, but both modulators are required to enhance the reversibility of all steps.

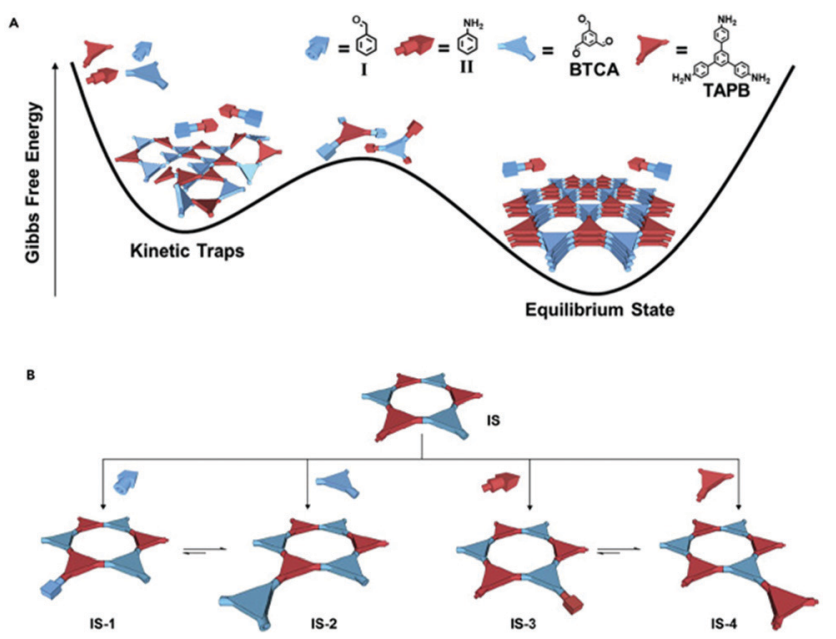

Fig. 13 Modulated synthesis of TAPB-BTCA COF in a cartoon representation. Aldehydes are shown in blue (rod: benzaldehyde, triangle: 1,3,5benzenetricarbaldehyde), amines in red (rod: aniline, triangle: 1,3,5-tris(4aminophenyl) benzene. (A) Kinetic traps and equilibrium state in function of Gibbs free energy. (B) Different reactions possible and the formed structures. Reproduced with permission from ref. 154, Copyright Elsevier (2019). 
Structures could also be modified by modulator addition. For example, chiral TFPM-TAPB COF could be synthesized from achiral linkers (TFPM and TAPB) by addition of a chiral modulator (Am3). ${ }^{156}$ In another study, different mesocrystalline structures were formed by adjustment of the modulator (between $n$-propylamine, $n$-butylamine, $n$-hexylamine, $n$-octylamine, $n$-dodecylamine and aniline), while unmodulated synthesis only formed irregular aggregates. ${ }^{157}$ Moreover, by slight alteration of reaction conditions, complicated mesostructures could be formed. Finally, ultrathin nanosheets could be formed by the addition of 40 eq. of Al2 ${ }^{158}$ Even though the sheets are amorphous on PXRD (most likely due to the disordered stacking of the nanosheets), Selected Area Electron Diffraction (SAED) patterns were sharp and matching with the patterns observed for exfoliated "traditional" COFs and a periodic well-defined lattice (matching with the theoretical lattice) was observed in Scanning Tunneling Microscopy (STM). The technique was generalized for four different COFs and also works with bromo-2,6dimethylbenzaldehyde as modulator. However, the use of 3,5-di-tert-butylbenzaldehyde, 2,6-dimethoxybenzaldehyde, and 3,5-bis(trifluoromethyl)benzaldehyde did not afford solid products, most likely because the substituted groups on the modulator impede nucleophilic attack on the intermediate compound.

3.1.3 Modulation and other linkage types. To the best of our knowledge, only one example exists of modulation in a non imine or boronate linked COF. Here, JCU-520, JCU-521, JCU-522 and JCU-523 are formed via reversible Michael addition-elimination of $\beta$-ketoenamines and aromatic amines (Fig. 14). ${ }^{159}$ The modulator, dimethylamine is formed in situ as a sideproduct, in contrast to the first report for this type of COF, which used $\beta$-ketoenols and thus formed $\mathrm{H}_{2} \mathrm{O}$ as a sideproduct. $^{61}$ This allowed the COFs to be formed at room temperature in eight hours, in contrary to the previously reported $130{ }^{\circ} \mathrm{C}$ and three days. ${ }^{159}$ Moreover, the compounds were found to be highly crystalline with sharp and intense PXRD reflections as well as highly porous with BET surface areas between 976 and $1435 \mathrm{~m}^{2} \mathrm{~g}^{-1}$. As the formed COFs are (slightly) different from previously obtained frameworks these values are hard to compare, but these values are significantly higher than the $505 \mathrm{~m}^{2} \mathrm{~g}^{-1}$ which was the maximal BET surface area obtained in the first report. ${ }^{61}$

\subsection{Decreasing monomer availability}

3.2.1 In situ linker generation. One elegant way to decrease the monomer availability is in situ linker generation from protected monomers. Different possibilities have been reported and are presented in Fig. 15. While not all of them formally lead to decreased monomer availability, all of them have their advantages in the synthesis of highly crystalline COFs.

The first example of a protecting group used is the acetonide catechol. ${ }^{160}$ This method has been developed because of undesirable features in the polyfunctional catechols used in boronate ester COF synthesis, which are hard to dissolve and prone to oxidation. Contrarily, the acetonides have decreased polarity, show no auto-oxidation and have enhanced solubility. Efficient boronate COF formation from acetonides could be achieved by

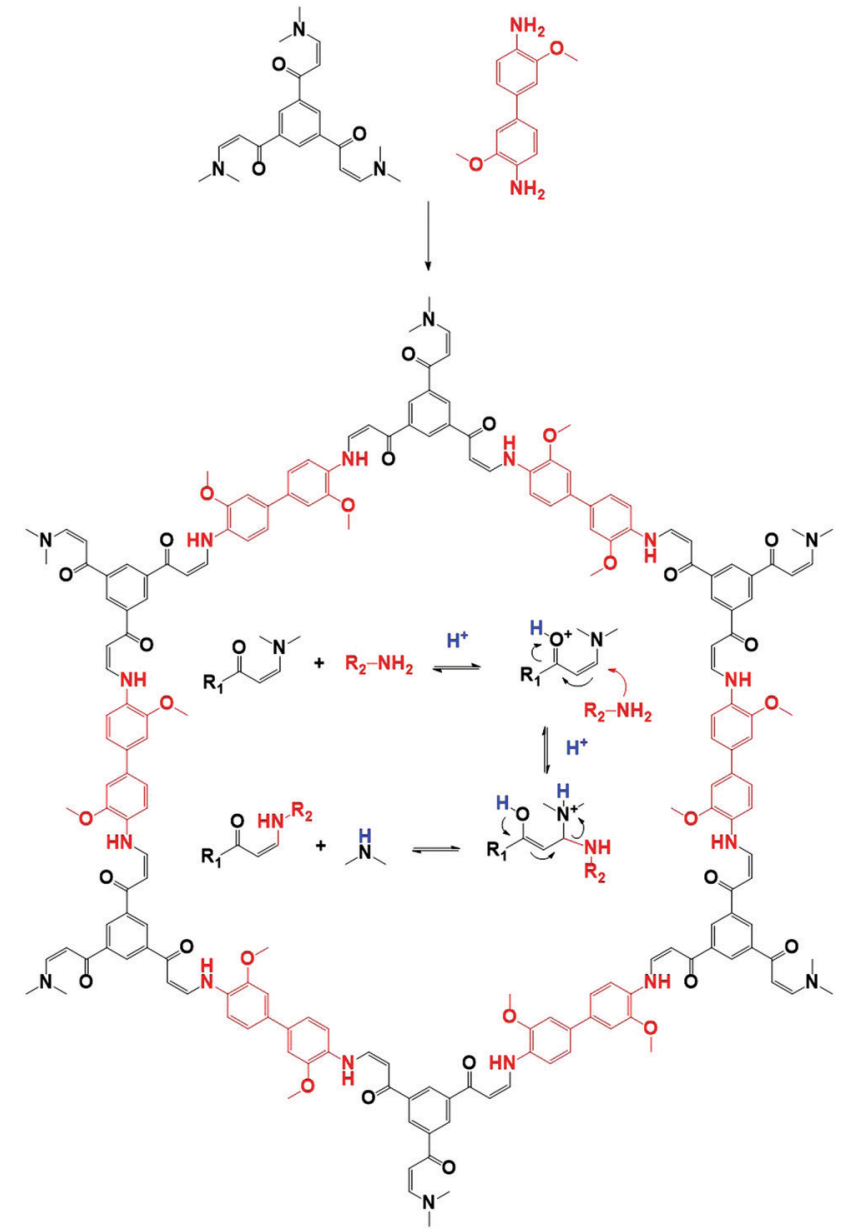

Fig. 14 Structural representation of a COF $(J \cup C-523)^{159}$ synthesized by Michael addition-elimination of a $\beta$-ketoenamine and an amine. The mechanistic pathway is shown in the pore.

$\mathrm{BF}_{3}$. OEt ${ }_{2}$ catalysis. Three COFs (COF-5, COF-10 and NiPc-PBBA $\mathrm{COF}$ ) prepared through this method have been compared to materials synthesized via direct condensation. ${ }^{161}$ No significant difference in quality between both methods could be found for COF-5 and COF-10 with sharp PXRD reflections and high porosity for all materials. However, for NiPc-PBBA COF, a modest improved in quality was observed, as shown by sharper PXRD reflections and a slight increase in surface area (776 $\mathrm{m}^{2} \mathrm{~g}^{-1}$ vs. 624 for the direct condensation). It has to be noted that high catalyst loadings were necessary to synthesize these materials, which was attributed to complex formation between free boronic acids and $\mathrm{BF}_{3} \cdot \mathrm{OEt}_{2}$. Still, as most boronic acids immediately rearrange to boroxines, this side reaction is not too problematic. Boronate ester COFs have also been reported from dioxaborolane protected boronic acids, which have improved solubility. ${ }^{162}$ Subsequent reaction was done in two steps under microwave addition. In a first step the dioxaborolane was heated with $\mathrm{HCl}$ for 10 minutes. Afterwards the catechol was added and the COF was left to form over 30 minutes. While high porosity and sharp PXRD reflections were observed using this method, one-pot deprotection turned out to be challenging. 

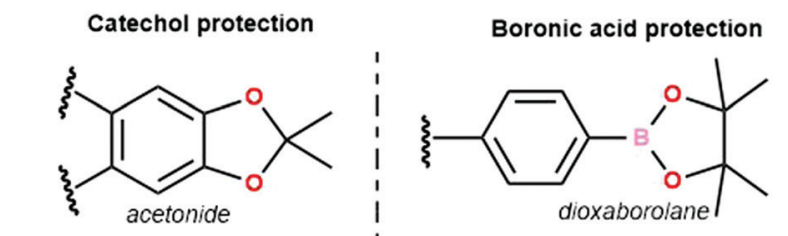

$----------$
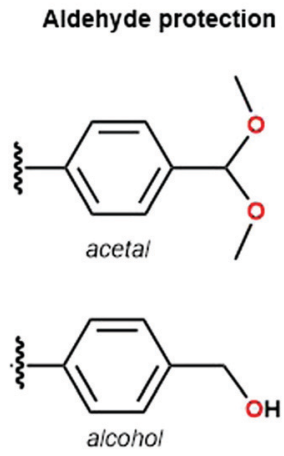

i

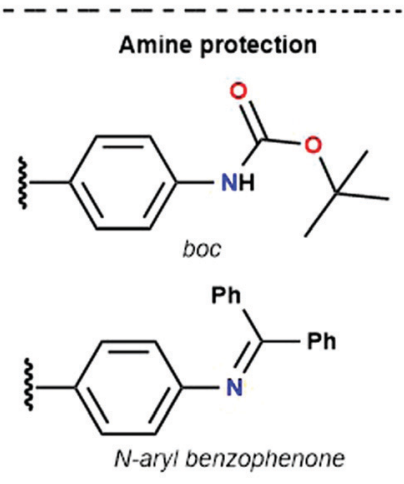

Fig. 15 Various protection groups used in the synthesis of COFs. Catechol monomers have been protected as acetonides, boronic acids as dioxaborolanes, aldehydes as acetals and alcohols, and amines as $\mathrm{N}$-arylbenzophenones and boc (tert-butyloxycarbonyl) groups.

Aldehydes used for COF formation have been protected as acetals $^{163}$ and in situ generated by the oxidation of alcohols. ${ }^{164}$ The former has been proven to work for imine, azine and hydrazone COFs, while the latter was used in the synthesis of Crystalline Triazine Frameworks (CTFs). Advantages of acetal protection are enhanced solubility and stability. Also, Electrospray Ionization Mass Spectrometry (ESI-MS) measurements did not find aldehyde in the reaction mixture, indicating direct condensation of amine and acetal. ${ }^{163}$ Highly crystalline and porous LZU-20, LZU-21 and LZU-22 were synthesized using this method. Alcohols could be used for synthesis of CTF-HUST-C1, CTF-HUST-C5, CTF-HUST-C6 via slow oxidation in DMSO. ${ }^{164}$ Using FTIR measurements at different times in the reaction, it could be noticed that characteristic triazine peaks emerged as alcohol peaks disappeared, proving that the alcohol oxidation was the rate limiting step. It was also noted that careful temperature control was needed to obtain high-quality material, initial low temperatures ensured slow initial nucleation, subsequent higher temperatures provided faster growth. Three different crystalline CTFs were obtained using this method, which is quite remarkable given the enormous difficulty in obtaining crystalline CTFs. ${ }^{141}$

The tert-butyloxycarbonyl (boc) group is one of the most widely utilized amine protection groups. ${ }^{165}$ Therefore, it is no surprise that it has found use in the synthesis of COFs. ${ }^{83}$ In the synthesis of the woven COF-112, boc protection is used to guide the reaction via the "right" pathway, as shown in Fig. 16. Initial rapid imine condensation forms NHBoc-DIP, but cannot continue to an amorphous polymer because the second amine function is boc protected. This enables the coordination of $\mathrm{Co}^{2+}$ and formation of the $\mathrm{Co}(\mathrm{NHBoc}-\mathrm{DIP})_{2}\left(\mathrm{BF}_{4}\right)_{2}$ complex which already has the desired symmetry. Finally, the in situ

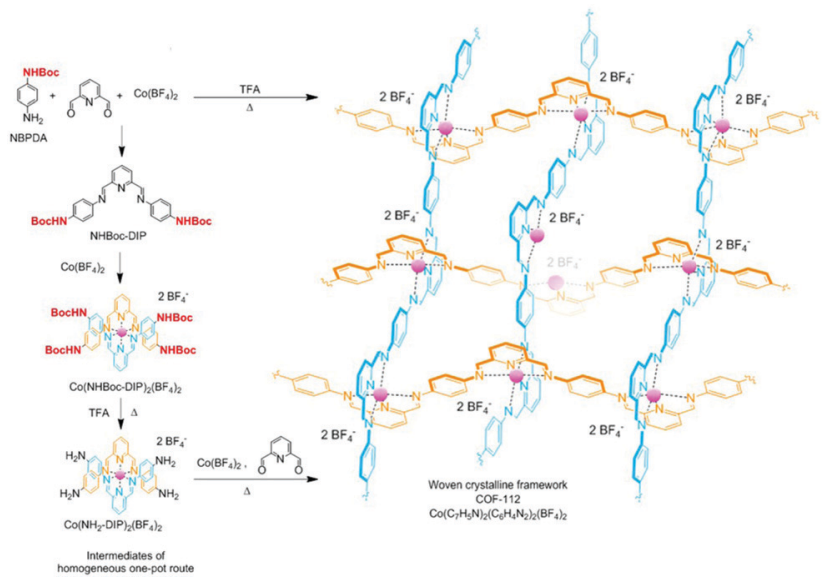

Fig. 16 Synthetic scheme for the synthesis of COF-112 via boc protected amines. Adapted and reproduced with permission from ref. 83, Copyright American Chemical Society (2017).

deprotection of the amines by trifluoroacetic acid can react with the remaining aldehydes to form the crystalline COF. COF-112 could also be made from previously synthesized $\mathrm{Co}\left(\mathrm{NH}_{2} \text {-DIP }\right)_{2}\left(\mathrm{BF}_{4}\right)_{2}$ but the boc protected group gave larger crystallites which could be analyzed by 3D-EDT. The method has also been extended to an isostructural iron based wCOF (COF-112-Fe) and three imine COFs (LZU-1, TFPB-BDA and Por-COF). The imine COFs could be obtained as highly uniform nanocrystals using microwave synthesis in ethanol. LZU-1 nanoparticles of $245 \pm 25 \mathrm{~nm}$ (SEM) could be obtained with clearly visible pores (HR-TEM). Moreover, a BET surface area of $729 \mathrm{~m}^{2} \mathrm{~g}^{-1}$ was obtained (while the original report of LZU-1 gave $457 \mathrm{~m}^{2} \mathrm{~g}^{-1}$ ) indicating the high crystallinity of this COF. Finally, $N$-aryl benzophenones, which are common intermediates in the synthesis of aryl amines, could also be used in COF synthesis. ${ }^{166}$ Advantages of this protection include enhanced solubility, less danger of oxidation and easier purification than what is observed for amines. BND-TFB COF ( $N$-benzidine benzophenone + BTCA) was prepared using microwave irradiation and traditional methods; results for different methods are summarized in Table 2. Comparing direct reaction (entry 10) to protected amine (entry 2), a clear difference (1501 and $2314 \mathrm{~m}^{2} \mathrm{~g}^{-1}$, respectively) is observed, showing the improved quality of the material synthesized from the protected monomer. Additionally, good quality (entry 1, $1938 \mathrm{~m}^{2} \mathrm{~g}^{-1}$ ) is already obtained after $24 \mathrm{~h}$ and when left for five days the quality improves even further (entry $3,2618 \mathrm{~m}^{2} \mathrm{~g}^{-1}$ ). Microwave irradiation made it possible to obtain high-quality COF in seven hours (entry 9, $2200 \mathrm{~m}^{2} \mathrm{~g}^{-1}$ ) and moderate quality in as fast as 1 hour $\left(629 \mathrm{~m}^{2} \mathrm{~g}^{-1}\right)$. Moreover both vacuum activation (entry 4, $2313 \mathrm{~m}^{2} \mathrm{~g}^{-1}$ ) and reaction upscaling (entry $6,2321 \mathrm{~m}^{2} \mathrm{~g}^{-1}$ ) do not cause any problems. Finally, oxidation resistance of the benzophenone was confirmed using reactions under air and under vacuum (entries 5 and 7). As air environment did not cause significant differences in quality $\left(2327 \mathrm{~m}^{2} \mathrm{~g}^{-1}\right)$ and vacuum even a diminishment $\left(1677 \mathrm{~m}^{2} \mathrm{~g}^{-1}\right)$, it was concluded that all reagents and intermediates were stable to oxidative degradation. 
Table 2 Different synthetic conditions for BND-TFB COF and obtained surface areas

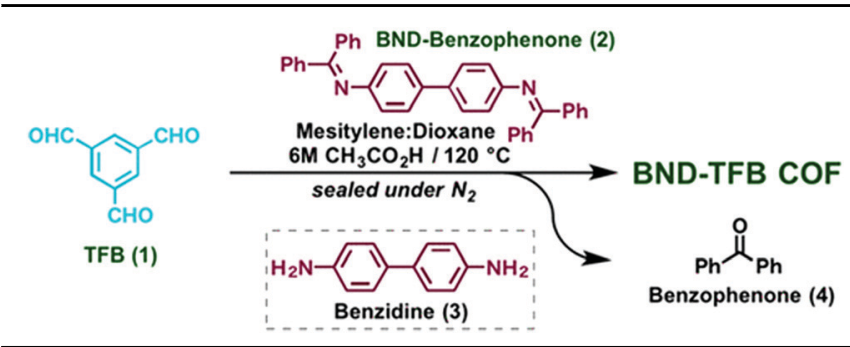

\begin{tabular}{llll}
\hline Entry & Deviation from conditions & Time & $S_{\text {BET }}\left(\mathrm{m}^{2} \mathrm{~g}^{-1}\right)$ \\
\hline 1 & - & 24 (h) & 1938 \\
2 & - & 3 (days) & 2314 \\
3 & - & 5 (days) & 2618 \\
4 & Vacuum activation & 3 (days) & 2313 \\
5 & Under air & 3 (days) & 2327 \\
6 & 15 $\times$, vacuum activation & 3 (days) & 2321 \\
7 & Under vacuum & 3 (days) & 1677 \\
8 & Microwave, under air & 1 (h) & 629 \\
9 & Microwave, under air & 7 (h) & 2200 \\
10 & 3 used instead of 2 & 3 (days) & 1501 \\
11 & 3 used with 2 eq. of 4 & 3 (days) & 1453
\end{tabular}

Activation was done using supercritical $\mathrm{CO}_{2}$ unless otherwise specified. Reproduced with permission from ref. 166, Copyright American Chemical Society (2017).

Finally, any modulating capabilities of the generated benzophenone were ruled out as separate addition of this did not change the quality of the synthesized COF (entry $11,1453 \mathrm{~m}^{2} \mathrm{~g}^{-1}$ ). The method was generalized to three other COFs (TPPA-1, TFPM-BZ COF, TAPB-BDA COF), which showed a slightly improved surface area and sharp PXRD reflections when the $\mathrm{N}$-aryl benzophenone was used.

3.2.2 Slow linker addition. The most straightforward way to decrease the amount of available linkers in the reaction mixture is slow linker addition. This technique could for example be used in the synthesis of oriented thin films of $\beta$-ketoenamine COFs. ${ }^{167}$ While crystalline DAAQ-TFP COF powder can directly be obtained from condensation of TFPM and 2,6-diaminoanthraquinone, this was not the case for thin films. However, when a solution of the aldehyde linker in DMF was added slowly (over the course of 1 hour) to a DMF solution of the amine linker, crystalline, oriented films were obtained as observed by Grazing Incidence X-ray Diffraction (GID). Moreover, a difference in film thickness in relation to the initial monomer concentration was observed. Thicker film consecutively led to more intense diffraction. However, the origin of this effect could not be distinguished. Slow linker addition could also be used in CTF synthesis, as an alternative to the in situ alcohol oxidation described in Section 3.2.1. ${ }^{168}$ Different feeding rates were studied for their influence on FWHM of the main PXRD reflection. When lowering the feeding rate from 800 to $30 \mu \mathrm{L} \mathrm{min}{ }^{-1}$ the FWHM decreased from 2.20 to $1.82^{\circ}$. However, when the feeding rate was decreased even more to $10 \mu \mathrm{L} \mathrm{min} \operatorname{mos}^{-1}$ a loss of smoothness in Scanning Electron Microscopy (SEM) images was observed, indicating limitations to the method. Finally, temperature is also an important factor

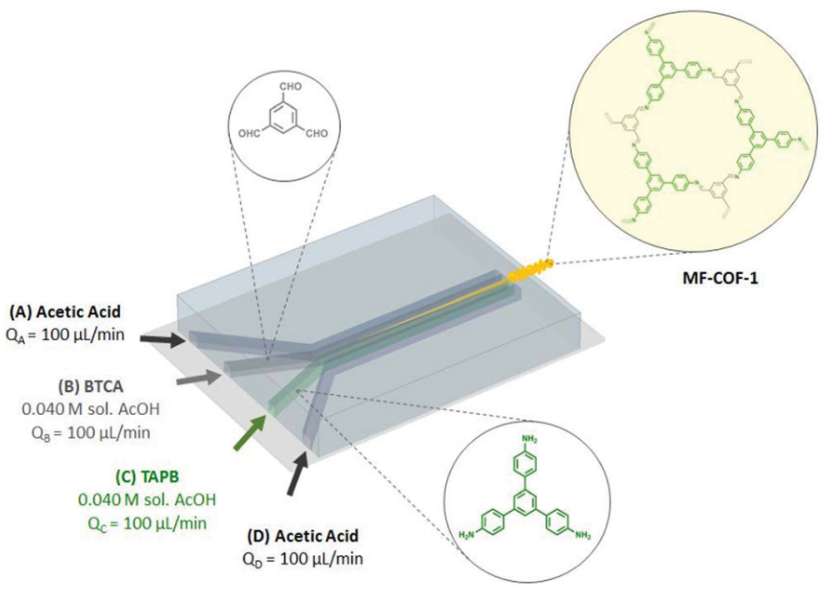

Fig. 17 Schematic representation of microfluidic COF synthesis. Adapted and reproduced with permission from ref. 169, Copyright Royal Society of Chemistry (2016).

and it was found that initial temperature of $100{ }^{\circ} \mathrm{C}$ yielded the best FWHM. Most likely, lower temperatures do not provide enough energy to efficiently form the triazine rings, while higher temperatures induce more nucleation. The slow addition technique could also be automated with a homemade microfluidic device. ${ }^{169}$ The monomers can be added via different channels and mixed in the main chamber, a process which is illustrated in Fig. 17. By careful control of the flow rate, synthesis of TABP-BTCA COF could be diffusion controlled and the chamber did not become clogged with formed COF. To improve reaction rate and reversibility, pure acetic acid was used as solvent, which allowed the COF formation to take place in a few seconds. Remarkably, the resulting material showed high crystallinity with lower peak widths than its traditional counterpart, but surface area was slightly lower. SEM images showed $40 \mathrm{~nm}$ particles linearly arranged into fibres, which could be used in 3D printing of the material.

As described in Section 2.1, slow monomer addition has been used for the synthesis of large single crystals of $2 \mathrm{D}$ COFs. ${ }^{104}$ First, stable COF-5 colloids of $400 \mathrm{~nm}$ were formed in ACN. Subsequently, this suspension was heated to $85{ }^{\circ} \mathrm{C}$ and linker solutions were added simultaneously. When monomers were added slowly ( 0.1 eq. per $h)$, the average crystal size gradually increased up to $1 \mu \mathrm{m}$ (Fig. 6E). However, when the rate of linker addition was increased to 1.0 eq. per $\mathrm{h}$ the particle size gradually decreased to finally settle around $50 \mathrm{~nm}$. Most likely, the concentration barrier for nucleation was crossed in the fast addition growing regime. The critical monomer concentration could be estimated around $1 \mathrm{mM}$ by the diversion point in Fig. 6E. WAXS measurements (Fig. 6F) confirmed the increased crystallinity of the particles during slow addition, as PXRD reflections intensified and sharpened, indeed particles seemed single-crystalline on HR-TEM. The method was also generalized to two other boronate ester COFs (COF-10, up to $190 \mathrm{~nm}$ and TP-COF, up to $750 \mathrm{~nm}$ ). However, due to aggregation, the single crystalline nature of TP-COF could not be confirmed via HR-TEM. In 2019, the method was extended to 
2D imine COFs. ${ }^{170}$ Crystalline colloids of three different imine COFs were stabilized in ACN solutions. Sizes and crystallinity could be controlled by the initial monomer concentration, with higher concentration resulting in increased particle size and crystallinity (TAPB-BDA COF for example could reach $330 \mathrm{~nm}$ at initial TAPB concentration of $4.25 \mathrm{mM}$ ). This was attributed to the typical re-crystallization of amorphous polymer processes in imine COFs, which is more efficient when larger amounts of linkers are present. Subsequent monomer addition afforded particles of up to $690 \mathrm{~nm}$ for an adding rate of 1 eq. per $\mathrm{h}$. Surprisingly, when monomers were added more rapidly (50 eq. per h), particle size increased initially (up to two added eq.) to $490 \mathrm{~nm}$. Only when more monomers were added, particle size started to decrease (up to $230 \mathrm{~nm}$ for $6 \mathrm{eq}$.) due to the nucleation of new crystallites. However, no associated change in WAXS (100) diffraction peak FHWM with particle growth was observed, indicating that the domain size was left unchanged. Most likely the newly added monomers form an amorphous shell around the colloid, which slowly recrystallizes over time.

\subsection{Improved catalysts}

3.3.1 Ionic liquids and deep eutectic solvents. IL and DES are emerging green solvents. Due to the high amounts of ions present and the low vapor pressure, these solvents can be very attractive for synthesis of crystalline materials. Indeed, multiple examples of IL and DES mediated MOF synthetic approaches exist. ${ }^{171}$ However, in COFs only limited studies have been done on IL and DES based synthetic methods. The first report of IL use for the synthesis of COFs, by Guan et al. was published as recently as 2018. ${ }^{172}$ They synthesized three different 3D imine COFs in [BMim] $\left[\mathrm{NTf}_{2}\right]$ (Fig. 18A) and obtained highly crystalline materials in 12 hours, at room temperature. As little as $100 \mu \mathrm{L}$ [BMim $]\left[\mathrm{NTf}_{2}\right]$ was enough to act as both solvent and catalyst and could be easily separated and reused by filtration without inducing activity loss. This report gave rise to several follow-up studies on the use of IL in COF synthesis. For example, TFPPyBDA COF and TFPPy-PyTTA COF were synthesized in [BMIm] with different anions $\left(\mathrm{Br}^{-}\right.$, OTf ${ }^{-}, \mathrm{p}^{-} \mathrm{Ts}^{-}, \mathrm{CF}_{3} \mathrm{CO}_{2}{ }^{-}, \mathrm{N}(\mathrm{CN})_{2}{ }^{-}$, $\mathrm{NO}_{3}{ }^{-}, \mathrm{SCN}^{-}$, and $\mathrm{NTf}_{2}{ }^{-}$) with added acetic acid (3 d, $\left.120{ }^{\circ} \mathrm{C}\right) .{ }^{173}$ However, only the previously used [BMim] $\left[\mathrm{NTf}_{2}\right]$ yielded crystalline products. The formed materials were compared to solvothermally synthesized analogues and proven to be superior. Higher order PXRD reflections were observed and SEM showed more uniform and larger crystallites. The method could also be extended to azine linked 3D-HNU-5 ${ }^{174}$ and five different $\beta$-ketoenamine COFs. ${ }^{175}$ In this final example, $[\mathrm{BMim}]\left[\mathrm{BF}_{4}\right]$ was used as ionic liquid, at $50{ }^{\circ} \mathrm{C}$, causing precipitation of highly crystalline COF in 12 hours. The authors noted that, while a crystalline COF could also be formed in a mixture of organic solvent (DMSO) and IL, pristine organic solvent only yielded amorphous material, showing the catalytic potential of the IL. PXRD analysis revealed higher order reflections in the IL sample than its traditional counterpart, indicating increased crystallinity. Surprisingly, some mesoporosity was observed in the $\mathrm{N}_{2}$-sorption isotherm of the IL mediated sample, which was not present in the traditional material. Subsequent experiments
A)

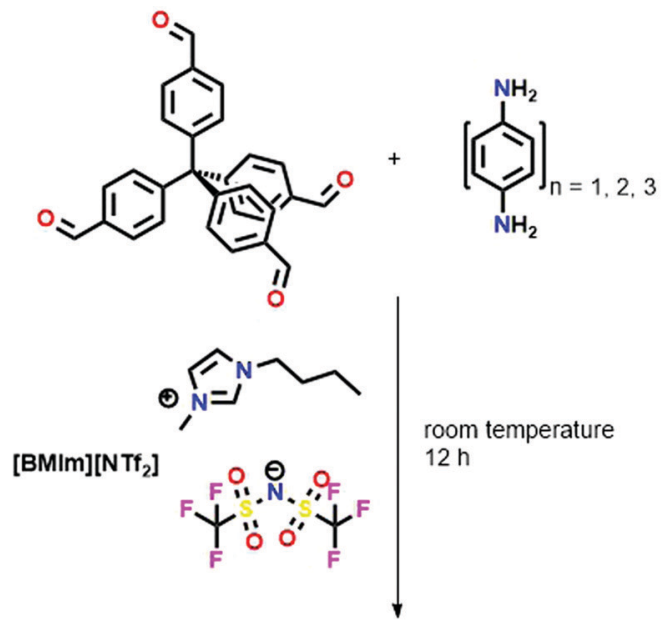

Crystalline COF

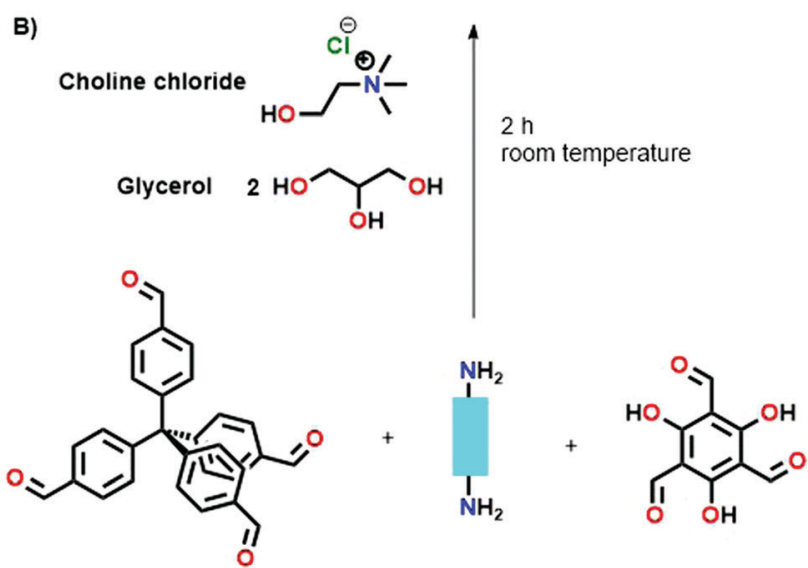

Fig. 18 (A) Schematic representation of COF synthesis via ionic liquids. Here, $[B M I m]\left[N \mathrm{Nf}_{2}\right]$ is used to link TFPM and PDA. (B) Schematic representation of COF synthesis in Deep Eutectic Solvents. Choline chloride/ glycerol (1/2) is used to link different aromatic amines with TFPM or TAM.

with different ILs (where the alkyl side chain of the cation was replaced from butyl to hexyl, HMim, decyl, DMim, or dodecyl, DoMim) revealed a decrease in crystallinity and even amorphous material when DoMim was used. Interestingly, the pore diameter of the mesopores seemed to depend on the used IL, with a maximal pore diameter of $25 \mathrm{~nm}$ for DMim. Most likely this results from IL templating in the pore, made possible by IL-amine H-bonds and cation- $\pi$ interactions. ${ }^{173,175}$ Very recently, DES have also been reported for the synthesis of COFs. ${ }^{176}$ Different $2 \mathrm{D}$ and $3 \mathrm{D}$ COFs were synthesized in a $1 / 2$ mixture of choline chloride and glycerol (ChCl/Gly) (Fig. 18B), affording highly crystalline compounds after two hours at room temperature. The DES could be reused at least three times without activity loss and the synthesis could be scaled up to $1.6 \mathrm{~g}$ scale. $\beta$-Ketoenamine, azine and hydrazone 2D COFs could be synthesized without deviation from the above described procedure, but additional acetic acid catalyst was required for the successful synthesis of 3D imine COFs. 3D-COF-HNU10 could not be obtained using traditional, solvothermal methods. 
Moreover, surface areas for all COFs were comparable or higher than values previously reported via solvothermal routes. Finally, two different DES, namely choline chloride/urea (ChCl/U), 1/2, and choline chloride ethylene glycol (ChCl/EG), 1/2, were tested. TFPM-PPD COF, which had a previously reported BET surface area of $535 \mathrm{~m}^{2} \mathrm{~g}^{-1}$ via solvothermal routes, was synthesized in all three DES. BET surface areas of $747 \mathrm{~m}^{2} \mathrm{~g}^{-1}, 805 \mathrm{~m}^{2} \mathrm{~g}^{-1}$ and $977 \mathrm{~m}^{2} \mathrm{~g}^{-1}$ were obtained for $\mathrm{ChCl} / \mathrm{Gly}, \mathrm{ChCl} / \mathrm{Eg}$ and $\mathrm{ChCl} / \mathrm{U}$, respectively.

3.3.2 Advanced acidic catalysts. While acetic acid has routinely been used in the synthesis of imine COFs, it not a very efficient catalyst for this reaction. However, Lewis acidic metal triflates proved very promising, providing highly crystalline and porous TAPB-BDA COF at room temperature in as little as 10 minutes (Fig. 19). ${ }^{177}$ High polymer yields could be obtained from various metal triflates $\left(\mathrm{Sc}(\mathrm{OTf})_{3}, \mathrm{Eu}(\mathrm{OTf})_{3}\right.$, $\mathrm{In}(\mathrm{OTf})_{3}, \mathrm{Yb}(\mathrm{OTf})_{3}, \mathrm{Y}(\mathrm{OTf})_{3}$ and $\left.\mathrm{Zn}(\mathrm{OTf})_{2}\right)$ in 5-150 min using $0.0001 \mathrm{eq}$. at room temperature. In contrast, no precipitation could be achieved after 14 days in equimolar amounts of acetic acid at $90{ }^{\circ} \mathrm{C}$. Subsequent $\mathrm{ScCO}_{2}$ activation and PXRD analysis revealed a highly intense (100) peak and the appearance of higher order reflections for $\mathrm{Sc}(\mathrm{OTf})_{3} . \mathrm{Eu}(\mathrm{OTf})_{3}, \operatorname{In}(\mathrm{OTf})_{3}$ and $\mathrm{Yb}(\mathrm{OTf})_{3}$ also performed reasonably well, with an intense (100) diffraction peak, while $\mathrm{Y}(\mathrm{OTf})_{3}$ and $\mathrm{Zn}(\mathrm{OTf})_{2}$ provided mainly to totally amorphous materials. Most likely, this is a consequence of the smaller ionic radii of Sc ions, as has been reported

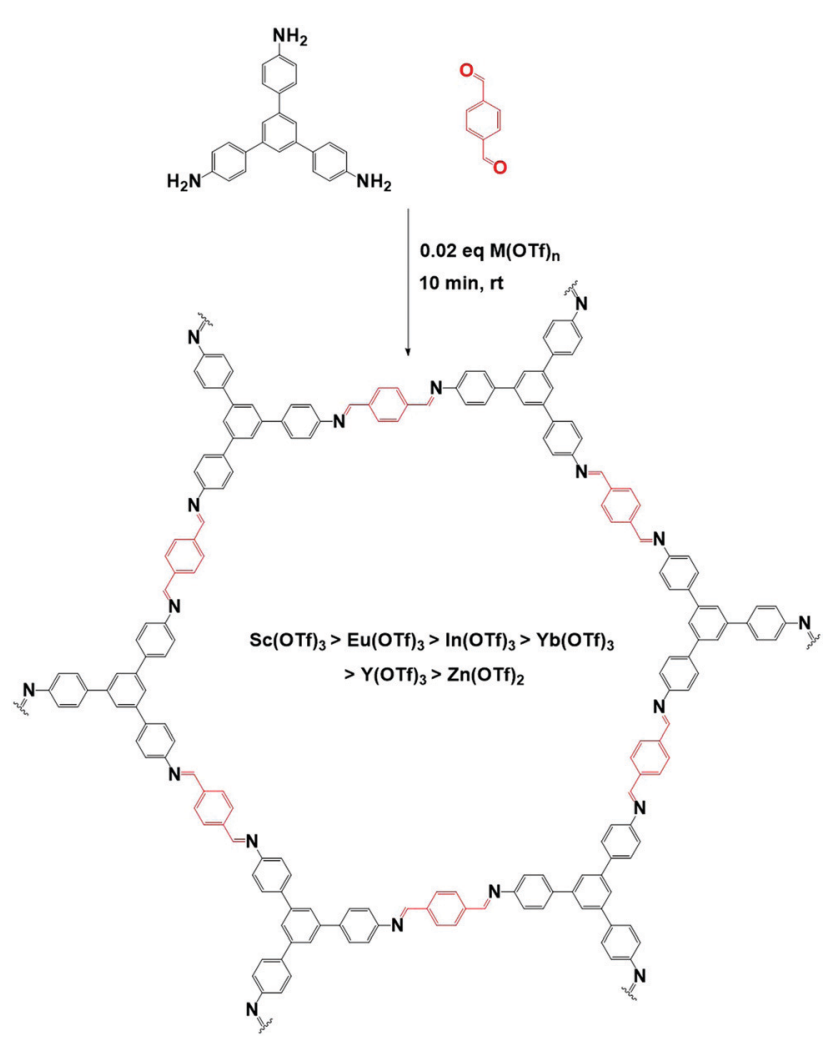

Fig. 19 Schematic representation of COF synthesis via metal triflate catalysis. TAPB-BDA COF is prepared in 10 minutes at room temperatures using 0.02 eq. of metal triflate. $\mathrm{Sc}(\mathrm{OTf})_{3}$ performed best, followed by $\mathrm{Eu}(\mathrm{OTf})_{3}, \ln (\mathrm{OTf})_{3}, \mathrm{Yb}(\mathrm{OTf})_{3}, \mathrm{Y}(\mathrm{OTf})_{3}$ and finally $\mathrm{Zn}(\mathrm{OTf})_{2}$. earlier. ${ }^{178}$ Next, loading of the catalyst was studied, leading to an optimized amount of 0.02 eq. Sharp and high intense diffraction peaks were obtained with a BET surface area of $2175 \mathrm{~m}^{2} \mathrm{~g}^{-1}$ (while classic acetic acid catalyzed synthetic protocol generally lead to surface areas below $1000 \mathrm{~m}^{2} \mathrm{~g}^{-1}$ ). Finally, the method was extended to two new COFs. ${ }^{177}$ Since then, this method has already been extended to multiple other imine COFs, ${ }^{154,179,180}$ with one report even achieving a one-pot cascade reaction to afford two quinoline linked COFs with high crystallinity and porosity. ${ }^{181}$

Alternatively, $p$-toluenesulfonic acid (PTSA) and derivatives could be used as both catalyst and molecular organizer. In a first study, PTSA was used to obtain 12 different COFs with high surface areas and crystallinity in very short timeframes. Moreover, the synthesis could be performed on a $10 \mathrm{~g} \mathrm{~h}^{-1}$ scale using a twin screw extruder. ${ }^{182}$ For this, the used diamine was first reacted with PTSA to form an amine salt. Subsequently, TFPM was added to the mixture and grinded for 10 minutes. Finally, $100 \mu \mathrm{L} \mathrm{H}_{2} \mathrm{O}$ was added and the mixed mixture was heated at $170{ }^{\circ} \mathrm{C}$ for one minute to obtain the COF. The whole procedure is shown schematically in Fig. 20. The material was isolated in high yield after dipping in hot water for five minutes. PXRD analysis revealed high intense (100) reflections and BET surface areas of up to $3000 \mathrm{~m}^{2} \mathrm{~g}^{-1}$ could be obtained, sometimes two to three times higher than previously reported values for these COFs. ${ }^{182}$ Mechanistic studies revealed the importance of $\mathrm{H}$-bonding in this process. When PTSA and the amine react, a H-bonded lamellar structure is formed, acting as a template for 2D COF formation. Later on, when TFPM is added, it can easily exchange PTSA due to the similar size of both compounds, in order to form crystalline COFs. Indeed, when other acids (phosphoric acid, trifluoromethyl carboxylic acid, 2-aminobenzenesulfonic acid, phenol sulfonic acid, 4-nitrobenzenesulfonic acid or benzenesulfonic acid) were used, much lower surface areas and crystallinity were observed, indicating the crucial role of molecular orientation and geometry in the formed amine salt. The influence of the amine salt structure on COF properties has been studied in more detail in a follow-up report, where the combination of TFPM with 11 different amines in presence of five PTSA derivates was followed. ${ }^{183}$ It was observed that in the formed amine salts, every amine functionality $\left(\mathrm{NH}_{3}{ }^{+}\right)$was surrounded by three $\mathrm{H}$-bounded $\left(\mathrm{N}_{\text {amine }}-\mathrm{H}-\mathrm{O}_{\text {acid }}\right)$ acid molecules, forming a $1 \mathrm{D}$ lamellar structure. After aldehyde addition, these H-bonds can compete with imine formation, indicating the importance of controlling $\mathrm{H}$-bond strength, as too weak H-bonds might not compete enough, while too strong H-bonds can impede COF formation. Indeed, a clear interaction between surface area and $\mathrm{H}$-bond distance was observed, with an optimal distance between 2.06 and $2.19 \AA$ resulting in COFs with the highest percentage of theoretical surface area.

Finally, trifluoromethanesulfonic acid could be used to synthesize CTFs at room temperature or via microwave radiation. Most notable advantages of this technique are the absence of metal/salt contamination, and the possibility to obtain colored materials incorporating functional groups that could not 
<smiles>O=Cc1c(O)c(C=O)c(O)c(C=O)c1O</smiles>

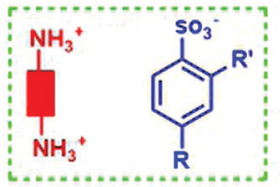

Amine salt

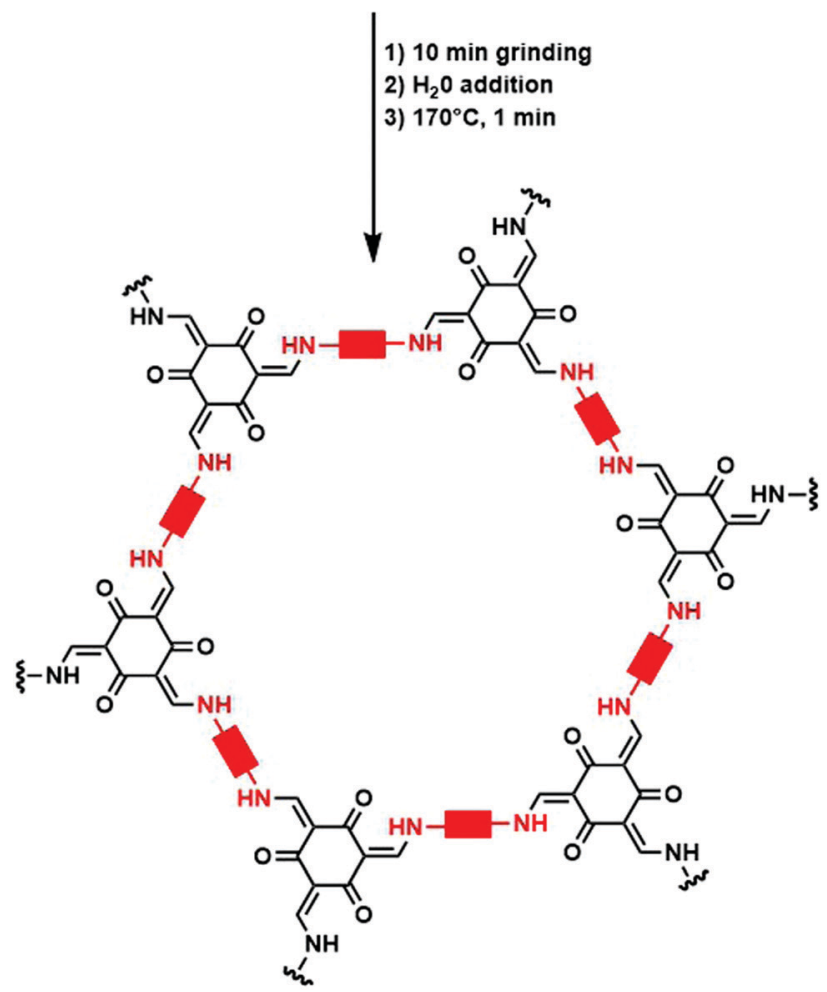

Fig. 20 Schematic representation of COF synthesis using $p$-toluenesulfonic acid and derivates as catalysts. First, the amine linker is reacted with the catalyst to generate an amine salt, which is then mixed via $10 \mathrm{~min}$ grinding with the aldehyde (TFPM). After $\mathrm{H}_{2} \mathrm{O}$ addition, the material is heated at $170{ }^{\circ} \mathrm{C}$ for one minute to obtain a crystalline COF.

withstand the harsh conditions typically used. However, pore sizes are moderate to low and only a few microwave-synthesized materials showed some crystallinity. Still, this indicates that an acid catalyst and high pressure could reach some reversibility at quite low temperatures. ${ }^{184}$

3.3.3 Other catalytic improvements. The first report of imide COFs (Pi-COF-1, Pi-COF-2 and Pi-COF-3) used high boiling organic solvents (like $N$-methyl-2-pyrrolidone) with isoquinoline as a catalyst for five to seven days. ${ }^{42}$ Recently, an easier method was developed using $\mathrm{ZnCl}_{2}$, as already routinely done in CTF synthesis, ${ }^{141}$ taking only 48 hours to crystallize the COF. ${ }^{185}$ To form Pi-COF-3 or TAPB-PTCDA COF, both linkers were combined with anhydrous $\mathrm{ZnCl}_{2}$ and reacted under vacuum for $48 \mathrm{~h}$ at $300{ }^{\circ} \mathrm{C}$, as shown in Fig. 21. It is worth noting that $\mathrm{ZnCl}_{2}$ melts just above $300{ }^{\circ} \mathrm{C}$, indicating the presence of highly mobile ions in the reaction mixture even though no melt is obtained yet at this temperature. In molten conditions, above $300{ }^{\circ} \mathrm{C}$, no crystalline material could be formed, as well as below $280{ }^{\circ} \mathrm{C}$. Moreover, the obtained crystallinity (at $300{ }^{\circ} \mathrm{C}$ ) increased with concentration of $\mathrm{ZnCl}_{2}$ until 12.5 eq., suggesting that 12.5 eq. was

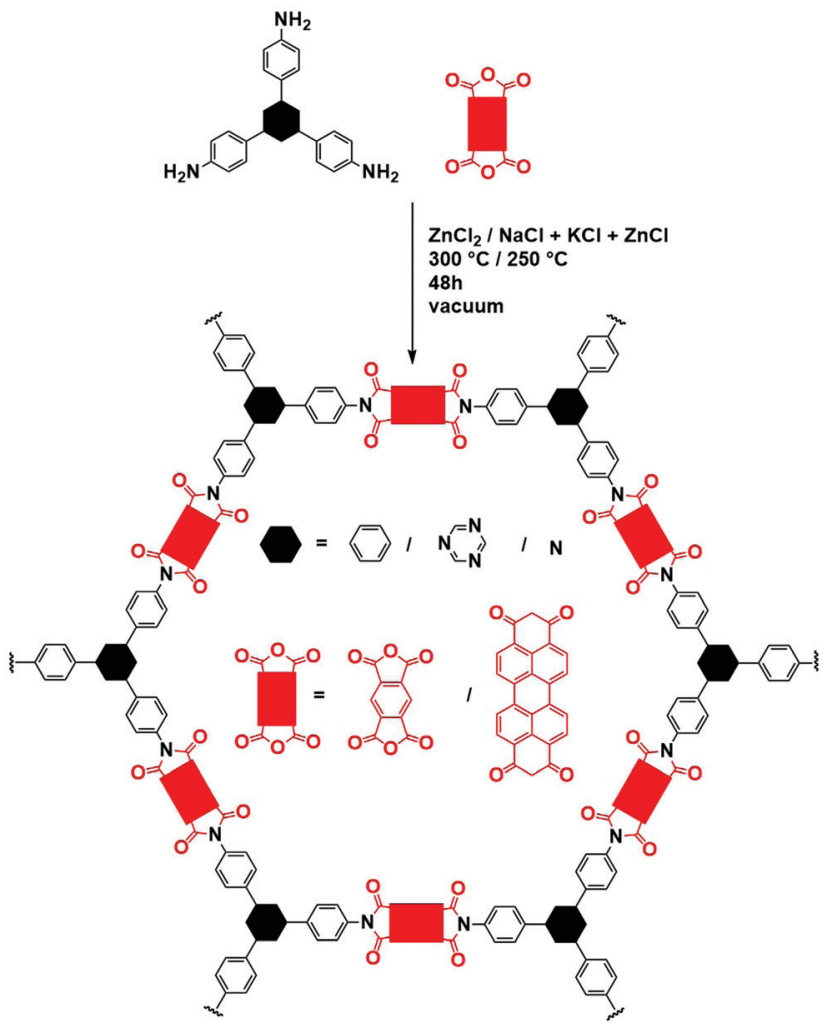

Fig. 21 Schematic representation of imide COF synthesis in $\mathrm{ZnCl}_{2}$. Black hexagons represent the different moieties used as amine, red rectangles the different dianhydrides.

enough to reach full accessibility. Finally, while 48 hours of reaction time was used, crystalline compounds could already be observed after five hours and no evolution in crystallinity was seen anymore after 10 hours. Still, the temperature of $300{ }^{\circ} \mathrm{C}$ could destroy fragile linkers, like triazine bearing compounds. Therefore, an alternative procedure was developed using an eutectic mixture of $\mathrm{ZnCl}_{2}, \mathrm{NaCl}$ and $\mathrm{KCl}$. As this mixture has a lower melting point, reaction temperature could be lowered to $250{ }^{\circ} \mathrm{C}$, making the synthesis of imide COFs with more fragile linkers possible. However, no clear improvement in quality compared to the "classical" method could be obtained. For some COFs the obtained surface areas were higher, while for others they were comparable or even very low (which might be assigned to pore blocking by residual $\mathrm{ZnCl}_{2}$ ).

Base catalyzed imine COF formation was proven possible for four different materials. ${ }^{186}$ However, it has to be noted that a phenol function on the aldehyde linker is necessary for this protocol. An aqueous solution of aldehyde and $\mathrm{KOH}$ was prepared to which the amine was added in organic solvent ( $N, N$-diethylformamide, DEF or $N, N$-dimethylformamide, DMF). Sharp PXRD reflections and moderate porosities were obtained, however, materials prepared using PTSA catalysis (as explained in Section 3.3.2) were of better quality. It was also observed that a large interval for crystallinity existed, as the concentration of $\mathrm{KOH}$ could be altered between 2 and 6 eq. and the $\mathrm{DEF} / \mathrm{H}_{2} \mathrm{O}$ ratio between $1 / 1$ and $3 / 1$ to yield highly crystalline compounds. 
Moreover, $\mathrm{KOH}$ and $\mathrm{CsOH}$ could also be used, with the main difference being the salt formed by combination of base and phenol containing aldehyde. Highly soluble salts were preferred, which were then slowly reacted to imines in a large excess of water.

Another method used compressed $\mathrm{CO}_{2}$ in $\mathrm{H}_{2} \mathrm{O}$ as solvent for COF formation, which is obviously an attractive option due to environmental reasons. ${ }^{187}$ Most likely, in situ formation of acidic $\mathrm{H}_{2} \mathrm{CO}_{3}$ is important to catalyze the COF formation, but the low viscosity of this solvent might also be important, as pure aqueous acetic acid solutions did not yield crystalline materials. LZU-1 was prepared in $24 \mathrm{~h}$ at room temperature and could easily be obtained by $\mathrm{CO}_{2}$ depressurization. An increase of crystallinity with $\mathrm{CO}_{2}$ pressure was observed, with sharper and more intense (100) reflections, 4.5 MPa was used as an optimized value. While BET surface areas for lower (2.4 and $3.5 \mathrm{MPa}) \mathrm{CO}_{2}$ pressures were very low $\left(23\right.$ and $42 \mathrm{~m}^{2} \mathrm{~g}^{-1}$ respectively), a value of $678 \mathrm{~m}^{2} \mathrm{~g}^{-1}$ was obtained at $4.5 \mathrm{MPa}$, which is significantly higher than the $410 \mathrm{~m}^{2} \mathrm{~g}^{-1}$ for the 'classical' solvothermally synthesized material.

\subsection{Interfacial COF growth and solubility dependency}

While solvent selection and ratios have decisive roles in the crystallization of COFs, not many systematic studies have been performed on the effect of solvents on COF quality and the underlying mechanisms. Most often trial-and-error approaches are used to select appropriate solvents, loosely based on conditions used in the first reports. For example, mesitylene/ dioxane mixtures are commonly used in order to control linker solubility and slow down the reaction. ${ }^{1,23}$ One theoretical study reported a difference in binding energy in different solvents, ${ }^{107}$ which theoretically can lead to the growth of large single crystals. ${ }^{188}$ But no direct origin for the obtained values could be found. ${ }^{107}$ A more recent example is the "ventilation vial" approach to COF-300, using a cyclohexane and 1,4-dioxane mixture as reaction solvent. ${ }^{189}$ During reaction, dioxane is vented out of the mixture, generating a gradient of acidity and polarity over time. This reduces solubility and increases acidity, leading to highly crystalline COF. The method could easily be scaled up to two gram scale. Finally, it has been shown that both boroxine e.g. COF-5) $)^{109}$ and imine COFs e.g. TAPB$\mathrm{BDA} \mathrm{COF})^{170}$ can be stabilized as colloidal suspensions by using Lewis basic solvents (e.g. ACN), as already discussed in Section 2.1.

However, most of the studies regarding solubility have been focused on thin film growth. Multiple reports have shown that highly crystalline thin films can readily be obtained by interfacial COF growth. In a first report, a solution of linkers in $\mathrm{CHCl}_{3}$ was carefully put on a water layer, synthesizing the COF at the air/water interface. ${ }^{190}$ Overnight a smooth polymer layer was formed, but no crystallinity or porosity was reported, indicating insufficient reversibility or too fast COF formation. In later reports, this issue was solved by the separation of reagents and/or catalyst in different phases with $\mathrm{COF}$ formation on the interface. These procedures are schematically shown in Fig. 22.

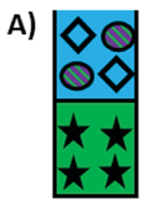

B)

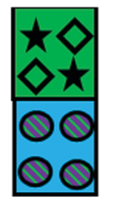

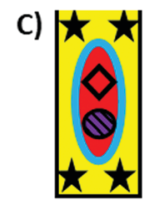

D)

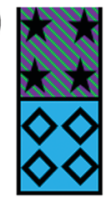

$=\mathrm{H}_{2} \mathrm{O}$

= Organic phase

$=$ Oil

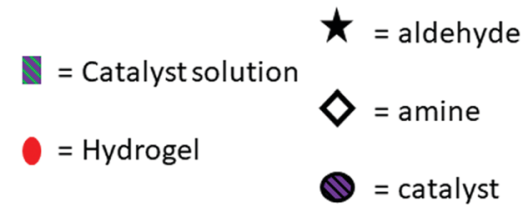

Fig. 22 Schematic representation of different ways to prepare crystalline imine COF thin films. (A) Combining amine and catalyst in the aquatic phase with aldehyde in an organic phase. (B) Combination of both amine an aldehyde linkers dissolved in organic solvent with catalyst in $\mathrm{H}_{2} \mathrm{O}$. (C) Inclusion of amine linkers and catalysts in a hydrogel dipped in an aldehyde containing oil, COF formation happens in a water layer at the oil/hydrogel boundary. (D) Amine linkers dissolved in water are combined with aldehydes dissolved in organic catalyst.

A first approach (Fig. 22A) was based on the earlier mentioned PTSA catalyzed COF synthesis (Section 3.3.2). Aldehyde linkers were dissolved in dichloromethane (DCM), followed by addition of $\mathrm{H}_{2} \mathrm{O}$ as spacer and finally a $\mathrm{H}_{2} \mathrm{O}$ solution of amine salt was put on top. ${ }^{191} \mathrm{~A}$ thin film of Tp-Tta or Tp-Ttba is formed at the interface over 72 hours at room temperature. Crystalline films could be obtained due to the intermediate layer of pure $\mathrm{H}_{2} \mathrm{O}$, which slows down the diffusion of linkers to the interface even more. This way, sharp PXRD reflections and surface areas which are among the highest values noted for 2D thin films are obtained. Four different COFs were tested for this method and film thickness could easily be varied (from $50 \mathrm{~nm}$ to a few $\mu \mathrm{m}$ by changing monomer concentration. Finally, SEM analysis showed that fibers are formed rapidly $(1 \mathrm{~h})$, which slowly assemble to crystalline sheets (from $24 \mathrm{~h}$ onwards). The other catalyst mentioned in Section 3.3.2, Sc(OTf $)_{3}$, could also be used for thin film synthesis (Fig. 22B). ${ }^{179}$ Here, both precursors (aldehyde and amine) could be dissolved in organic solvent, while the catalyst resides in the water layer. After $72 \mathrm{~h}$, thin films of TAPB-BDA COF could easily be separated from the mixture using tweezers. Again, film thickness could be controlled by varying monomer concentration, opening a range from $20 \mathrm{~nm}$ to $100 \mu \mathrm{m}$. However, it has to be noted that reaction times increased for thinner films. Remarkably, even thinner films (up to $2.5 \mathrm{~nm}$ ) could be formed rapidly by the use of very small amounts of organic solvent, but no crystallinity could be obtained for those films. The same procedure was also used to prepare thin films by Suzuki polymerization. ${ }^{192}$ Again, both linkers where in the organic phase, while the catalyst $\left(\mathrm{K}_{2} \mathrm{CO}_{3}\right)$ was dissolved in $\mathrm{H}_{2} \mathrm{O}$. Suzuki reactions are very attractive due to the wide scope of available monomers, mild conditions and resulting strong C-C bonds. However, the irreversibility of this reaction makes it fundamentally difficult to apply in COF synthesis. Indeed, no crystallinity could be observed in XRD analysis of the resulting materials. Still, HR-TEM analysis 
showed periodicity and a layered structure, indicating some order in small domains. Fig. 22C illustrates a method to grow COF thin films with the use of hydrogels. ${ }^{193}$ First, a solution of amine in aqueous acetic acid was allowed to be incorporated in a hydrogel. Subsequently, the swollen hydrogel was put in an oil solution of aldehyde linker and a small amount $(10 \mu \mathrm{L})$ of $\mathrm{H}_{2} \mathrm{O}$ was added. Thin films with thickness of 4 to $150 \mathrm{~nm}$ (by monomer concentration variation) formed over $12 \mathrm{~h}$ at the oil/water/hydrogel interface. Observed XRD reflections were in good agreement with previously obtained PXRD patterns for COF TTA-DHTA and allowed to calculate a grain size of $27.5 \mathrm{~nm} \pm 0.4 \mathrm{~nm}$. Thin film synthesis was also attempted at a liquid/liquid interface, but obtained products were amorphous. This was attributed to delayed diffusion by the hydrogel as amine diffusion only reached equilibrium after $1 \mathrm{~h}$. Very recently, COF thin films have been synthesized at the interface of an amine in $\mathrm{H}_{2} \mathrm{O}$ solution with an aldehyde/catalyst mixture (octanoic acid was used as a catalyst), as illustrated in Fig. 22D. ${ }^{194}$ No organic solvents were needed as the aldehyde could directly be dissolved in the catalyst. This led to rapid formation of an amorphous thin film at the interface, which subsequently acted as a diffusion barrier. The resulting decreased linker concentration in combination with the strong affinity of NUS-9 to the used solvents resulted in slow exchange to highly crystalline membranes.

\subsection{Exchange reactions}

Exchange reactions in COF formation can be separated into two distinct categories with different goals. In amorphous to crystalline exchange, the exchange is the deciding factor to reach crystallinity. This is highly advantageous for creating COFs with unique morphologies or composite materials. First, an amorphous polymer is rapidly formed in the desired morphology, which is then allowed to slowly exchange to a crystalline COF overtime. Crystalline to crystalline exchange however can be used to incorporate linkages which are not directly accessible for COF synthesis or yield materials with low crystallinity. Initially, a highly crystalline COF is formed of which some linkers are later exchanged to the desired (functional) linkers, often obtaining increased crystallinity.

3.5.1 Amorphous to crystalline exchange. Amorphous imine polymers could be formed around a $\mathrm{Fe}_{3} \mathrm{O}_{4}$ nanocluster by $2 \mathrm{~h}$ reflux of the monomers in THF. ${ }^{117}$ Subsequently, the amorphous polymer was rearranged to crystalline TFPM-BZ COF by three days reaction in $o$-dichlorobenzene (DCB)/butanol $(9 / 1)$ at $120{ }^{\circ} \mathrm{C}$. As aqueous acetic acid partially destroyed the $\mathrm{Fe}_{3} \mathrm{O}_{4}$ nanocluster, pyrrolidine was used as a catalyst. PXRD measurements showed the appearance of COF reflections in addition to the pre-existing $\mathrm{Fe}_{3} \mathrm{O}_{4}$ peaks, while BET surface area increased from $255 \mathrm{~m}^{2} \mathrm{~g}^{-1}$ (for the amorphous polyimine on $\mathrm{Fe}_{3} \mathrm{O}_{4}$ ) to $1346 \mathrm{~m}^{2} \mathrm{~g}^{-1}$ (pure $\mathrm{Fe}_{3} \mathrm{O}_{4}$ has a surface area of $123 \mathrm{~m}^{2} \mathrm{~g}^{-1}$ ). Remarkably, pyrrolidine catalyzed bulk COFs also showed improved surface area over the aqueous acetic analogue, with values of $1883 \mathrm{~m}^{2} \mathrm{~g}^{-1}$ and $620 \mathrm{~m}^{2} \mathrm{~g}^{-1}$, respectively. Thickness of the formed COF shell could easily be controlled and varied (from 20 to $100 \mathrm{~nm}$ ) by varying monomer concentration in the initial polyimine condensation. Another report studied the encapsulation on nanoparticles in COFs. ${ }^{195}$ Here, pre-made nanoparticles (NP) were added to an aldehyde/acetic acid solution of TABP-BTCA COF monomers and allowed to react at room temperature (RT) for $1 \mathrm{~h}$, incorporating the nanoparticles in COF spheres. Afterwards, the samples were put in dioxane/mesitylene, aqueous acetic acid was added and the mixture was refluxed for seven days, resulting in crystalline materials. This way, $\mathrm{Fe}_{3} \mathrm{O}_{4}, \mathrm{Au}$ and $\mathrm{Pd}$ nanoparticles could be incorporated and the resulting materials were shown to be crystalline and porous. It was also shown that the amorphous to crystalline exchange could be used in the preparation of fibrous COFs. ${ }^{196}$ To achieve these materials, amines were added dropwise in an ethanol solution of aldehyde linkers. After $24 \mathrm{~h}$ reflux, the formed amorphous fibers were filtered off and transferred to a DCB/butanol mixture in which acetic acid catalyzed rearrangement occurred over three days at $120{ }^{\circ} \mathrm{C}$. The obtained COF fibers showed crystallinity and porosity, but the quality is reduced in comparison to "common" spherical COFs (less intense and broader PXRD reflextions, BET surface area of 295 instead of $782 \mathrm{~m}^{2} \mathrm{~g}^{-1}$ ).

Zhang et al. found out that, while COFs cannot be used directly for 3D printing, this was possible for the amorphous polymers initially formed. ${ }^{197}$ Linkers, PTSA and Pluronic ${ }^{\circledR} 127$ (F127) were mixed in $\mathrm{H}_{2} \mathrm{O}$ /THF solution, out of which the THF rapidly evaporated. The resulting hydrogel was used for $3 \mathrm{D}$ printing of a pyramid, which was heated to $90{ }^{\circ} \mathrm{C}$ for $24 \mathrm{~h}$ to remove F127 and later on crystallized in dioxane/mesitylene at 150 (72 h, acetic acid catalyst, annealing). TpPA-1 and TpPA-2 (or 3D-TpBD- $\mathrm{Me}_{2}$ ) were synthesized with comparable crystallinity and surface area as the pristine materials, the process and resulting PXRD pattern can be found in Fig. 23a and c, respectively. 3D-TPE-COF was also tested, but needed modulator addition to slow down the synthesis enough to allow 3D printing (Fig. 23b and d). Finally, it was shown that different COFs could be combined and bound at the interface, suggesting connections at the molecular level, while even retaining some crystallinity (Fig. 23e-g).

Crystalline COFs could also be prepared by linkage type substitution from amorphous porous organic polymers (POPs). ${ }^{198}$ In a first step, imine and imide linked POPs were prepared, to which dialdehydes and dianhydrides were added respectively. When these mixtures were put in typical COF growth conditions for the desired linkage type, crystalline COFs were obtained (Fig. 24). Crystallinities and porosities for all obtained materials were high, Exch-COF-1 and Exch-COF-4 even showed much higher BET surface areas than could be obtained by direct synthesis (1089 vs. $796 \mathrm{~m}^{2} \mathrm{~g}^{-1}$ for Exch-COF-1 and 2238 vs. 1700 for Exch-COF-4). However, it has to be noted that linker exchange with terephtaldehyde from the polyimine was not successful, possibly due to the high stability of the imide bond. The used $\mathrm{OH}$-functionalized aldehydes could overcome this issue by non-covalent stabilization of the imine bond. The formation of COF-1 could be tracked over time with FTIR, SEM, PXRD and $\mathrm{N}_{2}$-sorption, showing that while transformation started fast (appearance of a weak diffraction peak after $2 \mathrm{~h}$ ), it was only complete after five days. 

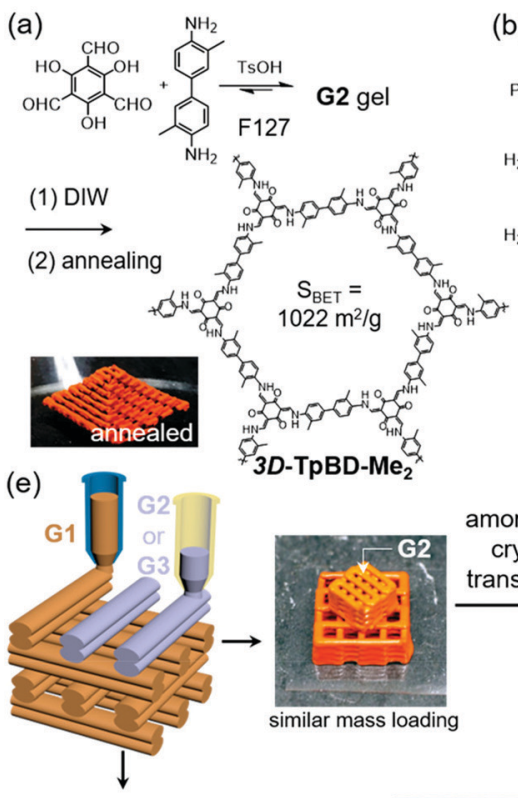

$3 D-\mathrm{TpBD}-\mathrm{Me}_{2}$

(b)

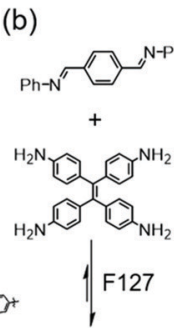

G3 gel
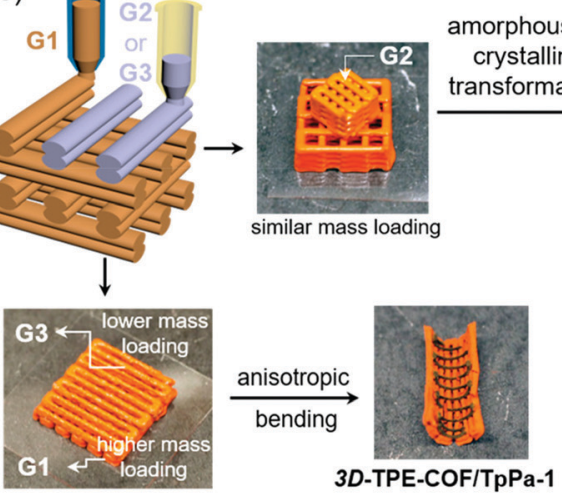

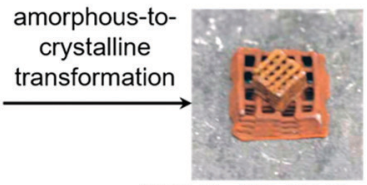

3D-TpPa-1/TpBD-Me ${ }_{2}$

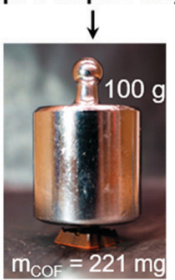

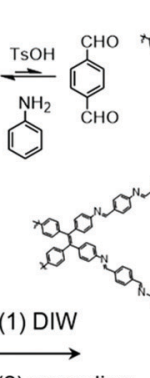

(2) annealing
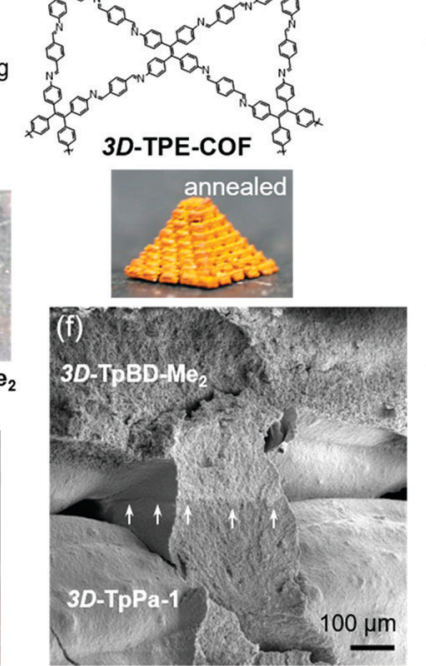

(c)

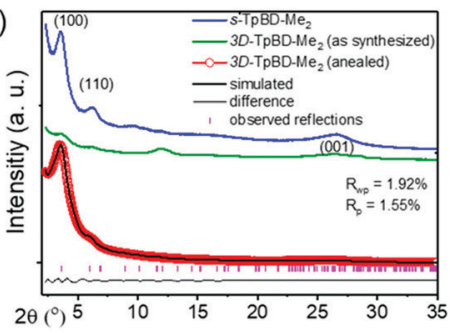

(d)

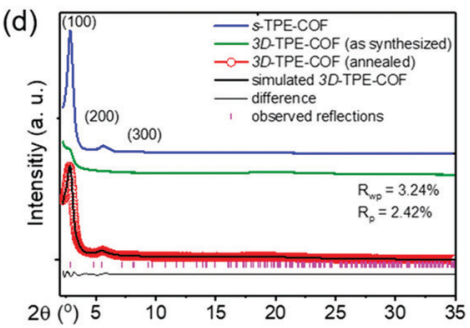

(g)

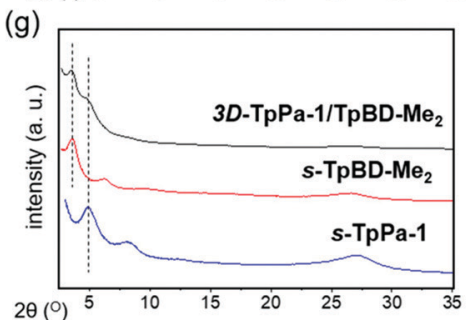

Fig. 23 (a and b) Schematic representation of the used procedure for 3D printed $\beta$-ketoenamine COF (3D-TPBD-Me 2 ) and imine COF (3D-TPE-COF). ( $c$ and d) PXRD patterns of as-synthesized and annealed $\beta$-ketoenamine and imine COF. (e) Heterogeneous printing of two different COF and their obtained lattices (3D-TpPa-1/TpBD-Me 2 ) and (3D-TPE-COF/TpPa-1), the materials could withstand anisotropic bending and a weight of $100 \mathrm{~g}$. (f) SEM image of the interface of heterogeneous 3D-TpPa-1/TpBD-Me 2 . $(\mathrm{g})$ PXRD patterns of pristine TpBD-Me $\mathrm{M}_{2}$ and TpPa-1 compared to the pattern of heterogeneously printed 3D-TpPa-1/TpBD-Me 2 . Reproduced with permission from ref. 197, Copyright American Chemical Society (2019).

3.5.2 Crystalline-to-crystalline exchange. In a first example of crystalline-to-crystalline exchange, two different triple-pore COFs were synthesized from condensation of $\left[1,1^{\prime}: 3^{\prime}, 1^{\prime \prime}\right.$ -

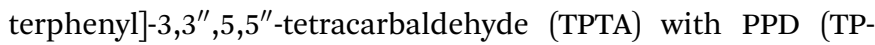
COF-DAB) or benzidine (TP-COF-BZ). ${ }^{73}$ Next, as PPD should be more active than benzidine due to the increased amount of electron-donating amine groups, linker exchange was performed, as shown in Fig. 25. Suspensions of TP-COF-BZ in COF growth conditions were prepared and 1,4-diaminobenzene was added. Using PXRD analysis, it was observed that linker exchange was complete when 10 eq. of 1,4-diaminobenzene was used, obtaining almost identical PXRD patterns as directly synthesized TP-COF-DAB. Here, it is important to note that, compared to TP-COF-DAB, TP-COF-BZ showed reduced crystallinity, indicating an improvement of crystallinity in the exchange process. Time-dependent PXRD experiments confirmed that the COF-to-COF transformation happens rapidly, as TP-COF-BZ peaks already disappeared after $0.5 \mathrm{~h}$ and first TP-COF-DAB peaks could be observed after $1 \mathrm{~h}$. Furthermore, after $4 \mathrm{~h}$, no significant differences could be observed anymore. These results were confirmed by ${ }^{1} \mathrm{H}$ NMR measurements on the COF, where $54.55,88.89$ and $95.49 \%$ of 1,4-diaminobenzene was found after $0.5,1$ and $4 \mathrm{~h}$, respectively. Moreover, no COF dissolution was observed, indicating the heterogeneous nature of this process, accelerated by the porous nature of the COF and acetic acid catalysis. A similar process could be used on the COFs formed by 1,3,5-tris(4-formylphenyl)triazine (TFPT) and PPD (PTPA-COF) or benzidine (PTBD-COF). ${ }^{199}$ Here, the benzidine was exchanged for 1,2,4-benzenetriamine and the PPD for 3,3'-diaminobenzidine, generating a COF decorated with free amine functions. This is almost unreachable in direct imine COF synthesis, as amines condensate with aldehydes to form the imine linkages. However, in a COF-to-COF transformation the framework is unaltered, making the inclusion of free amine groups possible. Optimal conditions were found to be $40{ }^{\circ} \mathrm{C}$ for three days in a THF/dimethylacetamide/aqueous acetic acid mixture. As 1,2,4-benzenetriamine is a better nucleophile than benzidine, 1 eq. was sufficient, while 10 eq. of 3,3'-diaminobenzidine were needed to generate a crystalline material. While crystallinity was retained in the COF-to-COF transformation, porosity of the obtained materials was reduced in comparison to the parent material.

In another study, two imine COFs based on the condensation of BTCA with PPD (COF-Ph) and 1,4-diaminonaphthalene (COF-Naph) were studied. ${ }^{200}$ Interestingly, while COF-Naph crystallized as a dense material, hollow particles were obtained for COF-Ph. Next, COF-to-COF transformation was performed, different equivalents of 1,4-diaminonaphthalene were added to COF-Ph. It was observed that both porosity and crystallinity improved as a result of the transformation, with sharper PXRD 

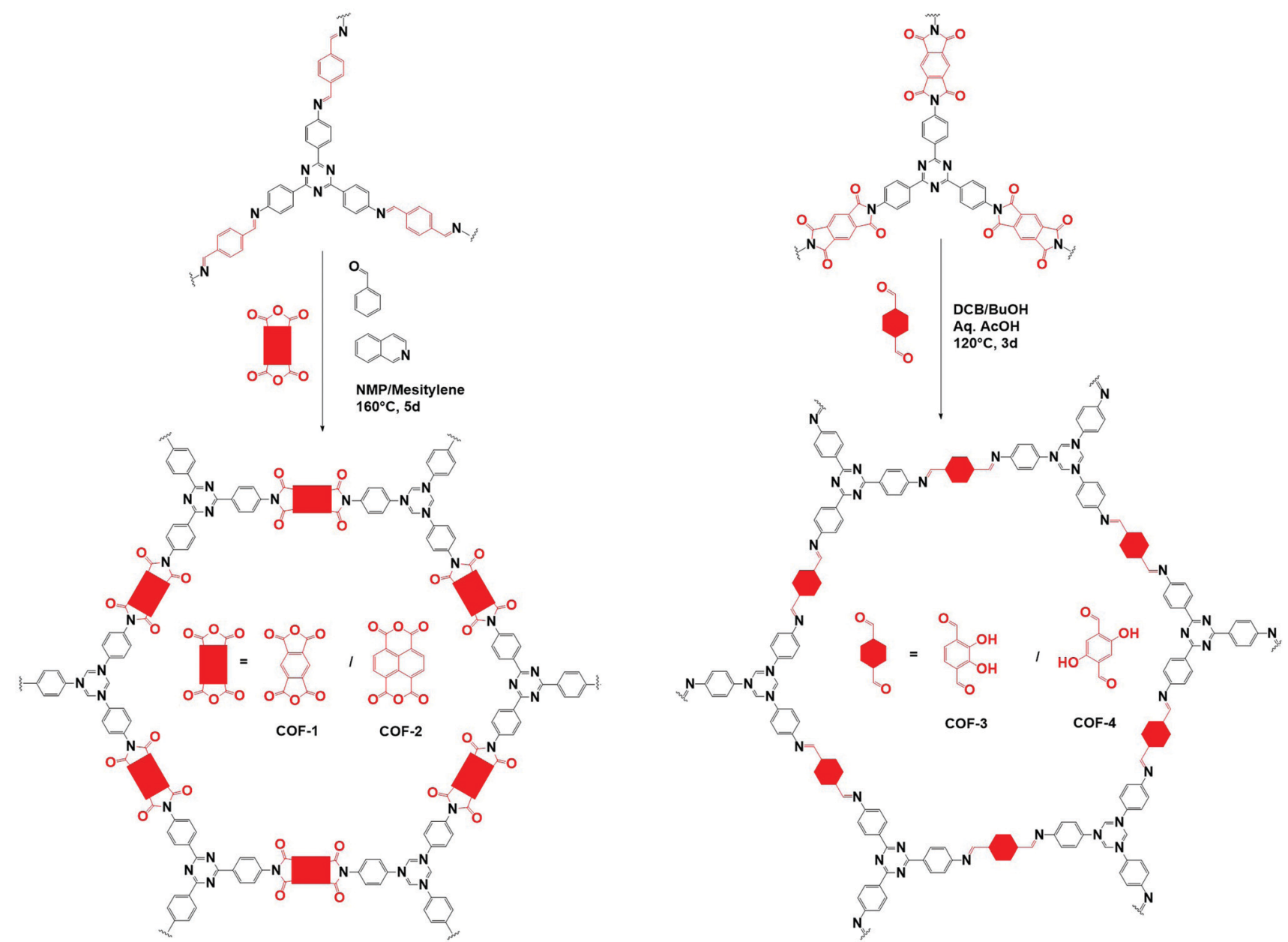

Fig. 24 Schematic illustration of the conversion of amorphous imine/imide polymer to four crystalline COFs. Imide COFs (COF-1 and COF-2) are formed by reaction of amorphous polyimine with dianhydrides in an NMP/mesitylene mixture $\left(160^{\circ} \mathrm{C}, 5\right.$ days, benzaldehyde and isoquinoline are added as catalysts). Imine COFs (COF-3 and COF-4) are prepared by acetic acid catalyzed exchange of dialdehydes with imide polymer in DCB/BuOH mixture (3 days, $120^{\circ} \mathrm{C}$ ).

reflections and higher BET surface areas for higher amounts of 1,4-diaminonaphthalene. NMR and TEM analysis showed that benzene and naphtalene rings were homogeneously mixed in the resulting material. In contrast, when PPD was added to COF-Naph, the dense starting material transformed to hollow spheres with small rod-shaped crystals embedded on. Additionally, NMR analysis revealed separate domains of COF-Ph and COF-Naph in the material. Still, both crystallinity and porosity improved in comparison to the parent material. Finally, it was observed that it was harder to exchange 1,4-diaminonaphthalene to PPD due to the strong $\pi$-stacking interactions in COF-Naph. This resulted in relatively less exchanged linker and even dissolution of the COF when too large an excess of PPD was used.

It was also proven possible to change linkage type by COF-toCOF transformation. This has been shown for the transformation of imine to $\beta$-ketoenamine linkages, as shown in Fig. $26 .{ }^{201}$ As imine formation is more reversible, this allows to obtain the highly stable $\beta$-ketoenamine linkage with improved crystallinity. Interestingly, a partly imine, partly $\beta$-ketoenamine linked material could be obtained by using 0.5 eq. of TFPM. However, the best porosity was obtained with exactly one equivalent, indicating that this is enough to perform the enamine-forming tautomerization step. The method resulted in sharper PXRD reflections and higher BET surface area $\left(1536 \mathrm{~m}^{2} \mathrm{~g}^{-1}\right.$ instead of $1102 \mathrm{~m}^{2} \mathrm{~g}^{-1}$ obtained by benzophenone monomer protection (as mentioned in Section 3.2.1) for BND-TFPM COF. Moreover, as a control experiment, $\beta$-ketoenamine COF was prepared from benzaldehyde protected amines, mimicking the transimination without the effect of pre-made frameworks. As both crystallinity and porosity for this control turned out to be decreased in comparison to the monomer exchange method, it was concluded that the pre-made COF framework was important to reach high-quality material. Comparable results were obtained for a recently published imine to amide exchange method, converting a reversible linkage to an irreversible variant for the first time. ${ }^{180}$ Here, the initial 4,4'-biphenyldicarboxaldehyde was exchanged with terephthaloyl chloride to form a highly stable amide COF. It has to be noted that it was impossible to build the obtained amide COF via direct condensation. For optimized results, the initial imine 


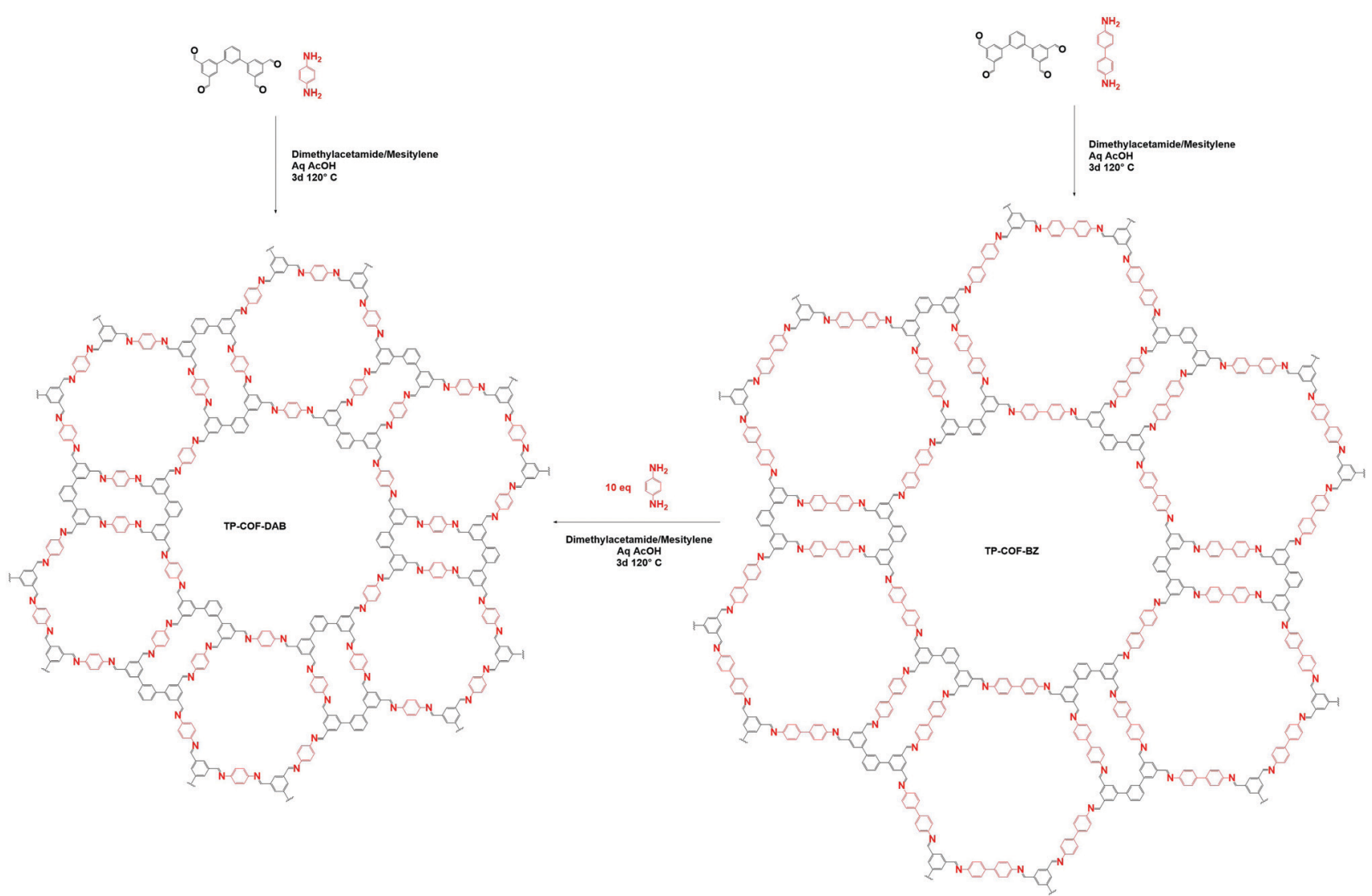

Fig. 25 Schematic illustration of the synthesis of two triple-pore COFs (TP-COF-DAB and TP-COF-BZ). It was shown that TP-COF-BZ could be transformed to TP-COF-DAB by linker exchange with 10 eq. of 1,4-diaminobenzene.
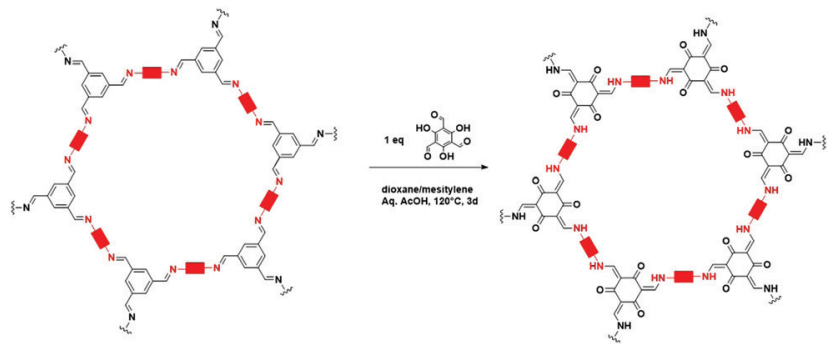

Fig. 26 Schematic representation of the synthesis of $\beta$-ketoenamine COF by linker exchange from imine COFs.

COFs were synthesized using $\mathrm{ScOTf}_{3}$ as a catalyst since the $\mathrm{H}_{2} \mathrm{O}$ in the commonly used aqueous acetic acid solution has a negative influence on the acid chloride used in the amide exchange reaction. Additionally, a longer aldehyde (more phenyl rings) was exchanged by a shorter acid chloride, to promote the reaction by insertion of a more nucleophilic building block. When 1 eq. of terephthaloyl chloride was used at low temperature $\left(0-4{ }^{\circ} \mathrm{C}\right)$ in presence of triethylamine, a highly crystalline amide COF was formed in two days. This was confirmed by highperformance liquid chromatography (HPLC) analysis of the reaction mixture before and after exchange, showing that 4,4'-biphenyldicarboxaldehyde replaced almost all terephthaloyl chloride after two days, while no concentration change was observed when no terephthaloyl chloride was added. Interestingly, more equivalents of terephthaloyl chloride or higher exchange temperatures resulted in dramatically reduced intensity of PXRD reflections. Finally, it has to be noted that both crystallinity (a tenfold increase in the main PXRD reflection) and porosity (156 to $261 \mathrm{~m}^{2} \mathrm{~g}^{-1}$; while direct synthesis of the amide COF gave a material with a surface area of $33 \mathrm{~m}^{2} \mathrm{~g}^{-1}$ ) increased from imine to amide COF.

Technically, the transformation of $1 \mathrm{D}$ ribbons to 2D COFs can also be seen as a COF-to-COF transformation via linker exchange. ${ }^{76}$ While no linkers are removed from the framework, new linkers are added, connecting the ribbons to form crystalline COFs. First, 1D ribbons were formed by the condensation of 1,3,6,8-tetrakis( $p$-formylphenyl)pyrene (TFPPy) and TFPA in the presence of $p$-toluidine as a modulator, rendering a network with unreacted amino groups (COF-76) (Fig. 27A). Here, cylindrical pores with 12 A diameters are already observed, as well as amine-imine interactions $\left(d_{\mathrm{H}-\mathrm{N}}\right)=3 \AA$, resulting in a BET surface area of $860 \mathrm{~m}^{2} \mathrm{~g}^{-1}$. Next, COF-76 was reacted with a dialdehyde or dianhydride to form an imine (COF-77) or imide (COF-78) linked COF, respectively (Fig. 27B). This transformed the $1 \mathrm{D}$ ribbons into $2 \mathrm{D}$ COFs with the appearance of a new type of pore (diameter $\approx 20 \AA$ ), resulting in increased BET surface areas $\left(1288 \mathrm{~m}^{2} \mathrm{~g}^{-1}\right.$ and $1080 \mathrm{~m}^{2} \mathrm{~g}^{-1}$, for COF-77 and COF-78, respectively). It has to be noted that COF-77 and COF-78 could 


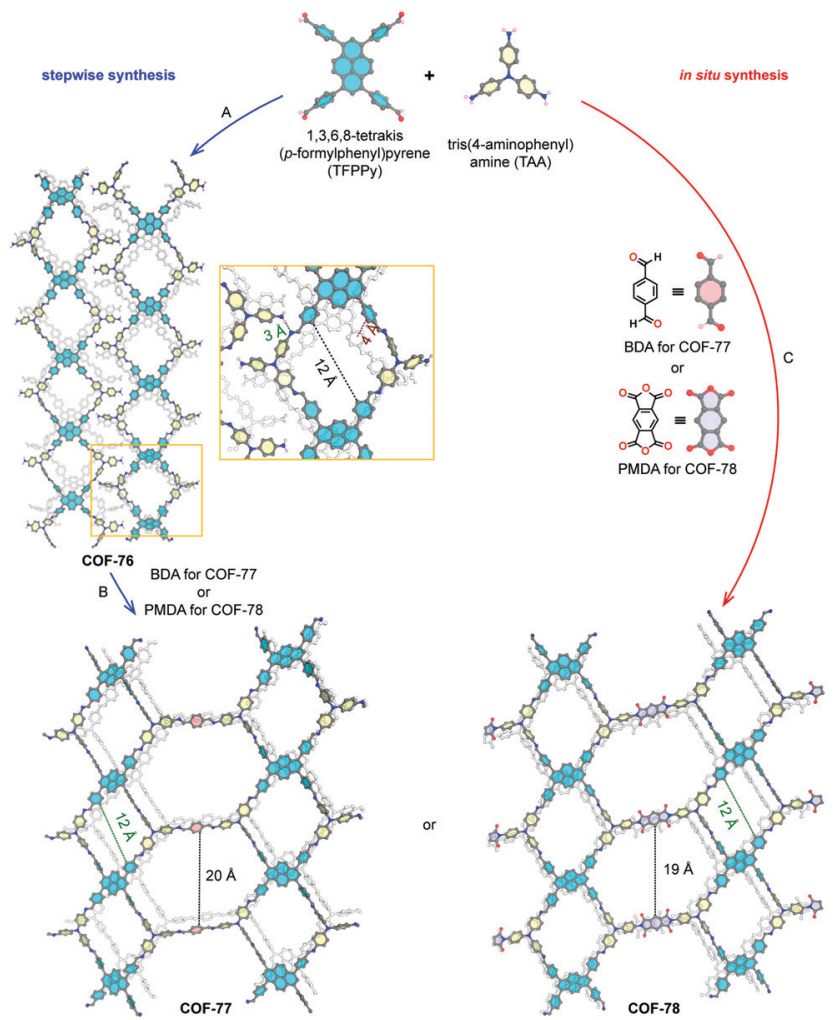

Fig. 27 Schematic route for the synthesis of COF-77 and COF-78. These materials can be obtained stepwise via $1 D$ ribbons ( $A$ and $B$ ) or in situ (C). Atom sphere colors: $\mathrm{C}$, gray; N, blue; O, red. Hydrogen atoms, except for aldehyde- and amine-hydrogens, are omitted for clarity. Reproduced with permission from ref. 76, Copyright American Chemical Society (2020).

also be formed by direct, in situ, condensation (Fig. 27C) with comparable crystallinity and porosity to the stepwise synthesis (BET surface area of COF-78 from in situ synthesis was even slightly higher, $1265 \mathrm{~m}^{2} \mathrm{~g}^{-1}$ ).

\subsection{Linker and condition optimization}

Much research has been done on optimizing linkers and conditions in order to find combinations resulting in the highest quality. However, most often, these methods cannot be generalized as they are highly dependent on the used linkers or based on easy to crystallize linkers. Still, the following section will give a short overview of interesting observations.

Firstly, planarity of the linker is a deciding factor in quality of the resulting COF. It has been observed that linkers incorporating the more planar triazine ring afford materials with higher crystallinity and porosity than phenyl ring based linkers. ${ }^{202-206}$ In Fig. 28B and C the reduced planarity of the phenyl core and its effect on the stacking energy is shown. This leads to less intense (100) PXRD reflections and slightly deformed crystallites (Fig. 28B and C). Moreover, the use of triazine rings makes it possible to induce donor acceptor stacking, which is more energetically favorable than $\pi-\pi$ stacking. ${ }^{204}$ It has to be noted that this observation is not limited to triazine/phenyl building blocks. In fact it has been shown that functionalization of a phenyl ring causes similar effects. ${ }^{207}$
Another important factor is the rigidity of the building blocks, as more flexible linkers lead to more possible conformations and thus lower crystallinity. ${ }^{208,209}$ However, both effects can be countered by the introduction of extra favorable interactions like hydrogen bonding. ${ }^{152,210-212}$ an effect that can be assigned to conformational locking $^{152,212}$ or interlayer stabilization. ${ }^{210,213}$ Both modes of favorable hydrogen bonding are shown in Fig. 29. Still, one has to be careful not to decrease symmetry, as it has been observed that this can reduce both crystallinity and surface area. ${ }^{152,203,214}$ Additionally, partial linker fluorination can greatly improve both crystallinity and porosity as it induces strong phenyl-perfluorophenyl interactions. ${ }^{215-217}$

A completely different approach is based on the reduction of possible stacking modes. ${ }^{218-220}$ By intelligent choice of $\mathrm{COF}$ building blocks it is possible to induce geometrical constraints and by consequence increase crystallinity. This has been illustrated in Fig. 30, which shows "propeller" and "armchair" conformations on an example of TFPPy. Both conformations could induce specific stacking modes, reducing defects and thus increasing crystallinity. Moreover, the information can be transferred to neighboring building blocks in the same layer, if the appropriate linker is used. On the one hand, it was found that for "propeller" forming monomers, like ETTA, twisted building blocks were needed to impose correct steric limitations by phenylene tilt. ${ }^{219}$ On the other hand, TFPPy was experimentally found to prefer an "armchair" mode. Consequently, flat and rigid building blocks were needed to synchronize all monomers. ${ }^{218}$ Together, these effects impose serious limitations on the degrees of freedom of the system, resulting in increased crystallinity with domain sizes up to $500 \mathrm{~nm}$. Alternatively, strong dipole interactions can also induce such directed growth by the appearance of favorable stacking interactions. ${ }^{221}$ Another example of directional uniform growth inducing crystallinity has been considered in a theoretical study. ${ }^{222}$ Here monomer, dimer, trimer and hexamer monomers were compared. It was found that the hexamer building block, which can de facto be seen as one pore of a hcb net, tended to grow uniformly in all directions. Therefore, less defective 5- and 7-membered rings were formed and crystallinity was improved.

It has to be noted that all previously described effects are primarily based on interlayer stacking interactions and are thus less (or even not) valid for 3D COFs. The following observations can be extended to both $2 \mathrm{D}$ and $3 \mathrm{D}$ materials. For example, it has to be taken into account that when linker length is increased, the reaction time to obtain crystalline materials will also increase. ${ }^{103,138}$ Additionally, it might be advantageous to develop monomers which can perform self-condensation, as depicted in Fig. 31. ${ }^{223-225}$ Besides the guarantee of correct stoichiometry, the main advantage of this method is its surprising solvent adaptability. It has been shown that this method could be used to obtain highly crystalline Py-Py-COF, $\mathrm{A}_{2}-\mathrm{B}_{2}-$ $\mathrm{COF}$ and BBO-COF, not only in typical COF solvent mixtures (dioxane/mesitylene, $\mathrm{DCB} / n$-butanol) but also in a range of pure organic solvents (dichloromethane, tetrahydrofuran, ethanol, acetonitrile, etc.). This solvent adaptability was not observed for the related materials obtained by co-condensation and might 

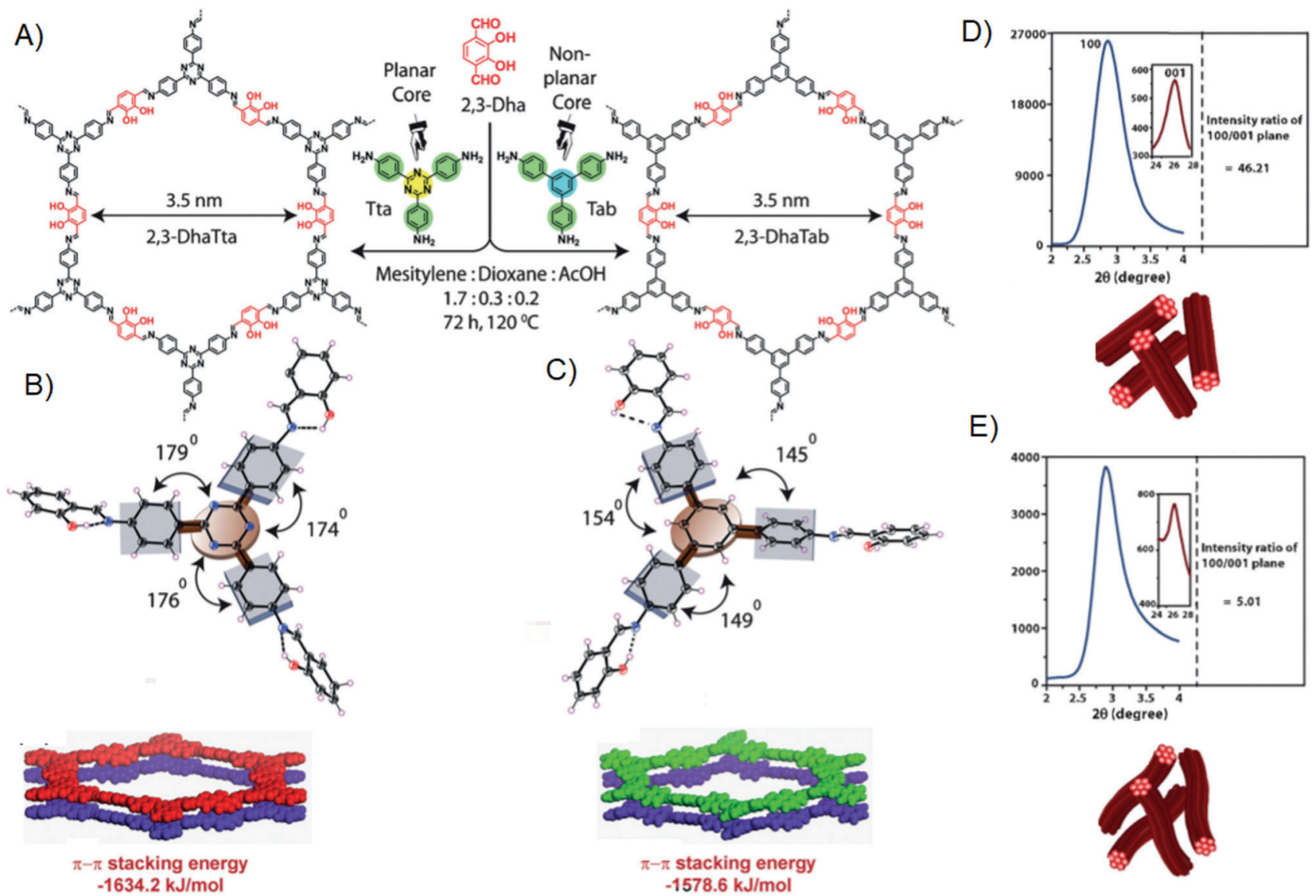

E)

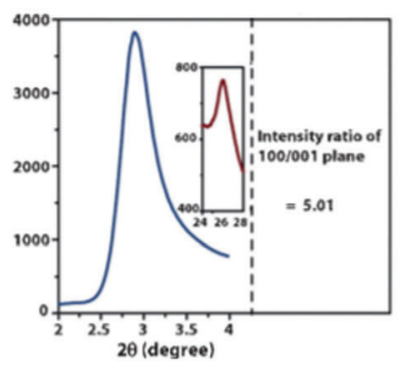

Fig. 28 (A) Schematic illustration of the synthesis of a planar (2,3-DhaTta) and non-planar (2,3-DhaTab) COF. (B and C) ORTEP diagrams of model compounds using planar (B) and non-planar (C) amine and the resulting $\pi-\pi$ stacking energy for the layers. (D and E) PXRD(100)/(001) intensity ratios for planar (D) and non-planar (E) amines and morphology of the obtained crystallites. Reproduced and adapted with permission from ref. 202, Copyright John Wiley and Sons (2016).

be related to the absence of need for matching linker solubilities. Moreover, materials quality was observed to be improved if selfcondensation was used, with sharper PXRD reflections and higher surface areas.

\subsection{Other methods}

Some techniques to prepare high-quality COFs could not be placed in one of the afore mentioned categories and will thus be discussed in the following.

For example, often used to increase reaction speed is microwave COF synthesis. Additionally, COF synthesis in microwaves allows continuous reaction monitoring and high controlability of reaction temperature and pressure, even in the center of reactions. ${ }^{226}$ Since the first report, microwave irradiation has been used to obtain COFs with a wide variety of linkage types (e.g. boroxine, ${ }^{226,227}$ imine, ${ }^{228} \beta$-ketoenamine, ${ }^{229}$ imide $^{230}$ ) in times as little as 20 minutes. Moreover, in some cases, the quality of the resulting material can be higher than solvothermally synthesized COFs. ${ }^{229}$ Similarly, a Parr reactor could be used to synthesize crystalline amide linked COFs. A pre-made amorphous amide polymer could be crystallized by three day reaction with 10 eq. $\mathrm{H}_{2} \mathrm{O}$ at $250{ }^{\circ} \mathrm{C}$ and $10^{-4}$ mbar in a water-containing Parr reactor. ${ }^{47}$
Another often used technique is (Liquid Assisted) Mechanochemical Grinding, which is simple, fast and both economically and ecologically attractive. ${ }^{231}$ This process can be performed completely solvent-free, ${ }^{231}$ or by addition of catalytic amounts of solvents. ${ }^{232}$ However, the obtained materials show inferior qualities with moderate crystallinity and low surface areas. $^{231-234}$

Additional to the techniques already mentioned in Section 3.4, high-quality COF thin films could be obtained by room temperature vapor deposition ${ }^{235}$ or growth on single layer graphene. $^{236}$

Finally, in some cases it was proven possible to obtain crystalline materials resulting from almost purely irreversible reactions. Those can be roughly divided in three categories: (1) materials formed by reversible linkages, that are subsequently transformed to an irreversible, more stable linkage. Which is technically a crystalline to crystalline transformation as the ones shown in Section 3.5.2 but without exchange of linkers. (2) Activation of monomers to achieve quasi-reversibility. (3) Decreasing the degrees of freedom, which has also been done (to a certain amount) to increase crystallinity of reversible linkages (as discussed in Section 3.6. A recent review by Haase and Lotsch covers this approach in much greater detail. ${ }^{237}$ 
<smiles>Oc1cc(/C=N/c2ccccc2)c(O)cc1/C=N/c1ccccc1</smiles>

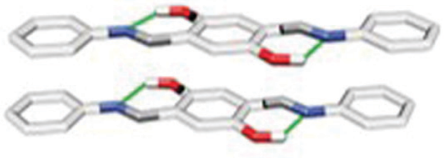

Conformational locking<smiles>[R]NC(=O)c1c(-c2ccccc2)c(C(=O)N[R])c(-c2ccccc2)c(C(=O)N[R])c1-c1ccccc1</smiles>

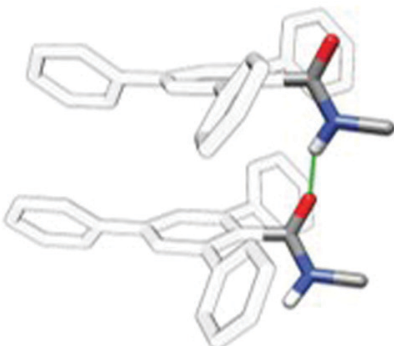

Interlayer stabilization

Fig. 29 Two modes of favorable hydrogen bonding in COF crystallization. Coplanar hydrogen bonding induces conformational locking of the imine bond, while intralayer hydrogen bonding induces strong interactions between the layers. Reproduced and adapted with permission from ref. 213, Copyright American Chemical Society (2020).
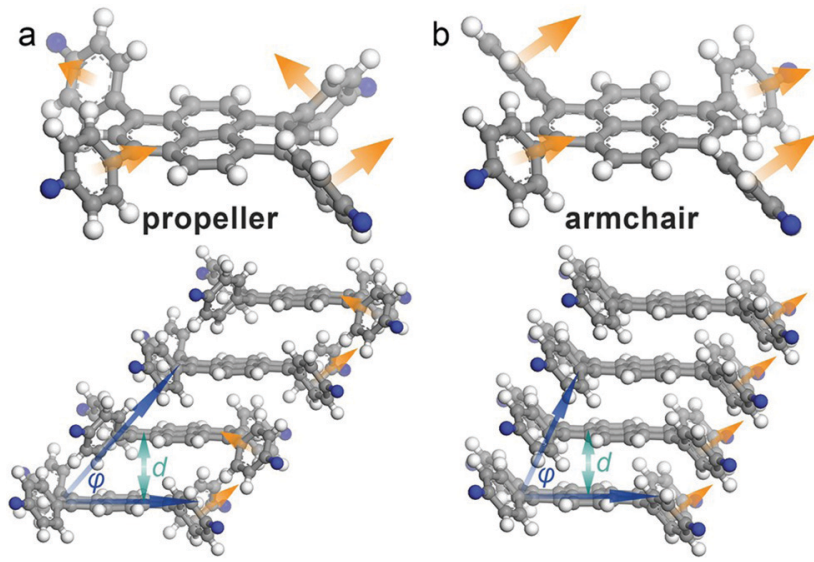

Fig. 30 COF building blocks imposing geometrical constraints when incorporated into COF (C, gray; $\mathrm{H}$, white; $\mathrm{N}$, blue), illustrated by TFPPy. Two conformations are shown: (A) propeller: the normal vectors of the phenylenes describe a circle, which induces offset stacks of alternated left- and right handed propellers. (B) Armchair: the normal vectors of the phenylenes point in the same direction, stacking in slip-stacked columns. Reproduced with permission from ref. 218, Copyright American Chemical Society (2016)

The most famous example of the combination of reversible and irreversible reactions are the $\beta$-ketoenamine TpPA- 1 and TpPA-2 COFs that were already extensively discussed before and is illustrated in Fig. 32A. ${ }^{35} \mathrm{~A}$ highly reversible imine condensation is formed by an irreversible keto-enol tautomerization to create a highly stable and highly crystalline material. Alternatively, COFs could be formed by multicomponent reactions, forming several covalent bonds at once via a combination of reversible reactions. This resulted in an imidazole COF formed<smiles>Nc1ccc(CCc2ccc(C=O)cc2)cc1CCc1ccc(C=O)cc1</smiles>

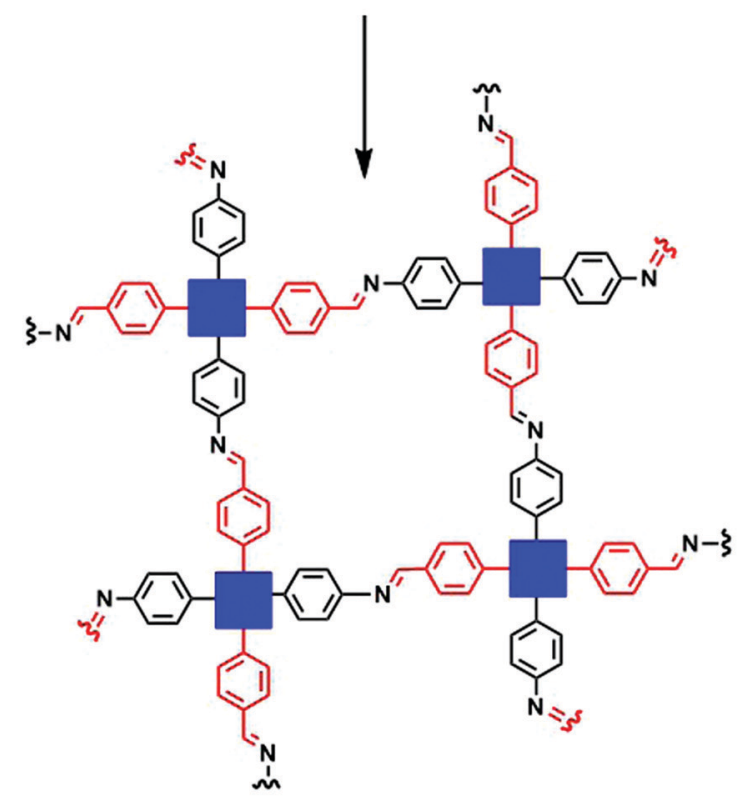

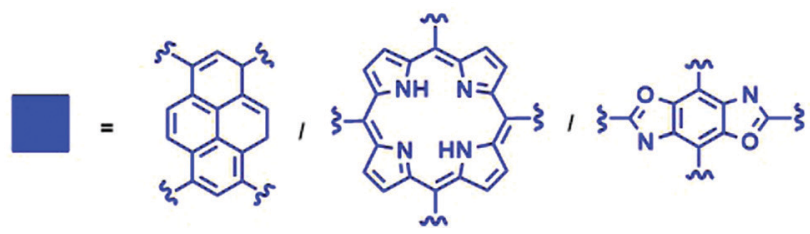

Fig. 31 Schematic representation of COF monomers capable of performing self-condensation. A monomer containing both amine and aldehyde functions is polymerized to an imine COF.

by the Debus-Radziszewski reaction, formation of this COF is schematically presented in Fig. 32B. ${ }^{238}$ Other examples of this approach include the post-modification of imine linkages to amide (as shown in Fig. 32C), ${ }^{48}$ carbamate, ${ }^{239}$ quinoline ${ }^{240}$ and thiazole. ${ }^{241,242}$ These post-synthetic linkage modifications have been excellently covered in a number of recent reviews. ${ }^{237,243}$

Monomer activation has been used for crystalline $\mathrm{C}=\mathrm{C}$ linked materials by Knoevenagel condensation. A cyanide functionality on the nucleophile stabilizes the intermediate carbon anion, resulting in partial reversibility. ${ }^{44,244}$ Alternatively, cyanide functionalized methylpyridines, ${ }^{245}$ methyltriazines ${ }^{50,51}$ or even in situ trimerized acetonitrile ${ }^{246}$ could be used.

Finally, crystalline COFs can be obtained via irreversible reactions if the used monomers are highly rigid. This decreases the risk of defect formation by linker misplacement, reducing the need for reversibility. Still, most often, additional synthetic improvements like slow monomer addition (as described in Section 3.2.2) are needed to obtain high-quality materials. ${ }^{237}$ 
A)<smiles>O=Cc1c(O)c(C=O)c(O)c(C=O)c1O</smiles>
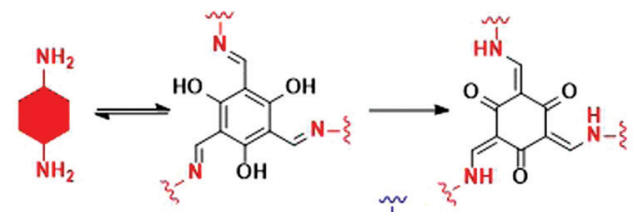

B)

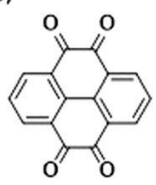

C)
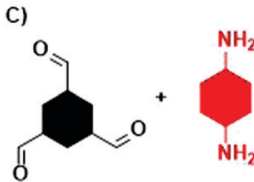

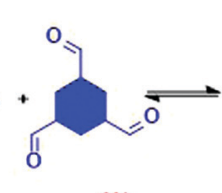

(1)

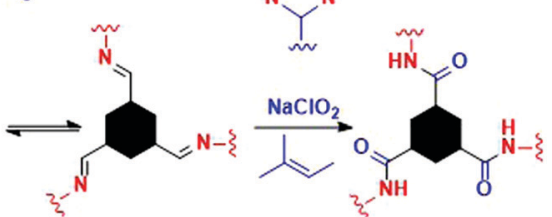

Fig. 32 Three different combinations of reversible and irreversible reactions to form COF. (A) $\beta$-Ketoenamine COFs are formed by a reversible imine condensation followed by an irreversible keto-enol transformation. ${ }^{35}$ (B) A highly stable imidazole linked COF is obtained by the simultaneous formation of different covalent bonds via reversible reactions. ${ }^{238}$ (C) An imine linked COF is post modified via an irreversible reaction to form a stable amide linkage. ${ }^{48}$

This way, crystalline COFs could be formed by Eglinton coupling of alkynes ${ }^{247}$ or debrominative Ullman coupling. ${ }^{248,249}$

Obviously, it is also possible to combine two or more of these approaches, for example dioxin-linked COF-316 and COF318 could be crystallized from highly rigid cyano activated nucleophile monomers. ${ }^{49}$ Similarly, vinylene linked COFs could be formed via Horner-Wadsworth-Emmons reaction, due to phosphonate intermediate stabilization and the use of rigid linkers. ${ }^{250}$

\section{Conclusions and outlook}

Since its first report in 2005, COFs have transformed from once precious, hard to reach compounds, to mature materials which can be used in a plethora of applications. This is mainly attributed to the high designability with more and more reported linkages crystallized in different underlying nets. Lately, it has even been proven possible to use highly stable linkages (like vinylene) for crystalline COFs. It is our expectation that COFs based on those quasi-irreversible, highly stable linkages will become more and more important as new linkage functionalities and more studies on existing functionalities will appear. Most likely, structural variety will be efficiently extended by the reticulation of previously inaccessible nets, as only a small fraction of all possibilities have already been explored. Combination of the extended library of linkages with the almost unlimited variety in nets will lead to readily obtainable tailor-made materials.

However, many challenges still have to be solved to reach this synthetic dream. Until now, most COF synthetic protocols are trial and error based and no generalized methods exist. Even a small variety in functional group or linker geometry can completely alter required synthetic conditions, leading to high

amounts of wasted time and effort in ever repeating synthetic optimization. Therefore, it is of vital importance to gather more knowledge on the formation of COFs. We have shown that while quite a lot is already known about boroxine and imine COFs, knowledge of other linkage types is superficial at best. Even for imine and boroxine linkages, a more in depth knowledge is required in order to be able to accurately predict condition and protocol feasibility. Ultimately, we expect the increased knowledge obtained by more in depth mechanistic studies on COF formation to allow higher control over materials quality, structure and properties while requiring less synthetic trials and effort.

Different approaches to achieve increased COF quality have been proposed and discussed extensively. It is our firm believe that each of these can greatly advance COF synthesis if applied correctly. For example, modulation has not been applied very often yet, but has proven to be a reliable method to improve both imine COF quality and crystallite size, with the appearance of single crystal COFs using a modulated approach. It would be very interesting to test its value for other, less reversible, linkages and more different materials. Additionally, control over monomer availability has already proven its worth in a larger library of COFs. Until now, physical delay is most straightforward and has yielded the best results. However, we expect chemical delay, by in situ linker generation, to play a pronounced role in 'real life' COF applications, due to its elegancy and simplicity. Possibly, synergetic, one-pot effects between linker generation and COF crystallization can be found. Huge catalytic improvements have already been made by the use of metal triflates or PTSA in imine COF synthesis. Still, most often, aqueous acetic acid is used, even though it is known not to be a very efficient catalyst. However, the most promising catalysts may even be far less studied. Despite only being very recently tested for COF synthesis, ILs and DESs have shown very promising results, obtaining highly crystalline materials in short reactions with mild conditions. Meanwhile, interfacial COF growth has proven its worth in the synthesis of highly crystalline COF thin films and might become the go to method for the preparation of these films. Finally, linker exchange can be convenient in order to prepare "hard to synthesize" COFs. Especially crystalline to crystalline exchange can be attractive as a COF can be made from a pre-made isoreticular material, reducing the need for correct network formation. Candidates for the "parent" material in such a transformation can be selected using the mentioned linker and condition optimization methods, selecting the ones guaranteed to yield the highest quality while being easy to exchange out.

Together, we expect that the presented approaches can be used as a toolbox to increase the quality of known COFs and make synthesis of new COFs more easy. While all have shown to be promising, practical use can often be highly situational. Modulation and decreased monomer availability can significantly increase crystallite size. But to achieve this, synthetic time is extended to weeks or months, making the obtained materials highly interesting for in-depth studies but useless for practical applications. Contrarily, improved catalysts and 
interfacial growth make it possible to obtain crystalline COFs rapidly, but seems not to increase crystallite size. Possibly, Ils and DESs might be able to combine the use of short reaction times with the obtainment of big, high-quality, crystallites as a completely different synthetic pathway is followed. Another option might be the combination of modulation with improved catalysts, to increase both reversibility and reaction efficiency.

In turn, this might increase the in depth knowledge of COFs as more detailed structural analysis using SXRD, HR-TEM or electron diffraction becomes possible. Structural knowledge gained from these advanced techniques might be very important to really understand COF chemistry and provide explanations for observed phenomena as pore blocking, layer shifting and interpenetration. Additionally, high-quality COFs can severely increase activity in applications as optoelectronics and photocatalysis.

\section{Conflicts of interest}

There are no conflicts to declare.

\section{Acknowledgements}

L. B. thanks Ghent University for funding. C. K. and P. V. D. V. acknowledge the Research Board of Ghent University (GOA01017, BOF GOA2017000303) for funding. K. V. H. thanks the Research Foundation - Flanders (FWO) (project G099319N) for funding.

\section{References}

1 A. P. Cote, A. I. Benin, N. W. Ockwig, M. O'Keeffe, A. J. Matzger and O. M. Yaghi, Science, 2005, 310, 1166-1170.

2 G. N. Lewis, J. Am. Chem. Soc., 1916, 38, 762-785.

3 T. Q. Ma, E. A. Kapustin, S. X. Yin, L. Liang, Z. Y. Zhou, J. Niu, L. H. Li, Y. Y. Wang, J. Su, J. Li, X. G. Wang, W. D. Wang, W. Wang, J. L. Sun and O. M. Yaghi, Science, 2018, 361, 48-52.

4 O. M. Yaghi, M. J. Kalmutzki and C. S. Diercks, Introduction to Reticular Chemistry, Wiley-VCH, Weinheim, 1st edn, 2019, pp. 179-195.

5 H. Staudinger, Ber. Dtsch. Chem. Ges. (A and B Series), 1920, 53, 1073-1085.

6 H. Frey and T. Johann, Polym. Chem., 2020, 11, 8-14.

7 F. Wöhler, Ann. Phys. Chem., 1828, 2, 253-256.

8 W. H. Perkin, Producing a New Coloring Matter for Dyeing with a Lilac or Purple Color Stuffs of Silk, Cotton, Wool, or other Materials, 1856.

9 H. Dreser, Arch. Gesamte Physiol. Menschen Tiere, 1899, 76, 306-318.

10 K. C. Nicolaou, Proc. R. Soc. London, Ser. A, 2014, 470, 20130690.
11 O. T. Benfey and P. J. T. Morris, Robert Burns Woodward: Architect and Artist in the World of Molecules, 1st edn, Chemical Heritage Foundation, Philadelphia, 2001.

12 R. B. Woodward, Pure Appl. Chem., 1973, 33, 145-178.

13 R. Hoffmann, Sci. Am., 1993, 268, 66-73.

14 C. Pedersen, J. Am. Chem. Soc., 1967, 89, 7017-7036.

15 B. Dietrich, J. M. Lehn, J. P. Sauvage and J. Blanzat, Tetrahedron, 1973, 29, 1629-1645.

16 W. Kiggen and F. Vögtle, Angew. Chem., Int. Ed. Engl., 1984, 23, 714-715.

17 D. J. Cram, S. Karbach, Y. H. Kim, L. Baczynskyj and G. W. Kallemeyn, J. Am. Chem. Soc., 1985, 107, 2575-2576.

18 S. J. Rowan, S. J. Cantrill, G. R. L. Cousins, J. K. M. Sanders and J. F. Stoddart, Angew. Chem., Int. Ed., 2002, 41, 898-952.

19 C. Dietrich-Buchecker, J. Sauvage and J. Kintzinger, Tetrahedron Lett., 1983, 24, 5095-5098.

20 K. S. Chichak, S. J. Cantrill, A. R. Pease, S.-H. Chiu, G. W. V. Cave, J. L. Atwood and J. F. Stoddart, Science, 2004, 304, 1308-1312.

21 M. L. C. Quan and D. J. Cram, J. Am. Chem. Soc., 1991, 113, 2754-2755.

22 G. Zhang and M. Mastalerz, Chem. Soc. Rev., 2014, 43, 1934-1947.

23 H. M. El-Kaderi, J. R. Hunt, J. L. Mendoza-Cortés, A. P. Côté, R. E. Taylor, M. O'Keeffe and O. M. Yaghi, Science, 2007, 316, 268-272.

24 Y.-B. Zhang, J. Su, H. Furukawa, D. F. G. Yifeng Yun, A. Duong, X. Zou and O. M. Yaghi, J. Am. Chem. Soc., 2013, 135, 16336-16339.

25 D. Beaudoin, T. Maris and J. Wuest, Nat. Chem., 2013, 5, 830-834.

26 Y. Liu, Y. Ma, Y. Zhao, X. Sun, F. Gándara, H. Furukawa, Z. Liu, H. Zhu, C. Zhu, K. Suenaga, P. Oleynikov, A. S. Alshammari, X. Zhang, O. Terasaki and O. M. Yaghi, Science, 2016, 351, 365-369.

27 X. Chen, K. Geng, R. Liu, K. T. Tan, Y. Gong, Z. Li, S. Tao, Q. Jiang and D. Jiang, Angew. Chem., Int. Ed., 2020, 59, 5050-5091.

28 J. L. Segura, S. Royuela and M. M. Ramos, Chem. Soc. Rev., 2019, 48, 3903-3945.

29 O. M. Yaghi, M. J. Kalmutzki and C. S. Diercks, Introduction to Reticular Chemistry, Wiley-VCH, Weinheim, 1st edn, 2019, pp. 197-223.

30 J. R. Hunt, C. J. Doonan, J. D. LeVangie, A. P. Côté and O. M. Yaghi, J. Am. Chem. Soc., 2008, 130, 11872-11873.

31 P. Kuhn, M. Antonietti and A. Thomas, Angew. Chem., Int. Ed., 2008, 47, 3450-3453.

32 F. J. Uribe-Romo, J. R. Hunt, H. Furukawa, C. Klöck, M. OKeeffe and O. M. Yaghi, J. Am. Chem. Soc., 2009, 131, 4570-4571.

33 J. L. Segura, M. J. Mancheño and F. Zamora, Chem. Soc. Rev., 2016, 45, 5635-5671.

34 F. J. Uribe-Romo, C. J. Doonan, H. Furukawa, K. Oisaki and O. M. Yaghi, J. Am. Chem. Soc., 2011, 133, 11478-11481.

35 S. Kandambeth, A. Mallick, B. Lukose, M. V. Mane, T. Heine and R. Banerjee, J. Am. Chem. Soc., 2012, 134, 19524-19527. 
36 J. Guo, Y. Xu, S. Jin, L. Chen, T. Kaji, Y. Honsho, M. A. Addicoat, J. Kim, A. Saeki and H. Ihee, et al., Nat. Commun., 2013, 4, 2736.

37 A. Nagai, X. Chen, X. Feng, X. Ding, Z. Guo and D. Jiang, Angew. Chem., Int. Ed., 2013, 52, 3770-3774.

38 D. A. Pyles, J. W. Crowe, L. A. Baldwin and P. L. McGrier, ACS Macro Lett., 2016, 5, 1055-1058.

39 S.-Y. Jiang, S.-X. Gan, X. Zhang, H. Li, Q.-Y. Qi, F.-Z. Cui, J. Lu and X. Zhao, J. Am. Chem. Soc., 2019, 141, 14981-14986.

40 H. Zhao, Z. Jin, H. Su, X. Jing, F. Sun and G. Zhu, Chem. Commun., 2011, 47, 6389-6391.

41 K. T. Jackson, T. E. Reich and H. M. El-Kaderi, Chem. Commun., 2012, 48, 8823-8825.

42 Q. Fang, Z. Zhuang, S. Gu, R. B. Kaspar, J. Zheng, J. Wang, S. Qiu and Y. Yan, Nat. Commun., 2014, 5, 4503.

43 Y. Du, H. Yang, J. M. Whiteley, S. Wan, Y. Jin, S. Lee and W. Zhang, Angew. Chem., Int. Ed., 2015, 55, 1737-1741.

44 X. Zhuang, W. Zhao, F. Zhang, Y. Cao, F. Liu, S. Bi and X. Feng, Polym. Chem., 2016, 7, 4176-4181.

45 E. Jin, J. Li, K. Geng, Q. Jiang, H. Xu, Q. Xu and D. Jiang, Nat. Commun., 2018, 9, 4143.

46 J. Roeser, D. Prill, M. J. Bojdys, P. Fayon, A. Trewin, A. N. Fitch, M. U. Schmidt and A. Thomas, Nat. Chem., 2017, 9, 977-982.

47 D. Stewart, D. Antypov, M. S. Dyer, M. J. Pitcher, A. P. Katsoulidis, P. A. Chater, F. Blanc and M. J. Rosseinsky, Nat. Commun., 2017, 8, 1102.

48 P. J. Waller, S. J. Lyle, T. M. Osborn Popp, C. S. Diercks, J. A. Reimer and O. M. Yaghi, J. Am. Chem. Soc., 2016, 138, 15519-15522.

49 B. Zhang, M. Wei, H. Mao, X. Pei, S. A. Alshmimri, J. A. Reimer and O. M. Yaghi, J. Am. Chem. Soc., 2018, 140, 12715-12719.

50 H. Lyu, C. S. Diercks, C. Zhu and O. M. Yaghi, J. Am. Chem. Soc., 2019, 141, 6848-6852.

51 A. Acharjya, P. Pachfule, J. Roeser, F.-J. Schmitt and A. Thomas, Angew. Chem., Int. Ed., 2019, 58, 14865-14870.

52 K. C. Ranjeesh, R. Illathvalappil, S. D. Veer, J. Peter, V. C. Wakchaure, Goudappagouda, K. V. Raj, S. Kurungot and S. S. Babu, J. Am. Chem. Soc., 2019, 141, 14950-14954.

53 C. Zhao, H. Lyu, Z. Ji, C. Zhu and O. M. Yaghi, J. Am. Chem. Soc., 2020, 142, 14450-14454.

54 J. Liu, T. Yang, Z.-P. Wang, P.-L. Wang, J. Feng, S.-Y. Ding and W. Wang, J. Am. Chem. Soc., 2020, 142, 20956-20961.

55 C. Gropp, T. Ma, N. Hanikel and O. M. Yaghi, Science, 2020, 370, eabd6406.

56 C. Gropp, S. Canossa, S. Wuttke, F. Gándara, Q. Li, L. Gagliardi and O. M. Yaghi, ACS Cent. Sci., 2020, 6, 1255-1273.

57 S. Dalapati, S. Jin, J. Gao, Y. Xu, A. Nagai and D. Jiang, J. Am. Chem. Soc., 2013, 135, 17310-17313.

58 G. Das, B. P. Biswal, S. Kandambeth, V. Venkatesh, G. Kaur, M. Addicoat, T. Heine, S. Verma and R. Banerjee, Chem. Sci., 2015, 6, 3931-3939.

59 C. Zhao, C. S. Diercks, C. Zhu, N. Hanikel, X. Pei and O. M. Yaghi, J. Am. Chem. Soc., 2018, 140, 16438-16441.
60 M. J. Bojdys, S. A. Wohlgemuth, A. Thomas and M. Antonietti, Macromolecules, 2010, 43, 6639-6645.

61 M. R. Rao, Y. Fang, S. De Feyter and D. F. Perepichka, J. Am. Chem. Soc., 2017, 139, 2421-2427.

62 A. F. Wells, Acta Crystallogr., 1954, 7, 535-544.

63 O. M. Yaghi, M. O'Keeffe, N. W. Ockwig, H. K. Chae, M. Eddaoudi and J. Kim, Nature, 2003, 423, 705-714.

64 M. OKeeffe, M. A. Peskov, S. J. Ramsden and O. M. Yaghi, Acc. Chem. Res., 2008, 41, 1782-1789.

65 O. M. Yaghi, M. J. Kalmutzki and C. S. Diercks, Introduction to Reticular Chemistry, 1st edn, Wiley-VCH, Weinheim, 2019, pp. 225-243.

66 O. M. Yaghi, M. J. Kalmutzki and C. S. Diercks, Introduction to Reticular Chemistry, 1st edn, Wiley-VCH, Weinheim, 2019, pp. 431-452.

67 N. W. Ockwig, O. Delgado-Friedrichs, M. O'Keeffe and O. M. Yaghi, Acc. Chem. Res., 2005, 38, 176-182.

68 S.-Q. Xu, R.-R. Liang, T.-G. Zhan, Q.-Y. Qi and X. Zhao, Chem. Commun., 2017, 53, 2431-2434.

69 S. Wan, F. Gándara, A. Asano, H. Furukawa, A. Saeki, S. K. Dey, L. Liao, M. W. Ambrogio, Y. Y. Botros, X. Duan, S. Seki, J. F. Stoddart and O. M. Yaghi, Chem. Mater., 2011, 23, 4094-4097.

70 S. Dalapati, M. Addicoat, S. Jin, T. Sakurai, J. Gao, H. Xu, S. Irle, S. Seki and D. Jiang, Nat. Commun., 2015, 6, 7786.

71 T.-Y. Zhou, S.-Q. Xu, Q. Wen, Z.-F. Pang and X. Zhao, J. Am. Chem. Soc., 2014, 136, 15885-15888.

72 H. L. Nguyen, N. Hanikel, S. J. Lyle, C. Zhu, D. M. Proserpio and O. M. Yaghi, J. Am. Chem. Soc., 2020, 142, 2218-2221.

73 C. Qian, Q.-Y. Qi, G.-F. Jiang, F.-Z. Cui, Y. Tian and X. Zhao, J. Am. Chem. Soc., 2017, 139, 6736-6743.

74 Z.-J. Yin, S.-Q. Xu, T.-G. Zhan, Q.-Y. Qi, Z. Quan and X. Zhao, Chem. Commun., 2017, 53, 7266-7269.

75 T. Banerjee, F. Haase, S. Trenker, B. P. Biswal, G. Savasci, V. Duppel, I. Moudrakovski, C. Ochsenfeld and B. V. Lotsch, Nat. Commun., 2019, 10, 2689.

76 H. L. Nguyen, C. Gropp and O. M. Yaghi, J. Am. Chem. Soc., 2020, 142, 2771-2776.

77 B. Zhang, H. Mao, R. Matheu, J. A. Reimer, S. A. Alshmimri, S. Alshihri and O. M. Yaghi, J. Am. Chem. Soc., 2019, 141, 11420-11424.

78 S. J. Lyle, P. J. Waller and O. M. Yaghi, Trends Chem., 2019, 1, 172-184.

79 X. Guan, F. Chen, Q. Fang and S. Qiu, Chem. Soc. Rev., 2020, 49, 1357-1384.

80 G. Lin, H. Ding, D. Yuan, B. Wang and C. Wang, J. Am. Chem. Soc., 2016, 138, 3302-3305.

81 Y. Zhang, J. Duan, D. Ma, P. Li, S. Li, H. Li, J. Z. Xiaojie Ma, X. Feng and B. Wang, Angew. Chem., Int. Ed., 2017, 56, 16313-16317.

82 Y. Liu, C. S. Diercks, Y. Ma, H. Lyu, C. Zhu, S. A. Alshmimri, S. Alshihri and O. M. Yaghi, J. Am. Chem. Soc., 2019, 141, 677-683.

83 Y. Zhao, L. Guo, F. Gándara, Y. Ma, Z. Liu, C. Zhu, H. Lyu, C. A. Trickett, E. A. Kapustin, O. Terasaki and O. M. Yaghi, J. Am. Chem. Soc., 2017, 139, 13166-13172. 
84 O. Yahiaoui, A. N. Fitch, F. Hoffmann, M. Fröba, A. Thomas and J. Roeser, J. Am. Chem. Soc., 2018, 140, 5330-5333.

85 Y. Lan, X. Han, M. Tong, H. Huang, Q. Yang, D. Liu, X. Zhao and C. Zhong, Nat. Commun., 2018, 9, 5274.

86 X. Kang, X. Han, C. Yuan, C. Cheng, Y. Liu and Y. Cui, J. Am. Chem. Soc., 2020, 142, 16346-16356.

87 H. L. Nguyen, C. Gropp, Y. Ma, C. Zhu and O. M. Yaghi, J. Am. Chem. Soc., 2020, 142, 20335-20339.

88 H. Li, J. Ding, X. Guan, F. Chen, C. Li, L. Zhu, M. Xue, D. Yuan, V. Valtchev, Y. Yan, S. Qiu and Q. Fang, J. Am. Chem. Soc., 2020, 142, 13334-13338.

89 Q. Zhu, X. Wang, R. Clowes, P. Cui, L. Chen, M. A. Little and A. I. Cooper, J. Am. Chem. Soc., 2020, 142, 16842-16848.

90 H. Li, F. Chen, X. Guan, J. Li, C. Li, B. Tang, V. Valtchev, Y. Yan, S. Qiu and Q. Fang, J. Am. Chem. Soc., 2021, 143, 2654-2659.

91 Z. Li, L. Sheng, H. Wang, X. Wang, M. Li, Y. Xu, H. Cui, H. Zhang, H. Liang, H. Xu and X. He, J. Am. Chem. Soc., 2021, 143(1), 92-96.

92 B. Lukose, A. Kuc and T. Heine, Chem. - Eur. J., 2011, 17, 2388-2392.

93 A. Mähringer and D. D. Medina, Nat. Chem., 2020, 12, 985-987.

94 Y. Du, D. Calabro, B. Wooler, Q. Li, S. Cundy, P. Kamakoti, D. Colmyer, K. Mao and P. Ravikovitch, J. Phys. Chem. C, 2014, 118, 399-407.

95 X. Wu, X. Han, Y. Liu, Y. Liu and Y. Cui, J. Am. Chem. Soc., 2018, 140, 16124-16133.

96 Y. Zhu, S. Wan, Y. Jin and W. Zhang, J. Am. Chem. Soc., 2015, 137, 13772-13775.

97 S.-L. Cai, K. Zhang, J.-B. Tan, S. Wang, S.-R. Zheng, J. Fan, Y. Yu, W.-G. Zhang and Y. Liu, ACS Macro Lett., 2016, 5, 1348-1352.

98 L. Zhu and Y.-B. Zhang, Molecules, 2017, 22, 1149.

99 J. Jiang, Y. Zhao and O. M. Yaghi, J. Am. Chem. Soc., 2016, 138, 3255-3265.

100 P. J. Waller, F. Gándara and O. M. Yaghi, Acc. Chem. Res., 2015, 48, 3053-3063.

101 X. Ma and T. F. Scott, Chem. Commun., 2018, 1, 98.

102 B. J. Smith and W. R. Dichtel, J. Am. Chem. Soc., 2014, 136, 8783-8789.

103 B. J. Smith, N. Hwang, A. D. Chavez, J. L. Novotney and W. R. Dichtel, Chem. Commun., 2015, 51, 7532-7535.

104 A. M. Evans, L. R. Parent, N. C. Flanders, R. P. Bisbey, E. Vitaku, M. S. Kirschner, R. D. Schaller, L. X. Chen, N. C. Gianneschi and W. R. Dichtel, Science, 2018, 361, 52-57.

105 S. Clair, M. Abel and L. Porte, Chem. Commun., 2014, 50, 9627-9635.

106 H. Li, A. D. Chavez, H. Li, H. Li, W. R. Dichtel and J.-L. Bredas, J. Am. Chem. Soc., 2017, 139, 16310-16318.

107 B. T. Koo, R. F. Heden and P. Clancy, Phys. Chem. Chem. Phys., 2017, 19, 9745-9754.

108 V. Nguyen and M. Grünwald, J. Am. Chem. Soc., 2018, 140, 3306-3311.
109 B. J. Smith, L. R. Parent, A. C. Overholts, P. A. Beaucage, R. P. Bisbey, A. D. Chavez, N. Hwang, C. Park, A. M. Evans, N. C. Gianneschi and W. R. Dichtel, ACS Cent. Sci., 2017, 3, 58-65.

110 A. M. Evans, I. Castano, A. Brumberg, L. R. Parent, A. R. Corcos, R. L. Li, N. C. Flanders, D. J. Gosztola, N. C. Gianneschi, R. D. Schaller and W. R. Dichtel, J. Am. Chem. Soc., 2019, 141, 19728-19735.

111 H. Li, A. M. Evans, I. Castano, M. J. Strauss, W. R. Dichtel and J.-L. Bredas, J. Am. Chem. Soc., 2020, 142, 1367-1374.

112 B. J. Smith, A. C. Overholts, N. Hwang and W. R. Dichtel, Chem. Commun., 2016, 52, 3690-3693.

113 C. H. Feriante, S. Jhulki, A. M. Evans, R. R. Dasari, K. Slicker, W. R. Dichtel and S. R. Marder, Adv. Mater., 2020, 32, 1905776.

114 M. E. Belowich and J. F. Stoddart, Chem. Soc. Rev., 2012, 41, 2003-2024.

115 P. G. Cozzi, Chem. Soc. Rev., 2004, 33, 410-421.

116 M. Ciaccia and S. Di Stefano, Org. Biomol. Chem., 2015, 13, 646-654.

117 J. Tan, S. Namuangruk, W. Kong, N. Kungwan, J. Guo and C. Wang, Angew. Chem., Int. Ed., 2016, 55, 13979-13984.

118 C. Feriante, A. M. Evans, S. Jhulki, I. Castano, M. J. Strauss, S. Barlow, W. R. Dichtel and S. R. Marder, J. Am. Chem. Soc., 2020, 142, 18637-18644.

119 T. Sick, J. M. Rotter, S. Reuter, S. Kandambeth, N. N. Bach, M. Döblinger, J. Merz, T. Clark, T. B. Marder, T. Bein and D. D. Medina, J. Am. Chem. Soc., 2019, 141, 12570-12581.

120 D. Zhu and R. Verduzco, ACS Appl. Mater. Interfaces, 2020, 12, 33121-33127.

121 D. M. Fischbach, G. Rhoades, C. Espy, F. Goldberg and B. J. Smith, Chem. Commun., 2019, 55, 3594-3597.

122 L. Liang, Y. Qiu, W. D. Wang, J. Han, Y. Luo, W. Yu, G.-L. Yin, Z.-P. Wang, L. Zhang, J. Ni, J. Niu, J. Sun, T. Ma and W. Wang, Angew. Chem., Int. Ed., 2020, 59, 17991-17995.

123 L. Zhang, K. Zhao, H. Li, T. Zhang, D. Liu and Y. Han, J. Polym. Sci., Part B: Polym. Phys., 2019, 57, 1572-1591.

124 K. Gu and Y. Loo, J. Polym. Sci., Part B: Polym. Phys., 2019, 57, 1559-1571.

125 Z. Bao, A. Dodabalapur and A. Lovinger, Appl. Phys. Lett., 1996, 69, 4108.

126 I. McCulloch, M. Heeney, C. Bailey, K. Genevicius, I. MacDonald, M. Shkunov, D. Sparrowe, S. Tierney, R. Wagner, W. Zhang, M. Chabinyc, R. Kline, M. McGehee and M. Toney, Nat. Mater., 2006, 5, 328-333.

127 S. Thomas, H. Li, C. Zhong, M. Matsumoto, W. R. Dichtel and J.-L. Bredas, Chem. Mater., 2019, 31, 3051-3065.

128 Y. Jing and T. Heine, J. Am. Chem. Soc., 2019, 141, 743-747.

129 R. Gutzler and D. F. Perepichka, J. Am. Chem. Soc., 2013, 135, 16585-16594.

130 R. Gutzler, Phys. Chem. Chem. Phys., 2016, 18, 29092-29100. 131 A. Zhao and S.-Q. Shen, Phys. Rev. B: Condens. Matter Mater. Phys., 2012, 85, 085209.

132 N. B. Kopnin, T. T. Heikkilä and G. E. Volovik, Phys. Rev. B: Condens. Matter Mater. Phys., 2011, 83, 220503. 
133 G. Galeotti, F. De Marchi, E. Hamzehpoor, O. MacLean, M. Rajeswara Rao, Y. Chen, L. V. Besteiro, D. Dettmann, L. Ferrari, F. Frezza, P. M. Sheverdyaeva, R. Liu, A. K. Kundu, P. Moras, M. Ebrahimi, M. C. Gallagher, F. Rosei, D. F. Perepichka and G. Contini, Nat. Mater., 2020, 19, 874-880.

134 S. Zhu and D. Wang, Adv. Energy Mater., 2017, 7, 1700841.

135 K. Maeda, ACS Catal., 2013, 3, 1486-1503.

136 U. Diebold, Nat. Chem., 2011, 3, 271-272.

137 L. Lin, H. Ou, Y. Zhang and X. Wang, ACS Catal., 2016, 6, 3921-3931.

138 P. Pachfule, A. Acharjya, J. Roeser, T. Langenhahn, M. Schwarze, R. Schomäcker, A. Thomas and J. Schmidt, J. Am. Chem. Soc., 2018, 140, 1423-1427.

139 C. Krishnaraj, H. S. Jena, L. Bourda, A. Laemont, P. Pachfule, J. Roeser, C. V. Chandran, S. Borgmans, S. M. J. Rogge, K. Leus, C. V. Stevens, J. A. Martens, V. Van Speybroeck, E. Breynaert, A. Thomas and P. Van Der Voort, J. Am. Chem. Soc., 2020, 142, 20107-20116.

140 P.-F. Wei, M.-Z. Qi, Z.-P. Wang, S.-Y. Ding, W. Yu, Q. Liu, L.-K. Wang, H.-Z. Wang, W.-K. An and W. Wang, J. Am. Chem. Soc., 2018, 140, 4623-4631.

141 C. Krishnaraj, H. S. Jena, K. Leus and P. Van Der Voort, Green Chem., 2020, 22, 1038-1071.

142 W. I. F. David and K. Shankland, Acta Crystallogr., Sect. A: Found. Crystallogr., 2008, 64, 52-64.

143 R. H. Blessing, Acta Crystallogr., Sect. A: Found. Adv., 2014, 70, 518-519.

144 F. Gándara and T. D. Bennett, IUCrJ, 2014, 1, 563-570.

145 F. T. L. Muniz, M. A. R. Miranda, C. Morilla dos Santos and J. M. Sasaki, Acta Crystallogr., Sect. A: Found. Adv., 2016, 72, 385-390.

146 S. Kavesh and J. M. Schultz, Polym. Eng. Sci., 1969, 9, 331-338.

147 T. Sun, L. Wei, Y. Chen, Y. Ma and Y.-B. Zhang, J. Am. Chem. Soc., 2019, 141, 10962-10966.

148 C. Gao, J. Li, S. Yin, G. Lin, T. Ma, Y. Meng, J. Sun and C. Wang, Angew. Chem., Int. Ed., 2019, 58, 9770-9775.

149 R. S. Forgan, Chem. Sci., 2020, 11, 4546-4562.

150 I. Castano, A. M. Evans, H. Li, E. Vitaku, M. J. Strauss, J.-L. Brédas, N. C. Gianneschi and W. R. Dichtel, ACS Cent. Sci., 2019, 5, 1892-1899.

151 M. Calik, T. Sick, M. Dogru, M. Döblinger, S. Datz, H. Budde, A. Hartschuh, F. Auras and T. Bein, J. Am. Chem. Soc., 2016, 138, 1234-1239.

152 R. A. Maia, F. L. Oliveira, M. Nazarkovsky and P. M. Esteves, Cryst. Growth Des., 2018, 18, 5682-5689.

153 W. Zhao, J. Qiao, T. Ning and X. Liu, Chinese J. Polym. Sci., 2018, 36, 1-7.

154 S. Wang, Z. Zhang, H. Zhang, A. G. Rajan, N. Xu, Y. Yang, Y. Zeng, P. Liu, X. Zhang, Q. Mao, Y. He, J. Zhao, B.-G. Li, M. S. Strano and W.-J. Wang, Matter, 2019, 1, 1592-1605.

155 D. Zhu, L. B. Alemany, W. Guo and R. Verduzco, Polym. Chem., 2020, 11, 4464-4468.

156 X. Han, J. Zhang, J. Huang, X. Wu, D. Yuan, Y. Liu and Y. Cui, Nat. Commun., 2018, 9, 1294.
157 X. Chen, L. Xia, R. Pan and X. Liu, J. Colloid Interface Sci., 2020, 568, 76-80.

158 W. Liu, X. Li, C. Wang, H. Pan, W. Liu, K. Wang, Q. Zeng, R. Wang and J. Jiang, J. Am. Chem. Soc., 2019, 141, 17431-17440.

159 Y. Liu, Y. Wang, H. Li, X. Guan, L. Zhu, M. Xue, Y. Yan, V. Valtchev, S. Qiu and Q. Fang, Chem. Sci., 2019, 10, 10815-10820.

160 E. L. Spitler and W. R. Dichtel, Nat. Chem., 2010, 2, 672-677.

161 E. L. Spitler, M. R. Giovino, S. L. White and W. R. Dichtel, Chem. Sci., 2011, 2, 1588-1593.

162 M. Dogru, A. Sonnauer, S. Zimdars, M. Döblinger, P. Knochel and T. Bein, CrystEngComm, 2013, 15, 1500-1502.

163 Z.-J. Li, S.-Y. Ding, H.-D. Xue, W. Cao and W. Wang, Chem. Commun., 2016, 52, 7217-7220.

164 M. Liu, Q. Huang, S. Wang, Z. Li, B. Li, S. Jin and B. Tan, Angew. Chem., Int. Ed., 2018, 57, 11968-11972.

165 Greene's Protective Groups in Organic Synthesis, 5th edn, ed. P. G. M. Wuts, John Wiley \& Sons, New York, 5th edn, 2014.

166 E. Vitaku and W. R. Dichtel, J. Am. Chem. Soc., 2017, 139, 12911-12914.

167 C. R. DeBlase, K. Hernández-Burgos, K. E. Silberstein, G. G. Rodríguez-Calero, R. P. Bisbey, H. D. Abruña and W. R. Dichtel, ACS Nano, 2015, 9, 3178-3183.

168 M. Liu, K. Jiang, X. Ding, S. Wang, C. Zhang, J. Liu, Z. Zhan, G. Cheng, B. Li, H. Chen, S. Jin and B. Tan, Adv. Mater., 2019, 31, 1807865.

169 D. Rodríguez-San-Miguel, A. Abrishamkar, J. A. R. Navarro, R. Rodriguez-Trujillo, D. B. Amabilino, R. Mas-Ballesté, F. Zamora and J. Puigmartí-Luis, Chem. Commun., 2016, 52, 9212-9215.

170 R. Li, N. C. Flanders, A. M. Evans, W. Ji, I. Castano, L. X. Chen, N. C. Gianneschi and W. R. Dichtel, Chem. Sci., 2019, 10, 3796-3801.

171 T. P. Vaid, S. P. Kelley and R. D. Rogers, IUCrJ, 2017, 4, 380-392.

172 X. Guan, Y. Ma, H. Li, Y. Yusran, M. Xue, Q. Fang, Y. Yan, V. Valtchev and S. Qiu, J. Am. Chem. Soc., 2018, 140, 4494-4498.

173 Y. Gao, C. Wang, H. Hu, R. Ge, M. Lu, J. Zhang, Z. Li, P. Shao and D. Jiang, Chem. - Eur. J., 2019, 25, 15488-15492.

174 P. Guan, J. Qiu, Y. Zhao, H. Wang, Z. Li, Y. Shi and J. Wang, Chem. Commun., 2019, 55, 12459-12462.

175 J. Qiu, H. Wang, Y. Zhao, P. Guan, Z. Li, H. Zhang, H. Gao, S. Zhang and J. Wang, Green Chem., 2020, 22, 2605-2612.

176 J. Qiu, P. Guan, Y. Zhao, Z. Li, H. Wang and J. Wang, Green Chem., 2020, 22, 7537-7542.

177 M. Matsumoto, R. R. Dasari, W. Ji, C. H. Feriante, T. C. Parker, S. R. Marder and W. R. Dichtel, J. Am. Chem. Soc., 2017, 139, 4999-5002.

178 N. Giuseppone, J.-L. Schmitt and J.-M. Lehn, Angew. Chem., Int. Ed., 2004, 43, 4902-4906.

179 M. Matsumoto, L. Valentino, G. M. Stiehl, H. B. Balch, A. R. Corcos, F. Wang, D. C. Ralph, B. J. Mariñas and W. R. Dichtel, Chem, 2018, 4, 308-317. 
180 H.-L. Qian, F.-L. Meng, C.-X. Yang and X.-P. Yan, Angew. Chem., Int. Ed., 2020, 59, 17607-17613.

181 C. Li, Y. Ma, H. Liu, L. Tao, Y. Ren, X. Chen, H. Li and Q. Yang, Chin. J. Catal., 2020, 41, 1288-1297.

182 S. Karak, S. Kandambeth, B. P. Biswal, H. S. Sasmal, S. Kumar, P. Pachfule and R. Banerjee, J. Am. Chem. Soc., 2017, 139, 1856-1862.

183 S. Karak, S. Kumar, P. Pachfule and R. Banerjee, J. Am. Chem. Soc., 2018, 140, 5138-5145.

184 S. Ren, M. J. Bojdys, R. Dawson, A. Laybourn, Y. Z. Khimyak, D. J. Adams and A. I. Cooper, Adv. Mater., 2012, 24, 2357-2361.

185 J. Maschita, T. Banerjee, G. Savasci, F. Haase, C. Ochsenfeld and B. V. Lotsch, Angew. Chem., Int. Ed., 2020, 59, 15750-15758.

186 L. Zhang, R. Liang, C. Hang, H. Wang, L. Sun, L. Xu, D. Liu, Z. Zhang, X. Zhang, F. Chang, S. Zhao and W. Huang, Green Chem., 2020, 22, 2498-2504.

187 F. Zhang, J. Zhang, B. Zhang, X. Tan, D. Shao, J. Shi, D. Tan, L. Liu, J. Feng, B. Han, G. Yang, L. Zheng and J. Zhang, ChemSusChem, 2018, 11, 3576-3580.

188 Y.-L. Zhu, H.-Y. Zhao, C.-L. Fu, Z.-W. Li, Z.-Y. Sun and Z. Lu, J. Phys. Chem. Lett., 2020, 11, 9952-9956.

189 Y. Chen, Z.-L. Shi, L. Wei, B. Zhou, J. Tan, H.-L. Zhou and Y.-B. Zhang, J. Am. Chem. Soc., 2019, 141, 3298-3303.

190 W. Dai, F. Shao, J. Szczerbiński, R. McCaffrey, R. Zenobi, Y. Jin, A. D. Schlüter and W. Zhang, Angew. Chem., Int. Ed., 2016, 55, 213-217.

191 K. Dey, M. Pal, K. C. Rout, S. Kunjattu, A. Das, R. Mukherjee, U. K. Kharul and R. Banerjee, J. Am. Chem. Soc., 2017, 139, 13083-13091.

192 D. Zhou, X. Tan, H. Wu, L. Tian and M. Li, Angew. Chem., Int. Ed., 2019, 58, 1376-1381.

193 Q. Hao, C. Zhao, B. Sun, C. Lu, J. Liu, M. Liu, L.-J. Wan and D. Wang, J. Am. Chem. Soc., 2018, 140, 12152-12158.

194 L. Cao, H. Wu, Y. Cao, C. Fan, R. Zhao, X. He, P. Yang, B. Shi, X. You and Z. Jiang, Adv. Mater., 2020, 32, 2005565.

195 D. Rodríguez-San-Miguel, A. Yazdi, V. Guillerm, J. PérezCarvajal, V. Puntes, D. Maspoch and F. Zamora, Chem. Eur. J., 2017, 23, 8623-8627.

196 W. Kong, W. Jia, R. Wang, Y. Gong, C. Wang, P. Wu and J. Guo, Chem. Commun., 2019, 55, 75-78.

197 M. Zhang, L. Li, Q. Lin, M. Tang, Y. Wu and C. Ke, J. Am. Chem. Soc., 2019, 141, 5154-5158.

198 Y. Zhai, G. Liu, F. Jin, Y. Zhang, X. Gong, Z. Miao, J. Li, M. Zhang, Y. Cui, L. Zhang, Y. Liu, H. Zhang, Y. Zhao and Y. Zeng, Angew. Chem., Int. Ed., 2019, 58, 17679-17683.

199 H.-L. Qian, Y. Li and X.-P. Yan, J. Mater. Chem. A, 2018, 6, 17307-17311.

200 G. Zhang, M. Tsujimoto, D. Packwood, N. T. Duong, Y. Nishiyama, K. Kadota, S. Kitagawa and S. Horike, J. Am. Chem. Soc., 2018, 140, 2602-2609.

201 M. C. Daugherty, E. Vitaku, R. L. Li, A. M. Evans, A. D. Chavez and W. R. Dichtel, Chem. Commun., 2019, 55, 2680-2683.
202 A. Halder, S. Kandambeth, B. P. Biswal, G. Kaur, N. C. Roy, M. Addicoat, J. K. Salunke, S. Banerjee, K. Vanka, T. Heine, S. Verma and R. Banerjee, Angew. Chem., Int. Ed., 2016, 55, 7806-7810.

203 V. S. Vyas, F. Haase, L. Stegbauer, G. Savasci, F. Podjaski, C. Ochsenfeld and B. V. Lotsch, Nat. Commun., 2015, 6, 8508.

204 F. Haase, K. Gottschling, L. Stegbauer, L. S. Germann, R. Gutzler, V. Duppel, V. S. Vyas, K. Kern, R. E. Dinnebier and B. V. Lotsch, Mater. Chem. Front., 2017, 1, 1354-1361.

205 D. A. Pyles, W. H. Coldren, G. Eder, C. M. Hadad and P. L. McGrier, Chem. Sci., 2018, 9, 6417-6423.

206 J. Dong, Y. Wang, G. Liu, Y. Cheng and D. Zhao, CrystEngComm, 2017, 19, 4899-4904.

207 S. Ghosh, A. Nakada, M. A. Springer, T. Kawaguchi, K. Suzuki, H. Kaji, I. Baburin, A. Kuc, T. Heine, H. Suzuki, R. Abe and S. Seki, J. Am. Chem. Soc., 2020, 142, 9752-9762.

208 G. H. V. Bertrand, V. K. Michaelis, T.-C. Ong, R. G. Griffin and M. Dinca, Proc. Natl. Acad. Sci. U. S. A., 2013, 110, 4923-4928.

209 X. Wang, L. Chen, S. Y. Chong, M. A. Little, Y. Wu, W.-H. Zhu, R. Clowes, Y. Yan, M. A. Zwijnenburg, R. S. Sprick and A. I. Cooper, Nat. Chem., 2018, 10, 1180-1189.

210 H. Xu, J. Gao and D. Jiang, Nat. Chem., 2015, 7, 905-912.

211 X. Chen, M. Addicoat, E. Jin, L. Zhai, H. Xu, N. Huang, Z. Guo, L. Liu, S. Irle and D. Jiang, J. Am. Chem. Soc., 2015, 137, 3241-3247.

212 S. Kandambeth, V. Venkatesh, D. B. Shinde, S. Kumari, A. Halder, S. Verma and R. Banerjee, Nat. Commun., 2015, 6, 6786.

213 S. B. Alahakoon, K. Tan, H. Pandey, S. D. Diwakara, G. T. McCandless, D. I. Grinffiel, A. Durand-Silva, T. Thonhauser and R. A. Smaldone, J. Am. Chem. Soc., 2020, 142, 12987-12994.

214 C. S. Diercks and O. M. Yaghi, Science, 2017, 355, 8.

215 X. Chen, M. Addicoat, S. Irle, A. Nagai and D. Jiang, J. Am. Chem. Soc., 2013, 135, 546-549.

216 S. B. Alahakoon, G. Occhialini, G. T. McCandless, A. A. K. Karunathilake, S. O. Nielsen and R. A. Smaldone, CrystEngComm, 2017, 19, 4882-4885.

217 W. A. Braunecker, K. E. Hurst, K. G. Ray, Z. R. Owczarczyk, M. B. Martinez, N. Leick, A. Keuhlen, A. Sellinger and J. C. Johnson, Cryst. Growth Des., 2018, 18, 4160-4166.

218 F. Auras, L. Ascherl, A. H. Hakimioun, J. T. Margraf, F. C. Hanusch, S. Reuter, D. Bessinger, M. Döblinger, C. Hettstedt, K. Karaghiosoff, S. Herbert, P. Knochel, T. Clark and T. Bein, J. Am. Chem. Soc., 2016, 138, 16703-16710.

219 L. Ascherl, T. Sick, J. T. Margraf, S. H. Lapidus, M. Calik, C. Hettstedt, K. Karaghiosoff, M. Döblinger, T. Clark, K. W. Chapman, F. Auras and T. Bein, Nat. Chem., 2016, 8, 310-316.

220 N. Keller, T. Sick, N. N. Bach, A. Koszalkowski, J. M. Rotter, D. D. Medina and T. Bein, Nanoscale, 2019, 11, 23338-23345. 
221 L. M. Salonen, D. D. Medina, E. Carbó-Argibay, M. G. Goesten, L. Mafra, N. Guldris, J. M. Rotter, D. G. Stroppa and C. Rodríguez-Abreu, Chem. Commun., 2016, 52, 7986-7989.

222 Y.-L. Zhu, C.-L. Fu, Z.-W. Li and Z.-Y. Sun, J. Phys. Chem. Lett., 2020, 11, 179-183.

223 Y. Li, Q. Chen, T. Xu, Z. Xie, J. Liu, X. Yu, S. Ma, T. Qin and L. Chen, J. Am. Chem. Soc., 2019, 141, 13822-13828.

224 W. Hao, D. Chen, Y. Li, Z. Yang, G. Xing, J. Li and L. Chen, Chem. Mater., 2019, 31, 8100-8105.

225 X. Yan, H. Liu, Y. Li, W. Chen, T. Zhang, Z. Zhao, G. Xing and L. Chen, Macromolecules, 2019, 52, 7977-7983.

226 N. L. Campbell, R. Clowes, L. K. Ritchie and A. I. Cooper, Chem. Mater., 2009, 21, 204-206.

227 L. K. Ritchie, A. Trewin, A. Reguera-Galan, T. Hasell and A. I. Cooper, Microporous Mesoporous Mater., 2010, 132, 132-136.

228 J. Martín-Illán, D. Rodríguez-San-Miguel, C. Franco, I. Imaz, D. Maspoch, J. Puigmartí-Luis and F. Zamora, Chem. Commun., 2020, 56, 6704-6707.

229 H. Wei, S. Chai, N. Hu, Z. Yang, L. Wei and L. Wang, Chem. Commun., 2015, 51, 12178-12181.

230 G.-Y. Lee, J. Lee, H. T. Vo, S. Kim, H. Lee and T. Park, Sci. Rep., 2017, 7, 557.

231 B. P. Biswal, S. Chandra, S. Kandambeth, B. Lukose, T. Heine and R. Banerjee, J. Am. Chem. Soc., 2013, 135, 5328-5331.

232 G. Das, D. Balaji Shinde, S. Kandambeth, B. P. Biswal and R. Banerjee, Chem. Commun., 2014, 50, 12615-12618.

233 D. B. Shinde, H. B. Aiyappa, M. Bhadra, B. P. Biswal, P. Wadge, S. Kandambeth, B. Garai, T. Kundu, S. Kurungot and R. Banerjee, J. Mater. Chem. A, 2016, 4, 2682-2690.

234 Y. Peng, G. Xu, Z. Hu, Y. Cheng, C. Chi, D. Yuan, H. Cheng and D. Zhao, ACS Appl. Mater. Interfaces, 2016, 8, 18505-18512.

235 D. D. Medina, J. M. Rotter, Y. Hu, M. Dogru, V. Werner, F. Auras, J. T. Markiewicz, P. Knochel and T. Bein, J. Am. Chem. Soc., 2015, 137, 1016-1019.

236 J. W. Colson, A. R. Woll, A. Mukherjee, M. P. Levendorf, E. L. Spitler, V. B. Shields, M. G. Spencer, J. Park and W. R. Dichtel, Science, 2011, 332, 228-231.

237 F. Haase and B. V. Lotsch, Chem. Soc. Rev., 2020, 49, 8469-8500.
238 P.-L. Wang, S.-Y. Ding, Z.-C. Zhang, Z.-P. Wang and W. Wang, J. Am. Chem. Soc., 2019, 141, 18004-18008.

239 S. J. Lyle, T. M. Osborn Popp, P. J. Waller, X. Pei, J. A. Reimer and O. M. Yaghi, J. Am. Chem. Soc., 2019, 141, 11253-11258.

240 X. Li, C. Zhang, S. Cai, X. Lei, V. Altoe, F. Hong, J. J. Urban, J. Ciston, E. M. Chan and Y. Liu, et al., Nat. Commun., 2018, 9, 2998.

241 F. Haase, E. Troschke, G. Savasci, T. Banerjee, V. Duppel, S. Dörfler, M. M. J. Grundei, A. M. Burow, C. Ochsenfeld and S. Kaskel, et al., Nat. Commun., 2018, 9, 2600.

242 K. Wang, Z. Jia, Y. Bai, X. Wang, S. E. Hodgkiss, L. Chen, S. Y. Chong, X. Wang, H. Yang, Y. Xu, F. Feng, J. W. Ward and A. I. Cooper, J. Am. Chem. Soc., 2020, 142, 11131-11138.

243 J. L. Segura, S. Royuela and M. Mar Ramos, Chem. Soc. Rev., 2019, 48, 3903-3945.

244 E. Jin, M. Asada, Q. Xu, S. Dalapati, M. A. Addicoat, M. A. Brady, H. Xu, T. Nakamura, T. Heine, Q. Chen and D. Jiang, Science, 2017, 357, 673-676.

245 S. Bi, C. Yang, W. Zhang, J. Xu, L. Liu, D. Wu, X. Wang, Y. Han, Q. Liang and F. Zhang, Nat. Commun., 2019, 10, 2467.

246 A. Acharjya, L. Longworth-Dunbar, J. Roeser, P. Pachfule and A. Thomas, J. Am. Chem. Soc., 2020, 142, 14033-14038.

247 S. Thomas, H. Li, R. R. Dasari, A. M. Evans, I. Castano, T. G. Allen, O. G. Reid, G. Rumbles, W. R. Dichtel, N. C. Gianneschi, S. R. Marder, V. Coropceanu and J.-L. Brédas, Mater. Horiz., 2019, 6, 1868-1876.

248 W. Liu, X. Luo, Y. Bao, Y. P. Liu, G.-H. Ning, I. Abdelwahab, L. Li, C. T. Nai, Z. G. Hu and D. Zhao, et al., Nat. Chem., 2017, 9, 563-570.

249 W. Liu, M. Ulaganathan, I. Abdelwahab, X. Luo, Z. Chen, S. J. Rong Tan, X. Wang, Y. Liu, D. Geng and Y. Bao, et al., ACS Nano, 2018, 12, 852-860.

250 D. L. Pastoetter, S. Xu, M. Borrelli, M. Addicoat, B. P. Biswal, S. Paasch, A. Dianat, H. Thomas, R. Berger, S. Reineke, E. Brunner, G. Cuniberti, M. Richter and X. Feng, Angew. Chem., Int. Ed., 2020, 59, 23620-23625. 